\title{
Cohomology of generalized supergrassmannians and character formulae for basic classical Lie superalgebras
}

\author{
Caroline Gruson 7 and Vera Serganova 2
}

\section{INTRODUCTION}

The famous Borel-Weil-Bott theorem states that, for a reductive complex algebraic group $G$, the cohomology groups of any invertible sheaf on the flag variety $G / B$ are non zero in at most one degree, and in this degree the cohomology group is a simple $G$-module. This statement is no longer true for Chevalley groups in characteristic $p$, the cohomology group is non-zero only in one degree (for a dominant weight) but it is not a simple $G$ module anymore. Its structure is rather complicated and interesting, and studying it leads to Kazhdan-Lusztig theory.

Let now $G$ be a complex basic classical supergroup. Studying the cohomology groups on the flag supervariety $G / B$ was initiated by Penkov and others ([20, [23]). They discovered that the Borel-Weil-Bott theorem is not always true in this case and proved that it is true for so called typical invertible sheaves.

This question is closely related to the representation theory of the corresponding basic classical Lie superalgebra $\mathfrak{g}$. The category of finite-dimensional $\mathfrak{g}$-modules is not semisimple, only typical simple modules do not have extensions with other simple modules. The problem of computing characters of atypical simple finite dimensional $\mathfrak{g}$-modules is a full-time occupation for several persons since 1977. The formula for typical highest weight modules was found in [17]. In 1980 Bernstein and Leites found the character formula in case $\mathfrak{g}=\mathfrak{s l}(1, n)([2])$, later this formula was generalised for $\mathfrak{o} \mathfrak{s}(2,2 n)$ and for the so called singly atypical modules and generic atypical modules (see [29] and [30]). Similar type formulae were conjectured in [18] for affine superalgebras. In 1996, the second author [24] solved the problem completely for $\mathfrak{g l}(m, n)$ using the geometry of flag supervarieties. Later Brundan [3] solved this problem by a different, purely algebraic, method and discovered a remarkable connection with the representation theory of $\mathfrak{g l}(\infty)$. Brundan's method was developed further in [8], 4], [5], [6], [7].

In this paper we generalise the method of 24 ] to the orthosymplectic groups $O S P(m, 2 n)$. The results were announced in [25] without proof and one statement (typical lemma) was formulated with a mistake there. We take a different Borel subgroup in this paper and use the language of weight diagrams (invented by Brundan and Stroppel for $G L(m, n)$ ). This makes the combinatorics much easier and allows one to formulate a precise algorithm for the computation of the characters of all simple modules. In the $\mathfrak{g l}$ case, there exist finite dimensional analogues of Verma modules, called Kac modules, which can be interpreted as cohomology groups of bundles over the flag supermanifold, and which also have a nice

\footnotetext{
${ }^{1}$ U.M.R. 7502 du CNRS, Institut Elie Cartan, Universite Henri Poincaré (Nancy 1), BP 239, 54506 Vandoeuvre-les-Nancy Cedex, France. E-mail: Caroline.Gruson@iecn.u-nancy.fr

${ }^{2}$ Department of Mathematics, University of California, Berkeley, CA,94720-3840 USA. E-mail: serganov@Math.Berkeley.EDU
} 
algebraic behavior. Unfortunately (?), there is no such analogue in the orthosymplectic case, so we use the geometrical method.

Let us explain the main idea of the method. As was pointed out by Penkov, one can easily write down the character of the Euler characteristic for any invertible sheaf on a flag supervariety using classical Borel-Weil-Bott theory. Thus, if one knows the multiplicities of simple modules in cohomology groups, one hopes to express the characters of simple modules as linear combinations of characters of Euler characteristic of some invertible sheaves. We manage to find these multiplicities for some generalized super grassmannians $G / P$ for certain parabolic subgroups $P$, and use this information to compute characters.

There are several complications in the case of $O S P(m, 2 n)$ compared with $G L(m, n)$. First, in many cases the Euler characteristic is zero on $G / B$, and one should consider instead $G / Q$ for a suitable choice of a parabolic subgroup $Q$ which depends on the module whose character we want to calculate. Next, there is no distinguished Borel subgroup in $O S P(m, 2 n)$, hence there are non-vanishing cohomology groups in many degrees. Finally, the recursion process involving the computation of the cohomology is more complicated due to the existence of the so-called exceptional pairs (see Section 10 in the paper for details). On the other hand, there is one advantage: the character of a simple $\operatorname{OSP}(m, 2 n)$ -module is always a finite linear combination of Euler characteristics, in contrast with the $G L(m, n)$ case where the combination is always infinite.

The organisation of the paper is as follows. In Section 2 we fix the notations. Section 3 contains general statements about cohomology groups of vector bundles on a supergrassmannian $G / P$. In Section 4 we compute the character of the Euler characteristic, and, for parabolic subgroups $P \subset Q \subset G$, relate the cohomology on $G / P$ with those on $G / Q$ and $Q / P$, using Leray spectral sequence. Section 5 contains a complete classification of the blocks in the category of finite-dimensional $\mathfrak{g}$-modules (Theorem 2) and introduces translation functors. In Section 6, we introduce weight diagrams and translate the results of Section 5 into this language. In Section 7 we explain how to reduce the computation of the cohomology to the case of the most atypical blocks of $\mathfrak{g l}(k, k), \mathfrak{o s p}(2 k+1,2 k), \mathfrak{o s p}(2 k, 2 k)$ and $\mathfrak{o s p}(2 k+2,2 k)$. (Afterwards we concentrate on the orthosymplectic case, since the general linear case is done in [24]). This last computation is done recursively in Sections 8,9 and 10 starting with $\mathfrak{o s p}(2 k+1,2 k)$ (resp. osp $(2 k, 2 k), \mathfrak{o s p}(2 k+2,2 k)$ ) and getting down to $\mathfrak{o s p}(2 k-1,2 k-2)$ (resp. $\mathfrak{o s p}(2 k-2,2 k-2), \mathfrak{o s p}(2 k, 2 k-2))$. In Section 11, the recursion is solved. We construct a graph whose vertices are weight diagrams and describe a combinatorial algorithm for the computation of cohomology groups, in terms of paths in this graph (Proposition 7 and Theorem 3). Section 12 presents an algorithm for calculating characters (Theorem 4) and contains some examples. In Section 13, we present a simplfication of the Section 11's algorithm. In the general linear case, this simplification leads to the equivalence (proven in [22]) of algorithms appearing in [24] and [3].

Both authors thank Laurent Gruson for hospitality and stimulating discussions. The second author would like to thank Ian Musson for explaining the method of weight diagrams and fruitful discussions on supergeometry.

\section{Notations}

Let $\mathfrak{g}=\mathfrak{g}_{0} \oplus \mathfrak{g}_{1}$ be a basic classical complex Lie superalgebra, i.e. $\mathfrak{g}=\mathfrak{g l}(m, n)$ or $\mathfrak{o} \mathfrak{s p}(m, n)$. By $G$ we denote the linear algebraic groups $G L(m, n)$ and $O S P(m, n)$ respectively [19]. For a Lie subalgebra (e.g. $\mathfrak{a} \subset \mathfrak{g}$ ), we denote with the capital Latin letter 
(in this case $A \subset G$ ) the connected complex algebraic supergroup with the given Lie superalgebra. For all the superalgebras considered in this paper such supergroups are well defined.

We fix a Cartan subalgebra $\mathfrak{h}$ of $\mathfrak{g}$. Denote by $\Delta$ the set of roots of $\mathfrak{g}$ with respect to $\mathfrak{h}$. Let $\mathfrak{b}$ be a Borel subalgebra of $\mathfrak{g}$ containing $\mathfrak{h}$, it defines a set of positive roots $\Delta^{+}$, and we define $\rho=\rho_{0}-\rho_{1}$, where $\rho_{0}$ is the half sum of the positive even roots and $\rho_{1}$ is the half sum of positive odd roots. Recall that since $\mathfrak{g}$ is basic classical, it is equipped with a non degenerate invariant bilinear form and the restriction of this bilinear form to $\mathfrak{h}$ is also non degenerate. We will denote this form by $($,$) . We denote by W$ the Weyl group of the even part $\mathfrak{g}_{0}$. Recall that in our case the Cartan subalgebra $\mathfrak{h}$ of $\mathfrak{g}$ is also a Cartan subalgebra of $\mathfrak{g}_{0}$. For a Lie subalgebra $\mathfrak{a} \subset \mathfrak{g}$ such that $\mathfrak{h} \subset \mathfrak{a}$ we denote by $\Delta(\mathfrak{a}) \subset \Delta$ the set of roots of $\mathfrak{a}$.

A weight $\lambda \in \mathfrak{h}^{*}$ is integral if it induces a one-dimensional representation of the Cartan subgroup $H$. In the paper we consider only integral weights. Thus, by a weight we always mean an integral weight. Moreover, we only consider modules which are integrable with respect to the group $G$ : all those have integral weights. Define the standard order on the set of integral weights: $\lambda \leq \mu$ iff $\mu-\lambda=\sum_{\alpha \in \Delta^{+}} n_{\alpha} \alpha$ where all $n_{\alpha}$ are non-negative integers.

For any integral weight $\lambda$, we will denote by $L_{\lambda}$ the simple $\mathfrak{g}$-module with highest weight $\lambda$, and if $\mathfrak{a}$ is a Lie subalgebra of $\mathfrak{g}$ for which it makes sense, we will denote by $L_{\lambda}(\mathfrak{a})$ the irreducible $\mathfrak{a}$-module with highest weight $\lambda$. Recall that $\lambda$ is called dominant (resp. $\mathfrak{a}$ dominant) if $L_{\lambda}$ (resp. $\left.L_{\lambda}(\mathfrak{a})\right)$ is finite-dimensional (in this case $L_{\lambda}$ (resp. $L_{\lambda}(\mathfrak{a})$ ) has a natural structure of $G$-module (resp. $A$-module)).

Let $\mathcal{U}(\mathfrak{g})$ be the universal enveloping algebra of $\mathfrak{g}$ and $\mathcal{Z}(\mathfrak{g})$ be its center. For every weight $\lambda$, we write $\chi_{\lambda}$ for the corresponding central character. A central character $\chi$ is dominant if there exists a dominant $\lambda$ such that $\chi=\chi_{\lambda}$.

Finally, let us recall the description of $\Delta$ (see [16]). Let $\mathfrak{g}=\mathfrak{g l}(m, n), \mathfrak{o s p}(2 m, 2 n)$ or $\mathfrak{o s p}(2 m+1,2 n)$. Then $\operatorname{dim} \mathfrak{h}=m+n$ and one can choose a basis $\varepsilon_{1}, \ldots, \varepsilon_{m}, \delta_{1}, \ldots, \delta_{n}$ of $\mathfrak{h}^{*}$ such that

$$
\left(\varepsilon_{i}, \varepsilon_{j}\right)=\delta_{i j},\left(\varepsilon_{i}, \delta_{j}\right)=0,\left(\delta_{i}, \delta_{j}\right)=-\delta_{i j}
$$

The even roots $\Delta_{0}$ of $\mathfrak{g l}(m, n)$ are all vectors of the form $\varepsilon_{i}-\varepsilon_{j}$ and $\delta_{i}-\delta_{j}$ with $i \neq j$. The odd roots $\Delta_{1}$ of $\mathfrak{g l}(m, n)$ are all vectors of the form $\varepsilon_{i}-\delta_{j}$ and $\delta_{i}-\varepsilon_{j}$.

The even roots $\Delta_{0}$ of $\mathfrak{o s p}(2 m, 2 n)$ are all vectors of the form $\pm \varepsilon_{i} \pm \varepsilon_{j}, \pm \delta_{i} \pm \delta_{j}$ (the signs can be chosen independently) with $i \neq j$ and $2 \delta_{i}$. The odd roots $\Delta_{1}$ of $\mathfrak{o s p}(2 m, 2 n)$ are all vectors of the form $\pm \varepsilon_{i} \pm \delta_{j}$.

The even roots $\Delta_{0}$ of $\mathfrak{o s p}(2 m+1,2 n)$ are all vectors of the form $\pm \varepsilon_{i} \pm \varepsilon_{j}, \pm \delta_{i} \pm \delta_{j}$ with $i \neq j, \pm \varepsilon_{i}$ and $\pm 2 \delta_{i}$. The odd roots $\Delta_{1}$ of $\mathfrak{o s p}(2 m+1,2 n)$ are all vectors of the form $\pm \varepsilon_{i} \pm \delta_{j}$ and $\pm \delta_{i}$.

We define a parity on the weight lattice by saying that $\varepsilon_{i}$ (resp. $\delta_{j}$ ) is even (resp. odd). Then the category of finite dimensional $G$-modules splits in a direct summand of two parts, one in which the weight spaces have the same parity as the corresponding weight, and one in which the parities differ. In this paper, we will only consider the first part.

\section{Geometric induction}

Let $\mathfrak{p}$ be any parabolic subalgebra of $\mathfrak{g}$ containing $\mathfrak{b}$ and $\mathfrak{l}$ denote the reductive part of $\mathfrak{p}$.

For a $P$-module e.g. $V$, we denote by the calligraphic letter $\mathcal{V}$ the vector bundle $G \times{ }_{P} V$ over the generalized grassmannian $G / P$. Note that the space of sections of $\mathcal{V}$ on any open 
set has a natural structure of $\mathfrak{g}$-module, in other words the sheaf of sections of $\mathcal{V}$ is a $\mathfrak{g}$-sheaf. Therefore the cohomology groups $\left(H^{i}(G / P, \mathcal{V})\right)$ are $\mathfrak{g}$-modules. For details see [19], 23], 20].

Define the functor $\Gamma_{i}$ from the category of $\mathfrak{p}$-modules to the category of $\mathfrak{g}$-modules by

$$
\Gamma_{i}(G / P, V):=\left(H^{i}\left(G / P, \mathcal{V}^{*}\right)\right)^{*} .
$$

Lemma 1. - The functors $\Gamma_{i}$ have the following properties:

1) if

$$
0 \rightarrow U \rightarrow V \rightarrow W \rightarrow 0
$$

is a short exact sequence of $P$-modules, then one has

$$
\ldots \rightarrow \Gamma_{1}(G / P, W) \rightarrow \Gamma_{0}(G / P, U) \rightarrow \Gamma_{0}(G / P, V) \rightarrow \Gamma_{0}(G / P, W) \rightarrow 0
$$

(long exact sequence).

2) if $M$ is a $\mathfrak{g}$-module and $V$ is a P-module, the following holds :

$$
\Gamma_{i}(G / P, V \otimes M)=\Gamma_{i}(G / P, V) \otimes M .
$$

The proof is an adaptation of standard arguments (see [15]).

Lemma 2. - The module $\Gamma_{0}(G / P, V)$ is the maximal finite-dimensional quotient of $\mathcal{U}(\mathfrak{g}) \otimes_{\mathcal{U}(\mathfrak{p})}$ $V$.

Proof. - Let $\tilde{V}$ be the maximal finite-dimensional quotient of $\mathcal{U}(\mathfrak{g}) \otimes_{\mathcal{U}(\mathfrak{p})} V$. By duality $\tilde{V}^{*}$ is the maximal finite-dimensional submodule in the coinduced module $\operatorname{Hom}_{\mathcal{U}(\mathfrak{p})}\left(\mathcal{U}(\mathfrak{g}), V^{*}\right)$.

Let $\Gamma\left(V^{*}\right):=H^{0}\left(G / P, \mathcal{V}^{*}\right)$. By definition

$$
\Gamma\left(V^{*}\right)=\left\{\gamma \in \mathbb{C}[G] \otimes V^{*} \mid \gamma(g p)=p^{-1} \gamma(g), g \in G, p \in P\right\} .
$$

Let $\pi$ denote the composition of the standard maps $V \rightarrow \mathcal{U}(\mathfrak{g}) \otimes_{\mathcal{U}(\mathfrak{p})} V \rightarrow \tilde{V}$ and $\pi^{*}: \tilde{V}^{*} \rightarrow V^{*}$ be the dual map. Then $\gamma_{v}(g)=\pi^{*}\left(g^{-1} v\right)$ is a vector in $\Gamma\left(V^{*}\right)$. Hence we can define a $G$-module homomorphism $\varphi: \tilde{V}^{*} \rightarrow \Gamma\left(V^{*}\right)$ by putting $\varphi(v):=\gamma_{v}$. We claim that $\varphi$ is injective. Indeed, assume $X=\operatorname{Ker} \varphi \neq 0$. But $\pi^{*}(X) \neq 0$ for every non-trivial $G$-submodule $X \subset \tilde{V}^{*}$. Therefore $X$ contains $v$ such that $\gamma_{v}(e) \neq 0$, hence $\varphi(X) \neq 0$. Contradiction.

On the other hand, the map ev $: \Gamma\left(V^{*}\right) \rightarrow V^{*}$ defined by $e v(\gamma):=\gamma(e)$ induces a homomorphism of $\mathfrak{g}$-modules $j: \Gamma\left(V^{*}\right) \rightarrow \operatorname{Hom}_{\mathcal{U}(\mathfrak{p})}\left(\mathcal{U}(\mathfrak{g}), V^{*}\right)$. Since $\Gamma\left(V^{*}\right)$ is finitedimensional $j\left(\Gamma\left(V^{*}\right)\right) \subset \tilde{V}^{*}$. Again we claim that $j$ is injective. Indeed, choose a nilpotent superalgebra $\mathfrak{m}^{-}$such that $\mathfrak{g}=\mathfrak{p} \oplus \mathfrak{m}^{-}$, and let $M^{-}$be the corresponding supergroup. It is known that (exactly as in classical case) $M^{-} P$ is dense in $G$ (see [21]). Hence $e v$ is injective on the subspace of invariants $\Gamma\left(V^{*}\right)^{\mathfrak{m}^{-}}$. Let $Y=\operatorname{Ker} j$. Then $Y^{m^{-}}=0$. That implies $Y=0$.

Thus, we have two injective morphisms $\varphi: \tilde{V}^{*} \rightarrow \Gamma\left(V^{*}\right)$ and $j: \Gamma\left(V^{*}\right) \rightarrow \tilde{V}^{*}$. Since the spaces are finite-dimensional, both $\varphi$ and $j$ are isomorphisms. Therefore, the dual modules $\tilde{V}$ and $\Gamma_{0}(G / P, V)$ are also isomorphic.

Definition 1. (Penkov's remark [23]) - Let $\left(X, \mathcal{O}_{X}\right)$ be a generalized grassmannian, and $\left(X_{0}, \mathcal{O}_{X_{0}}\right)$ be the underlying algebraic variety, $\mathcal{V}$ be a $G$-vector bundle on $X$. Then the corresponding sheaf $\tilde{\mathcal{V}}$ on $X_{0}$ has a filtration by $\mathcal{O}_{X_{0}}$-modules such that the associated graded module (in degree $i$ ) is isomorphic to the $G_{0}$-bundle $\mathcal{V}_{X_{0}} \otimes S^{i}\left(\mathcal{N}_{X_{0}}^{*} X\right)=: \tilde{\mathcal{V}}^{i}$. The whole module will be denoted by $\mathcal{I}_{X_{0}}\left(\mathcal{V} \otimes S^{\bullet}\left(\mathcal{N}_{X_{0}}^{*} X\right)\right)$. 
By definition $H^{k}(X, \mathcal{V})=H^{k}\left(X_{0}, \tilde{\mathcal{V}}\right)$. We have, if we denote by $C h(V)$ the character of a $\mathfrak{g}$ module $V$,

$$
C h\left(H^{k}\left(X_{0}, G r(\tilde{\mathcal{V}})\right)\right) \geq C h\left(H^{k}(X, \mathcal{V})\right)
$$

as can be seen with the long exact sequence coming with the filtration of $\tilde{\mathcal{V}}$. The $\geq$ means that each $\mathfrak{g}_{0}$-module occurring in $H^{k}(X, \mathcal{V})$ appears in $H^{k}\left(X_{0}, G r(\tilde{\mathcal{V}})\right)$ with at least the same multiplicity. Note that the inequality becomes an equality when computing the Euler characteristic.

Lemma 3. - If $L_{\mu}$ occurs in $\Gamma_{i}\left(G / P, L_{\lambda}(\mathfrak{p})\right)$ with non-zero multiplicity, then $\mu+\rho=$ $w(\lambda+\rho)-\sum_{\alpha \in I} \alpha$ for some $w \in W$ of length $i$ and $I \subset \Delta_{1}^{+}$.

Proof. - We make use of Penkov's remark. First, note that

$$
\operatorname{Gr}\left(S^{i}\left(\mathcal{N}_{X_{0}} X\right)\right)=G_{0} \times_{P_{0}} S^{i}\left(\mathfrak{g} /\left(\mathfrak{g}_{0} \oplus \mathfrak{p}_{1}\right)\right)
$$

which is a $G_{0}$-bundle on $G_{0} / P_{0}$, so:

$$
C h\left(H^{k}\left(G / P, \mathcal{L}_{\lambda}(\mathfrak{p})^{*}\right)\right) \leq C h\left(H^{k}\left(G_{0} / P_{0}, \mathcal{I}_{G_{0} / P_{0}} \mathcal{L}_{\lambda}^{*}(\mathfrak{p}) \otimes S^{\bullet}\left(\mathfrak{g} /\left(\mathfrak{g}_{0} \oplus \mathfrak{p}_{1}\right)\right)^{*}\right)\right) .
$$

Assume $L_{\mu}$ occurs in $\Gamma_{i}\left(G / P, L_{\lambda}^{*}(\mathfrak{p})\right)$. It means that $L_{\mu}^{*}$ occurs in $H^{i}\left(G / P, \mathcal{I}_{G_{0} / P_{0}} \mathcal{L}_{\lambda}^{*}(\mathfrak{p})\right)$. Therefore $L_{\mu}\left(\mathfrak{g}_{0}\right)^{*}$ occurs in $H^{i}\left(G_{0} / P_{0}, \mathcal{L}_{\lambda}^{*}(\mathfrak{p}) \otimes S^{\bullet}\left(\mathfrak{g} /\left(\mathfrak{g}_{0} \oplus \mathfrak{p}_{1}\right)\right)^{*}\right)$. The latter sheaf has a filtration by $G_{0}$-bundles whose simple quotients are of the form $\mathcal{L}_{\lambda-\sum_{\gamma \in J \subset \Delta_{1}^{+}}^{*}}\left(\mathfrak{p}_{0}\right)$. By the usual (non-graded) Borel-Weil-Bott theorem, if $w\left(\nu+\rho_{0}\right)-\rho_{0}$ is regular and $\mathfrak{g}_{0}$-dominant, then

Therefore

$$
H^{i}\left(G_{0} / P_{0}, \mathcal{L}_{\nu}\left(\mathfrak{p}_{0}\right)^{*}\right)=\left\{\begin{array}{c}
L_{w\left(\nu+\rho_{0}\right)-\rho_{0}}\left(\mathfrak{g}_{0}\right)^{*} \text { if } l(w)=i \\
0 \text { otherwise }
\end{array}\right.
$$

$$
\mu=w\left(\lambda-\sum_{\gamma \in J \subset \Delta_{1}^{+}} \gamma+\rho_{0}\right)-\rho_{0} .
$$

Using $\rho_{0}=\rho+\rho_{1}$, we obtain

$$
\mu=w(\lambda+\rho)-\rho-\sum_{\alpha \in I \subset \Delta_{1}^{+}} \alpha,
$$

where $I=\left(w(J) \cap \Delta_{1}^{+}\right) \cup-\left(w\left(\Delta_{1}^{+}-J\right) \cap \Delta_{1}^{-}\right)$.

Write $\mathfrak{p}=\mathfrak{l} \oplus \mathfrak{m}, \mathfrak{g}=\mathfrak{m}^{-} \oplus \mathfrak{l} \oplus \mathfrak{m}$, where $\mathfrak{m}$ is the nilpotent part of $\mathfrak{p}$. Consider the projection

$$
\phi: \mathcal{U}(\mathfrak{g})=\mathcal{U}\left(\mathfrak{m}^{-}\right) \mathcal{U}(\mathfrak{l}) \mathcal{U}(\mathfrak{m}) \rightarrow \mathcal{U}\left(\mathfrak{m}^{-}\right) \mathcal{U}(\mathfrak{l})
$$

with the kernel $\mathcal{U}(\mathfrak{g}) \mathfrak{m}$. The restriction of $\phi$ to $\mathcal{Z}(\mathfrak{g})$ induces the injective homomorphism of centers $\mathcal{Z}(\mathfrak{g}) \rightarrow \mathcal{Z}(\mathfrak{l})$. We denote by $\Phi$ the dual map

$$
\operatorname{Hom}(\mathcal{Z}(\mathfrak{l}), \mathbb{C}) \rightarrow \operatorname{Hom}(\mathcal{Z}(\mathfrak{g}), \mathbb{C}) .
$$

Lemma 4. - If $V$ is an irreducible $\mathfrak{p}$-module admitting a central character $\chi$, then the $\mathfrak{g}$-module $\Gamma_{i}(G / P, V)$ admits the central character $\Phi(\chi)$.

Proof. - Consider another projection

$$
\psi: \mathcal{U}(\mathfrak{g})=\mathcal{U}(\mathfrak{m}) \otimes \mathcal{U}(\mathfrak{l}) \otimes \mathcal{U}\left(\mathfrak{m}^{-}\right) \rightarrow \mathcal{U}(\mathfrak{l}) \mathcal{U}\left(\mathfrak{m}^{-}\right)
$$

with the kernel $\mathfrak{m} \mathcal{U}(\mathfrak{g})$, and the corresponding dual map

$$
\Psi: \operatorname{Hom}(\mathcal{Z}(\mathfrak{l}), \mathbb{C}) \rightarrow \operatorname{Hom}(\mathcal{Z}(\mathfrak{g}), \mathbb{C}) .
$$


Note that any element $x \in \mathcal{U}(\mathfrak{g})$ acts on the set of sections of the vector bundle $\mathcal{V}^{*}$ over any open subset $U \subset G / P$. We denote this action by $L_{x}$.

We claim that if $\gamma$ is a section of $\mathcal{V}^{*}$ over any open subset $U \subset G / P$ and $z \in \mathcal{Z}(\mathfrak{g})$ belongs to the kernel of $\psi$, then $L_{z}(\gamma)=0$. Since $z$ commutes with the group action, it suffices to prove that $L_{z}(\gamma) \in I_{P}$, where $I_{P}$ stands for the subspace of sections which are zero at $P$. Our claim now follows immediately from the facts that, if $x \in \mathfrak{m}$, then $L_{x}(\gamma) \in I_{P}$, and if $x \in \mathfrak{l}$, then the value of $L_{x}(\gamma)$ at $p \in P$ equals $x(\gamma(p))$. Thus, if $\zeta$ is the central character of $\mathfrak{l}$-module $V^{*}$, then the space of sections $\Gamma\left(U, \mathcal{V}^{*}\right)$ (as a $\mathfrak{g}$-module) admits central character $\Psi(\zeta)$.

Using the Chech complex for the computation of the cohomology groups of $\mathcal{V}^{*}$, one can show easily that the cohomology groups $H^{i}\left(G / P, \mathcal{V}^{*}\right)$ also admit central character $\Psi(\zeta)$. Going to the dual modules provides the statement.

The following corollary of Lemma 1(2) and Lemma 4 will be used a lot in this paper.

Corollary 1. - For any finite-dimensional $\mathfrak{g}$-module $M$ (resp. finite-dimensional $\mathfrak{p}$-module $V)$ let $M^{\chi}\left(r e s p . V^{\Phi^{-1}(\chi)}\right)$ denote the component with generalized central character $\chi$ (resp. with generalized central character lying in $\left.\Phi^{-1}(\chi)\right)$. Then

$$
\Gamma_{i}\left(G / P,(V \otimes M)^{\Phi^{-1}(\chi)}\right)=\left(\Gamma_{i}(G / P, V) \otimes M\right)^{\chi} .
$$

Let $\mathcal{F}$ be the category of finite dimensional $\mathfrak{g}$-modules semisimple over $\mathfrak{h}$; this category decomposes into blocks $\mathcal{F} \chi$, where $\mathcal{F}^{\chi}$ consists of all finite dimensional modules with (generalized) central character $\chi$.

Remark - Let $\mathfrak{l}$ be the Levi subalgebra of $\mathfrak{p}$. If $V$ is a module belonging to the block $\mathcal{F}^{\tau}(\mathfrak{l})$ consisting all finite dimensional modules with (generalized) central character $\tau$, then $\Gamma_{i}(G / P, V)$ belongs to the block $\mathcal{F}^{\Phi(\tau)}$. That provides a correspondence between blocks of $\mathfrak{l}$ and blocks of $\mathfrak{g}$.

Definition 2. - Let $\lambda$ be a $\mathfrak{g}$-dominant weight. Define $A(\lambda)$ to be a maximal possible set of mutually orthogonal positive isotropic roots $\alpha_{i}$ of $\mathfrak{g}$ such that $\left(\lambda+\rho, \alpha_{i}\right)=0, A(\lambda)=$ $\left\{\alpha_{1}, \ldots \alpha_{l}\right\}$. We put $\# A(\lambda)=\# \lambda$, and call it the degree of atypicality of $\lambda$ (say it is 0 if $A(\lambda)=\emptyset$ : then $\lambda$ is called typical).

Although the choice of $A(\lambda)$ is not unique, the degree of atypicality does not depend on it.

Then, for any weight $\mu, \chi_{\lambda}=\chi_{\mu}$ is equivalent to the fact that $\mu$ can be written as $w\left(\lambda+\rho+n_{1} \alpha_{1}+\ldots+n_{l} \alpha_{l}\right)-\rho$, where $w \in W$ and $n_{i} \in \mathbb{C}$ for all $i=1, \ldots, l$ (see [26]).

Notice that if $\chi_{\lambda}=\chi_{\mu}$, then $\lambda$ and $\mu$ have the same degree of atypicality. So the degree of atypicality is a well defined notion for a central character.

For any non-isotropic $\beta \in \Delta$ put $\check{\beta}:=\frac{2 \beta}{(\beta, \beta)}$.

Definition 3. - A parabolic subalgebra $\mathfrak{p} \subset \mathfrak{g}$ with Levi part $\mathfrak{l}$ is called admissible for a central character $\chi$ if, for any dominant $\lambda$ such that $\chi_{\lambda}=\chi$, one has $(\lambda+\rho, \check{\beta}) \geq 0$ for all $\beta \in \Delta_{0}^{+}-\Delta(\mathfrak{l})$.

For example, if $\mathfrak{g}=\mathfrak{g l}(m, n)$, then a distinguished Borel subalgebra ([16]) is admissible for any central character, and therefore so is any parabolic subalgebra containing this distinguished Borel subalgebra. If the simple roots of a Borel subalgebra are all isotropic, then $\rho=0$ and any parabolic subalgebra containing this Borel subalgebra is admissible for any central character. 
Lemma 5. (Typical lemma) - Let $\lambda$ be a dominant weight, let $\mathfrak{p}$ be a parabolic subalgebra of $\mathfrak{g}$ admissible for $\chi_{\lambda}$. Assume $A(\lambda) \subset \Delta(\mathfrak{l})$ and $(\lambda+\rho, \check{\beta})>0$ for all $\beta \in \Delta_{0}^{+}-\Delta(\mathfrak{l})$ (in this case we will call $\lambda \mathfrak{p}$-typical). Then,

$$
\Gamma_{i}\left(G / P, L_{\lambda}(\mathfrak{p})\right)=\left\{\begin{array}{c}
0 \text { if } i>0 \\
L_{\lambda}(\mathfrak{g}) \text { if } i=0
\end{array}\right.
$$

Proof. - Let $L_{\mu}$ be an irreducible subquotient in $\Gamma_{i}\left(G / P, L_{\lambda}(\mathfrak{p})\right)$. Then by Lemma 3 there exist $w \in W$ and $I \subset \Delta_{1}^{+}$such that

$$
\mu=w(\lambda+\rho)-\rho-\sum_{\alpha \in I} \alpha .
$$

Choose an element $h \in \mathfrak{h}^{*}$ such that

$$
\Delta(\mathfrak{p})=\{\alpha \in \Delta \mid(\alpha, h) \geq 0\} .
$$

Note that $\Delta(\mathfrak{l})=\{\alpha \in \Delta \mid(\alpha, h)=0\}$.

We claim that $(\mu, h) \leq(\lambda, h)$. Indeed, let $s \in W$ be such that $s(\lambda+\rho)$ belongs to the positive Weyl chamber. Since $(\lambda+\rho, \check{\beta}) \geq 0$ for all $\beta \in \Delta_{0}^{+}-\Delta(\mathfrak{l}), s$ belongs to the Weyl group of $\mathfrak{l}_{0}$. Since $s(h)=h$ we have $(s(\lambda+\rho), h)=(\lambda+\rho, h)$. Then

$$
w(\lambda+\rho)=s(\lambda+\rho)-\sum_{\beta \in \Delta_{0}^{+}} k_{\beta} \beta .
$$

with some non-negative $k_{\beta}$ since $s(\lambda+\rho)$ lies in the positive Weyl chamber. Therefore

$$
(w(\lambda+\rho), h)=(s(\lambda+\rho), h)-\sum_{\beta \in \Delta_{0}^{+}} k_{\beta}(\beta, h) \leq(s(\lambda+\rho), h)=(\lambda+\rho, h) .
$$

Therefore by (1)

$$
(\mu+\rho, h)=(w(\lambda+\rho), h)-\sum_{\alpha \in I}(\alpha, h) \leq(w(\lambda+\rho), h) \leq(\lambda+\rho, h) .
$$

On the other hand, $\chi_{\lambda}=\chi_{\mu}$ implies

$$
u(\mu+\rho)=\lambda+\rho+\sum_{\alpha \in A(\lambda)} k_{\alpha} \alpha
$$

for some $u \in W$. Since $\mu$ is dominant, we obtain by the same argument as above

$$
(\mu+\rho, h) \geq(u(\mu+\rho), h)=(\lambda+\rho, h)+\sum_{\alpha \in A(\lambda)} k_{\alpha}(\alpha, h)=(\lambda+\rho, h) .
$$

Hence $(\mu, h) \geq(\lambda, h)$. Thus, in fact $(\lambda, h)=(\mu, h)$.

That implies that $(w(\lambda+\rho), h)=(\lambda+\rho, h)$ and $I \subset \Delta_{1}^{+}(\mathfrak{l})$. Since $(\lambda+\rho, \check{\beta})>0$ for all $\beta \in \Delta_{0}^{+}-\Delta(\mathfrak{l}), w$ belongs to the Weyl group of $\mathfrak{l}_{0}$. It follows from the construction of $w$ in the proof of Lemma 3 that $w=$ id and $i=0$. By Lemma $2, L_{\mu}$ is a subquotient of $\mathcal{U}(\mathfrak{g}) \otimes_{\mathcal{U}(\mathfrak{p})} L_{\lambda}(\mathfrak{p})$. Therefore $\mu=\lambda$.

Note that the proof of the above lemma implies the following

Corollary 2. - Let $\lambda$ be a dominant weight, $\mathfrak{p}$ be admissible for $\chi_{\lambda}$ and $h \in \mathfrak{h}^{*}$ be such that $\Delta(\mathfrak{p})=\{\alpha \in \Delta \mid(\alpha, h) \geq 0\}$. If $L_{\mu}$ occurs in $\Gamma_{i}\left(G / P, L_{\lambda}(\mathfrak{p})\right)$ with non-zero multiplicity, then $(\mu, h) \leq(\lambda, h)$. If, in addition, $(\lambda+\rho, \check{\beta})>0$ for all $\beta \in \Delta_{0}^{+}-\Delta(\mathfrak{l})$, then $(\mu, h)=(\lambda, h)$ implies $i=0$ and $\mu=\lambda$. 


\section{INDUCTION FOR GEOMETRIC INDUCTION}

We choose two parabolic subalgebras of $\mathfrak{g}$ containing $\mathfrak{b}$ such that $\mathfrak{b} \subset \mathfrak{p} \subset \mathfrak{q} \subset \mathfrak{g}$.

The aim of the present section is to relate, for a $\mathfrak{p}$-dominant weight $\mu$, the cohomology $\Gamma_{\bullet}\left(G / Q, L_{\lambda}(\mathfrak{q})\right)$ with both $\Gamma_{\bullet}\left(G / P, L_{\lambda}(\mathfrak{p})\right)$ and $\Gamma_{\bullet}\left(Q / P, L_{\mu}(\mathfrak{p})\right)$.

Definition 4. - For $A, B$ any $P, Q, G$ such that $B \subset A$, we define the Poincaré polynomial in the variable $z$ :

$$
K_{A, B}^{\lambda, \mu}(z):=\sum_{i \geq 0}\left[\Gamma_{i}\left(A / B, L_{\lambda}(\mathfrak{b})\right): L_{\mu}(\mathfrak{a})\right] z^{i} .
$$

We denote by ${ }^{i} K_{A, B}^{\lambda, \mu}$ the coefficient of $z^{i}$.

The following result was first stated in [25]:

Proposition 1. (Euler caracteristic formula) - Let $\mathfrak{p}$ be a parabolic subalgebra of $\mathfrak{g}$, denote by $\mathfrak{l}$ its Levi part and set

$$
D_{0}=\Pi_{\alpha \in \Delta_{0}^{+}}\left(e^{\alpha / 2}-e^{-\alpha / 2}\right), D_{1}=\Pi_{\alpha \in \Delta_{1}^{+}}\left(e^{\alpha / 2}+e^{-\alpha / 2}\right), D=\frac{D_{1}}{D_{0}} .
$$

For any $\mathfrak{p}$-dominant weight $\lambda$, one has :

$$
\sum_{\mu} K_{G, P}^{\lambda, \mu}(-1) C h\left(L_{\mu}\right)=D \sum_{w \in W} \varepsilon(w) w\left(\frac{e^{\rho} C h\left(L_{\lambda}(\mathfrak{p})\right)}{\Pi_{\alpha \in \Delta_{1}^{+}(\mathfrak{l})}\left(1+e^{-\alpha}\right)}\right) .
$$

Proof. - The left hand side of the formula is

$$
\sum_{i=0}^{\operatorname{dim} G_{0} / P_{0}}(-1)^{i} C h\left(H^{i}\left(G / P, \mathcal{L}_{\lambda}(\mathfrak{p})^{*}\right) .\right.
$$

Using Definition 1, one gets that the left hand side becomes

$$
\sum_{i=0}^{\operatorname{dim} G_{0} / P_{0}}(-1)^{i} C h\left(H^{i}\left(G_{0} / P_{0},\left(\oplus_{k} \tilde{\mathcal{L}}_{\lambda}(\mathfrak{p})^{k}\right)^{*}\right)^{*}\right) .
$$

We use the classical Borel-Weil-Bott theory to get

$$
\sum_{i=0}^{\operatorname{dim} G_{0} / P_{0}}(-1)^{i} C h\left(H^{i}\left(G_{0} / P_{0},\left(\oplus_{k} \tilde{\mathcal{L}}_{\lambda}(\mathfrak{p})^{k}\right)^{*}\right)^{*}\right)=\frac{1}{D_{0}} \sum_{w \in W} \varepsilon(w) w\left(C h\left(\oplus_{k} \tilde{\mathcal{L}}_{\lambda}(\mathfrak{p})^{k}\right) e^{\rho_{0}}\right) .
$$

Remember now that $\oplus_{k} \tilde{\mathcal{L}}_{\lambda}(\mathfrak{p})^{k}=\mathcal{I}_{G_{0} / P_{0}}\left(\mathcal{L}_{\lambda}(\mathfrak{p}) \otimes S^{\bullet}\left(\mathfrak{g} /\left(\mathfrak{g}_{0} \oplus \mathfrak{p}_{1}\right)\right)\right)$. A direct computation gives the proposition.

Theorem 1. - One has :

$$
K_{G, P}^{\lambda, \mu}(-1)=\sum_{\nu} K_{Q, P}^{\lambda, \nu}(-1) K_{G, Q}^{\nu, \mu}(-1) .
$$

Proof. - Denote by $\pi: G / P \longrightarrow G / Q$ the canonical projection. The fibre of $\pi$ is isomorphic to $Q / P$. Consider the derived functor (of sheaves) $R^{\bullet} \pi_{*}$, it transforms $\mathcal{L}_{\lambda}(\mathfrak{p})^{*}$ into a complex of sheaves $R^{\bullet} \pi_{*}\left(\mathcal{L}_{\lambda}(\mathfrak{p})^{*}\right)$ over $G / Q$.

Take an injective resolution $\mathcal{L}_{k}$ of $\mathcal{L}_{\lambda}(\mathfrak{p})^{*}$, over $G / P$ and then an injective resolution of $\pi_{*}\left(\mathcal{L}_{k}\right)$ over $G / Q$. This gives a bicomplex of sheaves over $G / Q$, and its cohomology is isomorphic to $H^{\bullet}\left(G / P, \mathcal{L}_{\lambda}(\mathfrak{p})^{*}\right)$. 
On the other hand, the Leray spectral sequence of this bicomplex has the term

$$
E_{2}^{p, q}=H^{p}\left(G / Q, R^{q} \pi_{*}\left(\mathcal{L}_{\lambda}(\mathfrak{p})^{*}\right)\right) .
$$

One has $([15])$

$$
R^{q} \pi_{*} \mathcal{L}_{\lambda}(\mathfrak{p})^{*}=H^{q}\left(Q / P, \mathcal{L}_{\lambda}(\mathfrak{p})_{\mid \text {fibre }}^{*}\right) .
$$

By definition of the coefficient ${ }^{q} K_{A, B}^{\lambda, \mu}$, we have the following identities in the Grothendieck groups:

$$
\begin{gathered}
{\left[H^{q}\left(Q / P, \mathcal{L}_{\lambda}(\mathfrak{p})_{\mid f i b r e}^{*}\right)\right]=\sum_{\nu}{ }^{q} K_{Q, P}^{\lambda, \nu}\left[L_{\nu}(\mathfrak{q})^{*}\right],} \\
{\left[E_{2}^{p, q}\right]=\sum_{\mu} \sum_{\nu}{ }^{p} K_{G, Q}^{\nu, \mu} K_{Q, P}^{\lambda, \nu}\left[L_{\mu}^{*}\right] .}
\end{gathered}
$$

The theorem follows when one computes the Euler characteristic.

\section{BLOCKS}

Recall that we let $\mathcal{U}(\mathfrak{g})$ be the universal enveloping algebra of $\mathfrak{g}$ and $\mathcal{Z}(\mathfrak{g})$ be its center. For every weight $\lambda$, we write $\chi_{\lambda}$ for the corresponding central character.

The aim of the present section is to prove the following:

Theorem 2. - Let $\lambda$ be a dominant weight with atypicality degree $k$, then the block $\mathcal{F} \chi_{\lambda}$ is equivalent to the maximal atypical block of $\mathfrak{g}_{k}$ containing the trivial module, where $\mathfrak{g}_{k}$ is the following:

$$
\begin{aligned}
& \text { if } \mathfrak{g}=\mathfrak{g l}(m, n) \text { then } \mathfrak{g}_{k}=\mathfrak{g l}(k, k) \\
& \text { if } \mathfrak{g}=\mathfrak{o s p}(2 m+1,2 n) \text { then } \mathfrak{g}_{k}=\mathfrak{o s p}(2 k+1,2 k) \\
& \text { if } \mathfrak{g}=\mathfrak{o s p}(2 m, 2 n) \text { then } \mathfrak{g}_{k}=\mathfrak{o} \mathfrak{s p}(2 k, 2 k) \text { or } \mathfrak{o s p}(2 k+2,2 k)
\end{aligned}
$$

In what follows the Borel subalgebra $\mathfrak{b}$ is such that every simple root of $\mathfrak{b}_{0}$ is either a simple root of $\mathfrak{b}$ or a sum of two odd simple roots of $\mathfrak{b}$.

Lemma 6. - A weight $\lambda$ is dominant, i.e. $L_{\lambda}$ is finite-dimensional if and only if, for any simple root $\alpha$ of $\mathfrak{b}_{0}$,

- $(\lambda+\rho, \check{\alpha}) \in \mathbb{Z}_{>0}$ if $\alpha$ or $\frac{\alpha}{2}$ is simple in $\mathfrak{b}$;

- if $\alpha=\gamma+\beta$ is a sum of two isotropic simple roots then $(\lambda+\rho, \check{\alpha}) \in \mathbb{Z}_{>0}$ or $(\lambda+\rho, \beta)=$ $(\lambda+\rho, \gamma)=0$

- Finally if $\alpha=\beta+\gamma$ where $\gamma$ is an odd isotropic simple root and $\beta$ is an odd nonisotropic simple root, then $(\lambda+\rho, \check{\alpha}) \in \mathbb{Z}_{>0}$ or $(\lambda+\rho, \check{\alpha})=-1$ and $(\lambda+\rho, \gamma)=0$.

Proof. - For an arbitrary Borel subalgebra $\mathfrak{b}^{\prime}$ containing $\mathfrak{b}_{0}$ let $\lambda\left(\mathfrak{b}^{\prime}\right)$ denote the highest weight of $L_{\lambda}$ with respect to $\mathfrak{b}^{\prime}$ and $\rho\left(\mathfrak{b}^{\prime}\right)$ be the analogue of $\rho$ for $\mathfrak{b}^{\prime}$. A weight $\lambda$ is dominant (see [27]) if for any simple root $\alpha$ of $\mathfrak{b}_{0}$ there exists a Borel subalgebra $\mathfrak{b}^{\prime}$ such that $\alpha$ or $\frac{\alpha}{2}$ is simple in $\mathfrak{b}^{\prime}$ and $\left(\lambda\left(\mathfrak{b}^{\prime}\right)+\rho\left(\mathfrak{b}^{\prime}\right), \check{\alpha}\right) \in \mathbb{Z}_{>0}$.

If $\alpha$ or $\frac{\alpha}{2}$ is a simple root of $\mathfrak{b}$, then we are in the situation of the first two cases and the statement is straightforward. If $\alpha=\beta+\gamma$, then choose $\mathfrak{b}^{\prime}$ obtained by odd reflection with respect to $\gamma$. Then the statement follows from the following formulae

$$
\begin{gathered}
\lambda\left(\mathfrak{b}^{\prime}\right)+\rho\left(\mathfrak{b}^{\prime}\right)=\lambda+\rho \text { if }(\lambda+\rho, \gamma) \neq 0, \\
\lambda\left(\mathfrak{b}^{\prime}\right)+\rho\left(\mathfrak{b}^{\prime}\right)=\lambda+\rho+\gamma \text { if }(\lambda+\rho, \gamma)=0 .
\end{gathered}
$$


Remark - Note that our choice of $\mathfrak{b}$ implies that for any simple root $\alpha$ of $\mathfrak{b}_{0},(\rho, \check{\alpha})=$ $1,0,-1$ if $\alpha$ is a simple root of $\mathfrak{b}$, a sum of two isotropic simple roots or a sum of one isotropic and one non-isotropic odd simple roots respectively. The latter case is possible only for $\mathfrak{o s p}(2 m+1,2 n)$. In particular, in the cases of $\mathfrak{g l}(m, n)$ and $\mathfrak{o s p}(2 m, 2 n)$, every parabolic subalgebra of $\mathfrak{g}$ containing $\mathfrak{b}$ is admissible for all central characters.

The above conditions on a Borel subalgebra determine $\mathfrak{b}$ uniquely up to an automorphism of $\mathfrak{g}$ if $\mathfrak{g}=\mathfrak{o s p}(m, 2 n)$. In the case of $\mathfrak{g}=\mathfrak{g l}(m, n)$ we choose the distinguished $\mathfrak{b}$.

Here we list the simple roots for our choice of Borel subalgebra:

- If $\mathfrak{g}=\mathfrak{g l}(m, n), m \geq n$, the simple roots are

$$
\begin{gathered}
\varepsilon_{1}-\varepsilon_{2}, \varepsilon_{2}-\varepsilon_{3}, \ldots, \varepsilon_{m}-\delta_{1}, \delta_{1}-\delta_{2}, \ldots, \delta_{n-1}-\delta_{n}, \\
\rho=\frac{m-n-2}{2} \varepsilon_{1}+\frac{m-n-4}{2} \varepsilon_{2}+\ldots+\frac{m-n}{2} \varepsilon_{m}+\frac{m+n}{2} \delta_{1}+\ldots+\frac{m-n+2}{2} \delta_{n} ;
\end{gathered}
$$

- If $\mathfrak{g}=\mathfrak{o s p}(2 m+1,2 n)$ and $m \geq n$, the simple roots are

$$
\begin{gathered}
\varepsilon_{1}-\varepsilon_{2}, \ldots, \varepsilon_{m-n+1}-\delta_{1}, \delta_{1}-\varepsilon_{m-n+2}, \ldots, \varepsilon_{m}-\delta_{n}, \delta_{n}, \\
\rho=\left(m-n-\frac{1}{2}\right) \varepsilon_{1}+\left(m-n-\frac{3}{2}\right) \varepsilon_{2}+\ldots+\frac{1}{2} \varepsilon_{m-n}-\frac{1}{2} \varepsilon_{m-n-1}-\ldots-\frac{1}{2} \varepsilon_{m}+\frac{1}{2} \delta_{1}+\ldots+\frac{1}{2} \delta_{n} ;
\end{gathered}
$$

- If $\mathfrak{g}=\mathfrak{o s p}(2 m+1,2 n)$ and $m<n$, the simple roots are

$$
\begin{gathered}
\delta_{1}-\delta_{2}, \ldots, \delta_{n-m}-\delta_{1}, \delta_{1}-\varepsilon_{n-m+1}, \ldots, \varepsilon_{m}-\delta_{n}, \delta_{n}, \\
\rho=-\frac{1}{2} \varepsilon_{1}-\ldots-\frac{1}{2} \varepsilon_{m}+\left(n-m+\frac{1}{2}\right) \delta_{1}+\left(n-m-\frac{1}{2}\right) \delta_{2}+\ldots+\frac{1}{2} \delta_{n-m+1}+\ldots+\frac{1}{2} \delta_{n} ;
\end{gathered}
$$

- If $\mathfrak{g}=\mathfrak{o s p}(2 m, 2 n)$ and $m>n$, the simple roots are

$$
\begin{gathered}
\varepsilon_{1}-\varepsilon_{2}, \ldots, \varepsilon_{m-n}-\delta_{1}, \delta_{1}-\varepsilon_{m-n+1}, \ldots, \delta_{n}-\varepsilon_{m}, \delta_{n}+\varepsilon_{m}, \\
\rho=(m-n) \varepsilon_{1}+(m-n-1) \varepsilon_{2}+\ldots+\varepsilon_{m-n+1}
\end{gathered}
$$

- If $\mathfrak{g}=\mathfrak{o s p}(2 m, 2 n)$ and $m \leq n$, the simple roots are

$$
\begin{gathered}
\delta_{1}-\delta_{2}, \ldots, \delta_{n-m+1}-\varepsilon_{1}, \varepsilon_{1}-\delta_{n-m+2}, \ldots, \delta_{n}-\varepsilon_{m}, \delta_{n}+\varepsilon_{m}, \\
\rho=(n-m) \delta_{1}+(n-m-1) \delta_{2}+\ldots+\delta_{n-m+1} .
\end{gathered}
$$

Corollary 3. Let

$$
\lambda+\rho=a_{1} \varepsilon_{1}+\ldots+a_{m} \varepsilon_{m}+b_{1} \delta_{1}+\ldots+b_{n} \delta_{n} .
$$

Then $\lambda$ is integral iff $a_{i}, b_{j} \in \mathbb{Z}$ for $\mathfrak{g}=\mathfrak{g l}(m, n)$ or $\mathfrak{o s p}(2 m, 2 n), a_{i}, b_{j} \in \frac{1}{2}+\mathbb{Z}$ for $\mathfrak{g}=\mathfrak{o s p}(2 m+1,2 n)$. Furthermore, $\lambda$ is dominant iff the following conditions hold

- If $\mathfrak{g}=\mathfrak{g l}(m, n)$,

$$
a_{1}>a_{2}>\ldots>a_{m} \text { and } b_{1}>b_{2}>\ldots>b_{n} ;
$$

- If $\mathfrak{g}=\mathfrak{o s p}(2 m+1,2 n)$, either

$$
a_{1}>a_{2}>\ldots>a_{m} \geq \frac{1}{2}, b_{1}>b_{2}>\ldots>b_{n} \geq \frac{1}{2},
$$

or

$$
a_{1}>a_{2}>\ldots>a_{m-l-1}>a_{m-l}=\ldots=a_{m}=-\frac{1}{2}
$$

and $b_{1}>b_{2}>\ldots>b_{n-l-1} \geq b_{n-l}=\ldots=b_{n}=\frac{1}{2}$ for some $0 \leq l \leq \min (m, n)$; 
- If $\mathfrak{g}=\mathfrak{o s p}(2 m, 2 n)$, either

$$
a_{1}>a_{2}>\ldots>a_{m-1}>\left|a_{m}\right| \text { and } b_{1}>b_{2}>\ldots>b_{n}>0,
$$

or

$$
a_{1}>a_{2}>\ldots>a_{m-l-1} \geq a_{m-l}=\ldots=a_{m}=0
$$

and $b_{1}>b_{2}>\ldots>b_{n-l-1}>b_{n-l}=\ldots=b_{n}=0$ for some $0 \leq l \leq \min (m, n)$.

Let $\chi=\chi_{\nu}$ be a central character with atypicality degree $k$. Choose a self-commuting element $x=\sum_{\alpha \in A(\nu)} X_{\alpha}$. Let $C(x)$ denote the centralizer of $x$ in $\mathfrak{g}$. Then $[x, \mathfrak{g}]$ is an ideal in $C(x)$ and one can choose a reductive subalgebra $\mathfrak{g}_{x} \subset C(x)$ such that $C(x)=\mathfrak{g}_{x} \oplus[x, \mathfrak{g}]$ (see [9]). This choice is canonical if the Cartan subalgebra is fixed. Denote by $\mathcal{U}(\mathfrak{g})^{x}$ the set of $a d_{x}$-invariants in $\mathcal{U}(\mathfrak{g})$. One can prove $\left([9)\right.$ that $\mathcal{U}(\mathfrak{g})^{x}=\mathcal{U}\left(\mathfrak{g}_{x}\right) \oplus \mathcal{U}\left(\mathfrak{g}_{x}\right)[x, \mathcal{U}(\mathfrak{g})]$. Consider the projection $p: \mathcal{U}(\mathfrak{g})^{x} \rightarrow \mathcal{U}\left(\mathfrak{g}_{x}\right)$ with the kernel $\mathcal{U}\left(\mathfrak{g}_{x}\right)[x, \mathcal{U}(\mathfrak{g})]$. The restriction of $p$ to the center of $\mathcal{Z}(\mathfrak{g})$ defines a homomorphism $\mathcal{Z}(\mathfrak{g}) \rightarrow \mathcal{Z}\left(\mathfrak{g}_{x}\right)$. Consider the dual map

$$
p^{*}: \operatorname{Hom}\left(\mathcal{Z}\left(\mathfrak{g}_{x}\right), \mathbb{C}\right) \rightarrow \operatorname{Hom}(\mathcal{Z}(\mathfrak{g}), \mathbb{C}) .
$$

It was shown in [9] that the preimage $\left(p^{*}\right)^{-1}(\chi)$ consists of one central character $\chi^{\prime} \in$ $\operatorname{Hom}\left(\mathcal{Z}\left(\mathfrak{g}_{x}\right), \mathbb{C}\right)$ unless $\mathfrak{g}=\mathfrak{o} \mathfrak{s}(2 m, 2 n)$. If $\mathfrak{g}=\mathfrak{o s p}(2 m, 2 n)$ then $\left(p^{*}\right)^{-1}(\chi)$ might consist of two central characters $\chi^{\prime}$ and $\chi^{\prime \prime}$ such that one is obtained from another by an involutive outer automorphism of $\mathfrak{g}_{x} \simeq \mathfrak{o s p}(2 m-2 k, 2 n-2 k)$ (induced by an automorphism of $\mathfrak{o}(2 m-2 k))$. In the latter case by $\chi^{\prime}$ we denote the central character corresponding to a dominant weight with non-negative marks.

Terminology - We call $\chi^{\prime}$ the core of $\chi$.

If $\mathfrak{h}_{x}=\mathfrak{h} \cap \mathfrak{g}_{x}, \rho^{\prime}=\frac{1}{2}\left(\sum_{\alpha \in \Delta_{0}^{+}\left(\mathfrak{g}_{x}\right)} \alpha-\sum_{\alpha \in \Delta_{1}^{+}\left(\mathfrak{g}_{x}\right)} \alpha\right)$, then $\chi^{\prime}=\chi_{\mu}$, where $\mu+\rho^{\prime}$ is the restriction of $\nu+\rho$ to $\mathfrak{h}_{x}$. It is a simple but very important observation that the central character $\chi$ is uniquely determined by its core $\chi^{\prime}$.

Lemma 6 and Corollary 3 imply the following

Lemma 7. - The core $\chi^{\prime}$ is a typical dominant central character of $\mathfrak{g}_{x}$.

Assume that $\chi$ has degree of atypicality $k>0$. Then, independently of the choice of $\lambda$ such that $\chi_{\lambda}=\chi$, the Lie superalgebra $\mathfrak{g}_{x}$ is isomorphic to one of the following (see [9])

- if $\mathfrak{g}=\mathfrak{g l}(m, n)$ then $\mathfrak{g}_{x} \simeq \mathfrak{g l}(p, q)$ with $p=m-k, q=n-k$;

- if $\mathfrak{g}=\mathfrak{o s p}(2 m+1,2 n)$ then $\mathfrak{g}_{x} \simeq \mathfrak{o} \mathfrak{s p}(2 p+1,2 q)$ with $p=m-k, q=n-k$;

- if $\mathfrak{g}=\mathfrak{o s p}(2 m, 2 n)$ then $\mathfrak{g}_{x}=\mathfrak{o s p}(2 p, 2 q)$ with $p=m-k, q=n-k$.

In all cases it will be convenient to encode the core $\chi^{\prime}$ by the corresponding dominant typical weight $\mu+\rho^{\prime}$ of $\mathfrak{g}_{x}$. In what follows we write

$$
\chi^{\prime}:=\mu+\rho^{\prime}=a_{1} \varepsilon_{1}+\cdots+a_{p} \varepsilon_{p}+b_{1} \delta_{1}+\cdots+b_{q} \delta_{q},
$$

where $a_{i}, b_{j}$ satisfy the additional assumptions of dominance and typicality with respect to $\mathfrak{g}_{x}$ (and additional positivity condition for $\mathfrak{g}_{x} \simeq \mathfrak{o} \mathfrak{s p}(2 p, 2 q)$ ), more precisely

$$
\begin{gathered}
a_{1}>\cdots>a_{p}, b_{1}>\cdots>b_{q}, \\
a_{i}, b_{j} \in \mathbb{Z}, a_{i} \neq-b_{j} \text { if } \mathfrak{g}=\mathfrak{g l}(m, n), \\
a_{i}, b_{j} \in \frac{1}{2}+\mathbb{Z}_{\geq 0}, a_{i} \neq b_{j} \text { if } \mathfrak{g}=\mathfrak{o s p}(2 m+1,2 n), \\
a_{i} \in \mathbb{Z}_{\geq 0}, b_{j} \in \mathbb{Z}_{>0}, a_{i} \neq b_{j} \text { if } \mathfrak{g}=\mathfrak{o s p}(2 m, 2 n) .
\end{gathered}
$$


We call the numbers $a_{i}, b_{j}$ the marks of the core.

Now we define $\mathfrak{g}_{\chi} \subset \mathfrak{g}$ corresponding to a connected sub-Dynkin diagram containing the last node(s) of the diagram of $\mathfrak{g}$ in the following way:

- if $\mathfrak{g}=\mathfrak{g l}(m, n)$ then $\mathfrak{g}_{\chi} \simeq \mathfrak{g l}(k, k)$;

- if $\mathfrak{g}=\mathfrak{o s p}(2 m+1,2 n)$ then $\mathfrak{g}_{\chi} \simeq \mathfrak{o s p}(2 k+1,2 k)$;

- if $\mathfrak{g}=\mathfrak{o s p}(2 m, 2 n)$ and $a_{p}>0$, then $\mathfrak{g}_{\chi} \simeq \mathfrak{o s p}(2 k, 2 k)$;

- if $\mathfrak{g}=\mathfrak{o s p}(2 m, 2 n)$ and $a_{p}=0$, then $\mathfrak{g}_{\chi} \simeq \mathfrak{o s p}(2 k+2,2 k)$.

Let $\mathfrak{p}$ be the parabolic subalgebra containing $\mathfrak{b}$ whose Levi part is $\mathfrak{l}=\mathfrak{g}_{\chi}+\mathfrak{h}$. Assume that $\chi$ is such that $\mathfrak{p}$ is admissible for $\chi$ and $\lambda$ is a dominant weight such that $\chi_{\lambda}=\chi$.

Terminology - If in addition $\lambda$ is $\mathfrak{p}$-typical, we call $\lambda$ stable.

Let $\mathcal{F}_{\leq \lambda}^{\chi}$ denote the subcategory in $\mathcal{F}^{\chi}$ consisting of all $\mathfrak{g}$-modules with all weights $\leq \lambda$. It is not hard to see that if $\lambda$ is stable then the highest weight of any simple $\mathfrak{g}$-module in $\mathcal{F}_{\leq \lambda}^{\chi}$ is also stable. By $\mathcal{F}_{\leq \lambda}^{\chi}(\mathfrak{l})$ we denote the corresponding truncated category of $\mathfrak{l}$-modules with central character from the set $\Phi^{-1}(\chi)$ ( $\Phi$ was defined just before Lemma 44).

Define the functors Res : $\mathcal{F}^{\chi} \rightarrow \mathcal{F}^{\chi}(\mathfrak{l})$ and Ind $: \mathcal{F}^{\chi}(\mathfrak{l}) \rightarrow \mathcal{F}^{\chi}$ by

$$
\operatorname{Res} N=N^{\mathfrak{m}}, \text { Ind } M=\Gamma_{0}(G / P, M),
$$

recall that $\mathfrak{m}$ stands for the nilpotent radical of $\mathfrak{p}$.

Lemma 8. - Assume that $\lambda$ is stable. Then the functors Res and Ind establish an equivalence between the categories $\mathcal{F}_{\leq \lambda}^{\chi}$ and $\mathcal{F}_{\leq \lambda}^{\chi}(\mathfrak{p})$.

Proof. - The statement easily follows from the typical lemma, (Lemma 5). Indeed for any simple module $L_{\mu}(\mathfrak{p}) \in \mathcal{F}_{\leq \lambda}^{\chi}(\mathfrak{p})$, Ind $L_{\mu}(\mathfrak{p})=\Gamma_{0}\left(G / P, L_{\mu}(\mathfrak{p})\right)=L_{\mu}$ is the unique simple quotient of $\mathcal{U}(\mathfrak{g}) \otimes_{\mathcal{U}(\mathfrak{p})} L_{\mu}(\mathfrak{p})$. All the higher cohomology groups vanish. Therefore Ind is an exact functor which maps a simple module to a simple module. Clearly,

$$
\operatorname{IndRes}\left(L_{\mu}(\mathfrak{p})\right) \simeq L_{\mu}(\mathfrak{p}), \operatorname{ResInd}\left(L_{\mu}\right) \simeq L_{\mu},
$$

therefore the lemma holds.

Our next step in the proof of Theorem 2 is "to move" any simple module to $\mathcal{F}_{\leq \lambda}^{\chi}$ using translation functors. Recall the definition of translation functors (see [1]): let $V$ be a finite-dimensional $\mathfrak{g}$-module. One defines a functor $T(V)^{\chi, \tau}: \mathcal{F}^{\chi} \rightarrow \mathcal{F}^{\tau}$ by $T(V)^{\chi, \tau}(M)=$ $(M \otimes V)^{\tau}$. It is not difficult to see that $T(V)^{\chi, \tau}$ is exact and $T\left(V^{*}\right)^{\tau, \chi}$ is left adjoint to $T(V)^{\chi, \tau}$. The following lemma is also straightforward (see for example [1]).

Lemma 9. - If both $T\left(V^{*}\right)^{\tau, \chi}$ and $T(V)^{\chi, \tau}$ move a simple module to a simple module, then they establish an equivalence between the categories $\mathcal{F}^{\chi}$ and $\mathcal{F}^{\tau}$.

Lemma 10. - Let $\tau$ and $\chi$ be central characters. Assume

- for any dominant $\mu$ with $\chi_{\mu}=\chi$ there exists a unique weight $\gamma$ of $V$, such that $\mu+\gamma$ is dominant and $\chi_{\mu+\gamma}=\tau$;

- for any dominant $\nu$ with $\chi_{\nu}=\tau$ there exists a unique weight $\gamma^{\prime}$ of $V^{*}$ such that $\nu+\gamma^{\prime}$ is dominant and $\chi_{\nu+\gamma^{\prime}}=\chi$;

- the multiplicities of $\gamma$ in $V$ and $\gamma^{\prime}$ in $V^{*}$ are 1.

Then $T(V)^{\chi, \tau}$ and $T\left(V^{*}\right)^{\tau, \chi}$ establish an equivalence between the categories $\mathcal{F}^{\chi}$ and $\mathcal{F}^{\tau}$.

Proof. - It suffices to prove that $T(V)^{\chi, \tau}\left(L_{\mu}\right)=L_{\mu+\gamma}$ for any $L_{\mu} \in \mathcal{F}^{\chi}$.

First we note that a $\mathfrak{b}$-singular vector in $\left(L_{\mu} \otimes V\right)^{\tau}$ has weight $\mu+\gamma$. Hence $T(V)^{\chi, \tau}\left(L_{\mu}\right)$ has a unique up to proportionality $\mathfrak{b}$-singular vector. Since $T(V)^{\chi, \tau}\left(L_{\mu}\right)$ is contragredient, it is either zero or $L_{\mu+\gamma}$. It is left to prove that $T(V)^{\chi, \tau}\left(L_{\mu}\right) \neq 0$. 
Let $C_{\mu}$ denote the one-dimensional $\mathfrak{b}$-module of weight $\mu$. Since

$$
\Gamma_{0}\left(G / B, C_{\mu} \otimes V\right)=\Gamma_{0}\left(G / B, C_{\mu}\right) \otimes V,
$$

and $\left(C_{\mu} \otimes V\right)^{\Phi^{-1}(\tau)}$ has only one dominant component $C_{\mu+\gamma}$, Corollary 1 and Lemma 2 imply the isomorphism

$$
T(V)^{\chi, \tau}\left(\Gamma_{0}\left(G / B, C_{\mu}\right)\right) \simeq \Gamma_{0}\left(G / B, C_{\mu+\gamma}\right) .
$$

Assume that $T(V)^{\chi, \tau}\left(L_{\mu}\right)=0$. Then, since $T(V)^{\chi, \tau}$ is exact, there must be a simple subquotient $L_{\mu^{\prime}}$ in $\left.\Gamma_{0}\left(G / B, C_{\mu}\right)\right)$ such that $T(V)^{\chi, \tau}\left(L_{\mu^{\prime}}\right)=L_{\mu+\gamma}$. Therefore $\mu^{\prime}+\delta=$ $\mu+\gamma$ for some weight $\delta$ of $V$. But the conditions of the lemma imply that $\mu^{\prime}=\mu$. Contradiction.

Let $E$ denote the standard $\mathfrak{g}$-module.

Lemma 11. - Let $\chi$ be a central character with degree of atypicality $k>0$ and core $\chi^{\prime}=a_{1} \varepsilon_{1}+\ldots+a_{p} \varepsilon_{p}+b_{1} \delta_{1} \ldots+b_{q} \delta_{q}$. Let $V=E$ or $E^{*}$ and $\delta$ be a weight of $V$. Assume that $\chi^{\prime}+\delta$ satisfies the conditions (3)-(6) and in addition for $\mathfrak{g}=\mathfrak{o s p}(2 m, 2 n)$ the numbers of zero marks in $\chi^{\prime}+\delta$ and in $\chi^{\prime}$ are the same. Let $\tau$ be the central character such that $\tau^{\prime}=\chi^{\prime}+\delta$. Then $T(V)^{\chi, \tau}$ and $T\left(V^{*}\right)^{\tau, \chi}$ establish an equivalence between $\mathcal{F} \chi$ and $\mathcal{F}^{\tau}$.

Proof. - Since $V=E$ or $E^{*}$, every weight $\gamma$ of $V$ has multiplicity 1, moreover $\gamma= \pm \varepsilon_{i}, \pm \delta_{j}$ or 0 . The proof can be reduced to checking the conditions of Lemma 10 for any $L_{\mu} \in \mathcal{F}^{\chi}$. We will consider here the most tedious case of $\mathfrak{g}=\mathfrak{o} \mathfrak{s p}(2 m, 2 n), \delta= \pm \varepsilon_{i}$, the other cases are completely analogous and we leave them to the reader.

Since $\chi_{\mu}=\chi$ there are the following two possibilities: either $\left(\mu+\rho, \varepsilon_{j}\right)=a_{i}$ for a unique $j \leq m$ or $\left(\mu+\rho, \varepsilon_{m}\right)=-a_{i}$ (in the latter case $\left(\mu+\rho, \varepsilon_{j}\right)>a_{i}$ for all $j<m$ ).

Let $\delta=\varepsilon_{i}$. In the former case take $\nu=\mu+\varepsilon_{j}$ if $\left(\mu+\rho, \varepsilon_{j-1}\right)>a_{i}+1$. If $\left(\mu+\rho, \varepsilon_{j-1}\right)=$ $a_{i}+1$, there exists $l$ such that $\left(\mu+\rho, \varepsilon_{j-1}+\delta_{l}\right)=0$ and one should take $\nu=\mu-\delta_{l}$. In the latter case take $\nu=\mu-\varepsilon_{m}$ if $\left(\mu+\rho, \varepsilon_{m-1}\right)>a_{i}+1$. If $\left(\mu+\rho, \varepsilon_{m-1}\right)=a_{i}+1$, there exists $k$ such that $\left(\mu+\rho, \varepsilon_{m-1}+\delta_{l}\right)=0$, take $\nu=\mu-\delta_{l}$.

Now deal similarly with the case $\delta=-\varepsilon_{i}$. In the former case take $\nu=\mu-\varepsilon_{j}$ if $\left|\left(\mu+\rho, \varepsilon_{j+1}\right)\right|<a_{i}-1$. If $\left(\mu+\rho, \varepsilon_{j+1}\right)=a_{i}-1$, there exists $l$ such that $\left(\mu+\rho, \varepsilon_{j+1}+\delta_{l}\right)=0$ and choose $\nu=\mu+\delta_{l}$. If $\left(\mu+\rho, \varepsilon_{j+1}\right)=-a_{i}+1$, then $j+1=m$, there exists $k$ such that $\left(\mu+\rho, \varepsilon_{m}-\delta_{l}\right)=0$ and choose $\nu=\mu+\delta_{l}$. Finally in the latter case take $\nu=\mu+\varepsilon_{m}$.

Lemma 12. - Let $k$ be the degree of atypicality of $\chi, \chi^{\prime}=a_{1} \varepsilon_{1}+\ldots+a_{p} \varepsilon_{p}+b_{1} \delta_{1} \ldots+b_{q} \delta_{q}$. Let $\mathfrak{p}$ be the parabolic subalgebra with Levi part $\mathfrak{l}=\mathfrak{h}+\mathfrak{g}_{\chi}$ which contains $\mathfrak{b}$. Let $\lambda$ be a dominant weight such that $\chi_{\lambda}=\chi$. There exist a central character $\tau$ such that $\mathfrak{p}$ is admissible for $\tau$, and a dominant stable weight $\mu$, such that $\chi_{\mu}=\tau$ and $\mathcal{F}_{\leq \lambda}^{\chi}$ is equivalent to $\mathcal{F}_{\leq \mu}^{\tau}$.

Proof. - Let

$$
\chi^{\prime}=a_{1} \varepsilon_{1}+\ldots+a_{p} \varepsilon_{p}+b_{1} \delta_{1}+\ldots+b_{q} \delta_{q} .
$$

If $a_{1}>b_{1}$ in osp case, $a_{1}>-b_{q}$ in $\mathfrak{g l}$ case, put $\chi_{1}^{\prime}=\chi^{\prime}+\varepsilon_{1}$, let $\chi_{1}$ be the central character with core $\chi_{1}^{\prime}$. In this way proceed to increase $a_{1}$ so that it is bigger than the absolute value of any coordinate of $\lambda$ plus $p+q$. If $a_{1}<b_{1}$ in the osp case, increase $b_{1}$ in the same manner. In the $\mathfrak{g l}$ case, if $a_{1}<-b_{q}$ decrease $b_{q}$. After this, pick up the next mark in $\chi^{\prime}$ and increase (decrease) it following the same method to the absolute value of the previous mark -1. Proceed in the same manner with all marks of $\chi^{\prime}$ increasing the absolute value of each mark (except $a_{p}=0$ in the $\mathfrak{o s p}(2 m, 2 n)$ case). Call the resulting core $\tau^{\prime}$, and let $\tau$ be the corresponding central character. As follows from Lemma 11 the categories $\mathcal{F}^{\chi}$ 
and $\mathcal{F}^{\tau}$ are equivalent via a composition of translation functors, which we denote by $T$. Then $T\left(L_{\lambda}\right)=L_{\mu}$ and one can easily check that $\mu$ is stable and $\mathfrak{p}$-typical. Hence $\mathcal{F}_{\leq \lambda}^{\chi}$ is equivalent to $\mathcal{F}_{\leq \mu}^{\tau}$.

We would like to illustrate the above argument with few examples.

Let $\mathfrak{g}=\mathfrak{g l}(3,2), \lambda+\rho=(2,0 \mid 3,0,-1)$, then $\mu+\rho=(7,-1 \mid 1,-5,-6)$.

Let $\mathfrak{g}=\mathfrak{o s p}(5,4), \lambda+\rho=\left(\frac{5}{2},-\frac{1}{2} \mid \frac{5}{2}, \frac{3}{2}, \frac{1}{2}\right)$, then $\mu+\rho=\left(\frac{3}{2},-\frac{1}{2} \mid \frac{9}{2}, \frac{3}{2}, \frac{1}{2}\right)$.

Let $\mathfrak{g}=\mathfrak{o s p}(4,6), \lambda+\rho=(4,-2 \mid 3,2,1)$, then $\mu+\rho=(7,-1 \mid 6,5,1)$.

Lemma 12 and Lemma 8 imply Theorem 2. Indeed, by Lemma 12 for any dominant $\lambda$ with $\chi_{\lambda}=\chi$ the truncated category $\mathcal{F}_{\leq \lambda}^{\chi}$ is equivalent to the "stable" truncated category $\mathcal{F}_{\leq \mu}^{\tau}$ for a suitable choice of $\mu$. The latter category is equivalent to $\mathcal{F}_{\leq \mu}^{\tau}(\mathfrak{l})$ by Lemma 8 , Finally, $\mathcal{F}_{\leq \mu}^{\tau}(\mathfrak{l})$ is equivalent to the truncated part of the most atypical block of $\mathfrak{g}_{\chi}$ since $\mathfrak{l}$ is the direct sum of $\mathfrak{g}_{\chi}$ and a center. Since $\lambda$ is arbitrary one can extend this equivalence to the whole $\mathcal{F} \chi$.

\section{WEIGHT DIAGRAMS AND TRANSLATION FUNCTORS}

In this section we define an alternative way to describe dominant weights following Brundan and Stroppel. Their method allows one to visualize the action of the translation functors defined in the previous section.

Let $\mathbb{T} \subset \mathbb{R}$ be a discrete set, $X=\left(x_{1}, \ldots, x_{m}\right) \in \mathbb{T}^{m}, Y=\left(y_{1}, \ldots, y_{n}\right) \in \mathbb{T}^{n}$. A diagram $f_{X, Y}$ is a function defined on $\mathbb{T}$ whose values are multisets with elements $<,>, \times$ according to the following algorithm.

- Put the symbol $>$ in position $t$ for all $i$ such that $x_{i}=t$.

- Put the symbol $<$ in position $t$ for all $i$ such that $y_{i}=t$.

- If there are both $>$ and $<$ in the same position replace them by the symbol $\times$, repeat if possible.

Thus, $f_{X, Y}(t)$ may contain at most one of the two symbols $>,<$. We represent $f_{X, Y}$ by the picture with 0 standing in position $t$ whenever $f(t)$ is an empty set.

Let $\mathfrak{g}=\mathfrak{g l}(m, n)$. Let $\lambda$ be a dominant integral weight such that

$$
\lambda+\rho=a_{1} \varepsilon_{1}+\ldots+a_{m} \varepsilon_{m}+b_{1} \delta_{1}+\ldots+b_{n} \delta_{n} .
$$

Set $\mathbb{T}=\mathbb{Z}$

$$
X_{\lambda}=\left(a_{1}, \ldots, a_{m}\right), Y_{\lambda}=\left(-b_{1}, \ldots,-b_{n}\right) .
$$

The diagram $f_{\lambda}=f_{X_{\lambda}, Y_{\lambda}}$ is called the weight diagram of $\lambda$.

A diagram is the weight diagram of some dominant weight if and only if $f(t)$ is empty or is a just one element set since both sequences $a_{1}, \ldots, a_{m}$ and $b_{1}, \ldots, b_{m}$ are strictly decreasing and hence do not have repetitions.

Each dominant weight is uniquely determined by its weight diagram. The number of $<$ is $n$, the number of $>$ is $m$ (counting $\times$ as both $<$ and $>$ ). The number of $\times$ equals the degree of atypicality. Replacing all $\times$ in the diagram by zeros gives a diagram of the core. For example, the diagram

$$
\ldots,<, \times, 0,0,>, \times, \ldots
$$

where ... stand for empty positions and the left $\times$ is at position 0 , corresponds to the weight

$$
\lambda+\rho=(4,3,0 \mid 1,0,-4) .
$$

The translation functor $T(V)^{\chi, \tau}$ described in Lemma11 moves a simple module $L_{\lambda} \in \mathcal{F}^{\chi}$ to $L_{\mu} \in \mathcal{F}^{\tau}$ such that $f_{\mu}$ is obtained from $f_{\lambda}$ by moving a symbol $<$ or $>$ at position $t$ to the next right position $t+1$ or to the next left position $t-1$ (the position $t$ and the 
direction are determined by a choice of the core $\tau^{\prime}$ ). Assume that the chosen direction is to the right. If the next to the right position has 0 or $\times$, we exchange the symbols in position $t$ and $t+1$. For instance,

$$
\begin{gathered}
\ldots,<, 0, \ldots \longrightarrow \ldots, 0,<, \ldots \\
\ldots,<, \times, \ldots \longrightarrow \ldots, \times,<, \ldots
\end{gathered}
$$

The situation when the next to the right symbol is $<$ or $>$ is forbidden by the conditions on $\chi$ and $\tau$ (see Lemma 11). We move $<$ or $>$ to the left using the analogous rule.

Now for any dominant weight $\lambda$, let $\bar{\lambda}$ be the corresponding weight in the equivalent most atypical block of $\mathfrak{g}_{\chi}$. Then $f_{\bar{\lambda}}$ is obtained from $f_{\lambda}$ by moving all symbols $<,>$ to the right of all crosses by the procedures described above and then replacing all of them by 0 . In our example $f_{\bar{\lambda}}$ is

$$
\ldots, \times, 0,0, \times, \ldots
$$

with left $\times$ at position -1 .

Note also that shifting a weight diagram by one one position to the right corresponds to tensoring the corresponding module with the one dimensional representation of weight $(1, \ldots, 1 \mid-1, \ldots,-1)$.

Now let $\mathfrak{g}=\mathfrak{o s p}(2 m, 2 n)$. Set $\mathbb{T}=\mathbb{Z}_{\geq 0}$. For a dominant weight $\lambda$ such that $\lambda+\rho=$ $a_{1} \varepsilon_{1}+\ldots+a_{m} \varepsilon_{m}+b_{1} \delta_{1} \ldots+b_{n} \delta_{n}$ let

$$
X_{\lambda}=\left(\left|a_{1}\right|, \ldots,\left|a_{m}\right|\right), Y_{\lambda}=\left(b_{1}, \ldots, b_{n}\right), f_{\lambda}=f_{X_{\lambda}, Y_{\lambda}} .
$$

It is not difficult to see that $f_{\lambda}$ is a weight diagram of a dominant $\lambda$ if and only if

- for any $t \neq 0, f_{\lambda}(t)$ is empty or just one element set;

- the multiset $f_{\lambda}(0)$ does not contain $<$, contains $>$ with multiplicity at most 1 (it can contain any number of $x)$.

For example, if $\lambda=(2,0,0 \mid 3,0)$, then $f_{\lambda}=>x, 0,>,<, \ldots$ However, in this situation a weight is not uniquely determined by its weight diagram. More precisely, if $f(0) \neq 0$, there is exactly one weight with the weight diagram $f$, since all the coordinates of such a weight are non-negative. If $f(0)=0$, then the coordinate $a$ corresponding to the first $>$ or $\times$ can be chosen positive or negative. For instance if

$$
f=0,0,<, \times,>, \ldots,
$$

then the two weights $(4,3 \mid 3,2)$ and $(4,-3 \mid 3,2)$ are dominant and have $f$ as their weight diagram.

Terminology - To differenciate those two weights we call a dominant weight positive if it does not have negative coordinates, and negative otherwise.

The core of a weight can be obtained by replacing by 0 all $\times$ in the diagram. The translation functors from Lemma 11 can be described in the same way as in previous case, except that we do not allow a symbol to move from or to the zero position. Indeed, if we want to move $>$ from the zero position, we can get two weights corresponding to the same diagram, which means that the translation functor does not provide an equivalence of blocks. Thus, in this case we have two types of blocks, one with zero mark at its core ( $>$ at the zero position), and another without it. The former case corresponds to $\mathfrak{g}_{\chi}=\mathfrak{o s p}(2 k+2,2 k)$, the latter corresponds to $\mathfrak{g}_{\chi}=\mathfrak{o} \mathfrak{s p}(2 k, 2 k)$. Note that the atypicality degree $k$, as before, is the number of $\times$ in a weight diagram. Finally, to get the weight $\bar{\lambda}$ corresponding to $\lambda$ in the most atypical block, as in the $\mathfrak{g l}$ case, we move all $<,>$ to the right of all $\times$ (except one at zero position $)$ and then replace them by 0 . A positive 
weight goes to a positive one, and a negative weight goes to a negative one under this correspondence.

Below are two examples:

if $f_{\lambda}=0,0,<, \times,>, \times, \ldots$, then $f_{\bar{\lambda}}=0,0, \times, \times, \ldots$;

if $f_{\lambda}=<, 0,>,<, \ldots$, then $f_{\bar{\lambda}}={ }_{x}, 0, \ldots$

Now let us discuss the case $\mathfrak{o s p}(2 m+1,2 n)$. We assume that $\lambda$ is dominant and atypical, then all coordinates $a_{i}, b_{j}$ of $\lambda+\rho$ belong to $-\frac{1}{2}+\mathbb{Z}_{\geq 0}$. Let $\mathbb{T}=\frac{1}{2}+\mathbb{Z}_{\geq 0}$ and define $X_{\lambda}$, $Y_{\lambda}$ and $f_{\lambda}$ as in the case $\mathfrak{g}=\mathfrak{o s p}(2 m, 2 n)$. The dominance condition is equivalent to the following condition on a weight diagram $f$

- $f(t)$ is empty or one element set for any $t \neq \frac{1}{2}$;

- $f\left(\frac{1}{2}\right)$ may contain at most one of $<$ or $>$ and any number of $\times$.

As in the previous case, it is possible that two dominant weights have the same weight diagram. That may happen if $f\left(\frac{1}{2}\right)$ does not contain $>$ or $<$ and has at least one $\times$. For example, the diagram with two $\times$ at $\frac{1}{2}$ corresponds to $\left(\frac{1}{2},-\frac{1}{2} \mid \frac{1}{2}, \frac{1}{2}\right)$ and to $\left(-\frac{1}{2},-\frac{1}{2} \mid \frac{1}{2}, \frac{1}{2}\right)$. The translation functors, unlike in the previous case, mix those two types of weights. So if the weight diagram has at least one $\times$ and no $<,>$ at the position $\frac{1}{2}$ we put an indicator (which we sometimes refer to as "sign") \pm before the weight diagram in parentheses. Its value is + if the corresponding weight has the form

$$
\lambda+\rho=\left(a_{1}, \ldots, a_{m-s}, \frac{1}{2},-\frac{1}{2}, \ldots,-\frac{1}{2} \mid b_{1}, \ldots, b_{n}\right),
$$

and - if the corresponding weight has the form

$$
\lambda+\rho=\left(a_{1}, \ldots, a_{m-s},-\frac{1}{2},-\frac{1}{2}, \ldots,-\frac{1}{2} \mid b_{1}, \ldots, b_{n}\right),
$$

where $s$ is the number of crosses at the position $\frac{1}{2}$.

The translation functors of Lemma11 act on the diagrams, as in the previous case. The only difference is that one allows to move $<$ or $>$ from the position $\frac{1}{2}$ to the right but such move transforms a diagram without indicator to one with it. If $f\left(\frac{3}{2}\right)=0$, then the indicator of the new diagram is - , if $f\left(\frac{3}{2}\right)=\times$, then the indicator is + . For example

$$
\begin{aligned}
& \stackrel{<}{\times}, 0, \ldots \longrightarrow(-) \times,<, \ldots \\
& \stackrel{<}{\times}, \times, \ldots \longrightarrow(+)_{\times}^{\times},<, \ldots
\end{aligned}
$$

Moving $<$ (resp. $>$ ) at the position $\frac{3}{2}$ to the left is possible if $f\left(\frac{1}{2}\right)$ does not have $<$ or $>$ already, and therefore either $f\left(\frac{1}{2}\right)=0$ or $f\left(\frac{1}{2}\right)$ must have an indicator. If the indicator of $f$ is - we just move $<$ (resp. $>$ ) to the position $\frac{1}{2}$ and put $f\left(\frac{3}{2}\right)=0$. If the indicator of $f$ is + , one should exchange $<\left(\right.$ resp. $>$ ) at the position $\frac{3}{2}$ with one $\times$ at the position $\frac{1}{2}$. For example,

$$
\begin{aligned}
& (-) \times,<, 0, \ldots \longrightarrow \times \times<, \ldots, \\
& (+) \times,<, 0, \ldots \longrightarrow<, \times, \ldots
\end{aligned}
$$

To get the weight $\bar{\lambda}$ in the most atypical block corresponding to $\lambda$, one does the same as in two previous cases (one moves all $<$ and $>$ to the right of all crosses and then replaces them by 0$)$.

Remark - It is clear from above that the most atypical blocks with trivial central characters of Lie superalgebras $\mathfrak{o s p}(2 k+1,2 k+2)$ and $\mathfrak{o s p}(2 k+1,2 k)$ are equivalent. 


\section{Reduction to the most atypical Case}

Lemma 13. - Let $\alpha$ be a simple root of $\mathfrak{b}_{0}$ such that either $\alpha$ is a simple root of $\mathfrak{b}$ or $\alpha$ is a sum of two isotropic simple roots $\alpha_{1}+\alpha_{2}$. Let $\mathfrak{q}$ be the parabolic subalgebra with Levi part $\mathfrak{l}$ containing $\mathfrak{b}$ such that $\alpha$, respectively $\alpha_{1}, \alpha_{2}$ are orthogonal to all roots in $\Delta(\mathfrak{l})$. Assume that $\nu$ is a ld-dominant weight, such that $(\nu+\rho, \alpha)=0$ (and $\left(\nu+\rho, \alpha_{j}\right) \neq 0$ at least for one $j$ in the second case). Then $\Gamma_{i}\left(G / Q, L_{\nu}(\mathfrak{q})\right)=0$ for all $i \geq 0$.

Proof. - Consider the parabolic subalgebra $\mathfrak{p}$ obtained from $\mathfrak{q}$ by adding the roots $-\alpha$, $\left(-\alpha_{1},-\alpha_{2}\right.$ in the second case). The fibres of the canonical projection $\pi: G / P \rightarrow G / Q$ are isomorphic to $G^{\prime} / B^{\prime}$, where $\mathfrak{g}^{\prime}$ is isomorphic $\mathfrak{s l}(2)$ in the first case and $\mathfrak{s l}(1,2)$ in the second case, $\mathfrak{b}^{\prime}=\mathfrak{g}^{\prime} \cap \mathfrak{b}$. We claim that $\mathcal{L}_{\nu}(\mathfrak{p})_{\mid \text {fibre }}^{*}$ is acyclic. Indeed, in the first case $\mathcal{L}_{\nu}(\mathfrak{p})_{\mid \text {fibre }}^{*}$ is an invertible sheaf on $\mathbb{P}^{1}$, and the condition $(\nu+\rho, \alpha)=0$ immediately implies that it is acyclic. In the second case the underlying variety $\left(G^{\prime} / B^{\prime}\right)_{0}$ is isomorphic to $\mathbb{P}^{1}$. To calculate the cohomology we use Penkov's remark. The sheaf $\tilde{\mathcal{L}}_{\nu}(\mathfrak{p})_{\mid \text {fibre }}^{*}$ has a filtration with four simple terms, one dominant $\mathcal{O}_{\nu}$, two acyclic terms $\mathcal{O}_{\nu-\alpha_{1}}, \mathcal{O}_{\nu-\alpha_{2}}$, and one antidominant $\mathcal{O}_{\nu-\alpha_{1}-\alpha_{2}}$. If the cohomology groups of $\mathcal{L}_{\nu}(\mathfrak{p})_{\mid \text {fibre }}^{*}$ are non-trivial, they must be one-dimensional. But that would imply $\left(\nu+\rho, \alpha_{1}\right)=\left(\nu+\rho, \alpha_{2}\right)=0$. Contradiction.

Now the statement follows from Leray spectral sequence.

Lemma 14. - Let $\mathfrak{g}=\mathfrak{g l}(m, n)$. Let $\lambda$ be a dominant weight and $\chi=\chi_{\lambda}$. Let $V, \delta$ and $\tau$ satisfy all the conditions of Lemma 11, $T(V)^{\chi, \tau}\left(L_{\lambda}\right)=L_{\mu}$. Then

$$
\Gamma_{i}\left(G / B, L_{\mu}(\mathfrak{b})\right)=T(V)^{\chi, \tau}\left(\Gamma_{i}\left(G / B, L_{\lambda}(\mathfrak{b})\right)\right) .
$$

Proof. - By Corollary 1, one has

$$
\Gamma_{i}\left(G / B,\left(V \otimes L_{\lambda}(\mathfrak{b})\right)^{\Phi^{-1}(\tau)}\right)=T(V)^{\chi, \tau}\left(\Gamma_{i}\left(G / B, L_{\lambda}(\mathfrak{b})\right)\right) .
$$

We note that $\left(V \otimes L_{\lambda}(\mathfrak{b})\right)^{\Phi^{-1}(\tau)}$ has a filtration with simple quotients $L_{\nu}(\mathfrak{b})$ such that all $\nu \neq \mu$ are not dominant and satisfy the conditions of Lemma 13. Hence $\Gamma_{i}\left(G / B, L_{\nu}(\mathfrak{b})\right)=$ 0 for all $\nu \neq \mu$. The statement follows.

Corollary 4. - Let $\mathfrak{g}=\mathfrak{g l}(m, n)$. Let $\lambda$ be a dominant weight with atypicality degree $k$, $T$ be the functor which establishes an equivalence between $\mathcal{F}^{\chi_{\lambda}}$ and the most atypical block of $\mathfrak{g}_{\chi}$. Denote by $\bar{\lambda}$ the highest weight of $T\left(L_{\lambda}\right)$. If $B_{\chi}=B \cap G_{\chi}$, then

$$
K_{G, B}^{\lambda, \mu}(z)=K_{G_{\chi}, B_{\chi}}^{\bar{\lambda}, \bar{\mu}}(z)
$$

Proof. - Due to Lemma 14 it is sufficient to prove the statement for the case of stable $\lambda$ (see Lemma 8). Let $\mathfrak{p}=\mathfrak{g}_{\chi}+\mathfrak{b}$. Consider the natural projection $\pi: G / B \longrightarrow G / P$, the fibres of $\pi$ are isomorphic to $P / B=G_{\chi} / B_{\chi}$. We note that all the simple subquotients in $R^{q} \pi^{*}\left(\mathcal{L}_{\lambda}(\mathfrak{b})_{\mid \text {fibre }}^{*}\right)$ are stable, hence $H^{i}\left(G / P, R^{q} \pi^{*}\left(\mathcal{L}_{\lambda}(\mathfrak{b})_{\mid \text {fibre }}^{*}\right)\right)=0$ for $i>0$. The statement follows from Leray spectral sequence.

If $\mathfrak{g}=\mathfrak{g l}(m, n)$, the above corollary reduces the calculation of the cohomology groups of an invertible sheaf $\mathcal{O}_{\lambda}$ on the flag variety $G / B$ (with dominant $\lambda$ ) to the situation where $G=G_{\chi}$ and $\lambda$ has the same central character as the trivial module. If $\mathfrak{g}=\mathfrak{g l}(m, n)$ it is known that $\Gamma_{i}\left(G / B, L_{\lambda}(\mathfrak{b})\right)=0$ for all $i>0$, and $\Gamma_{0}\left(G / B, L_{\lambda}(\mathfrak{b})\right)$ is the Kac module $K_{\lambda}$. Hence, calculating $K_{G, B}^{\lambda, \mu}(z)={ }^{0} K_{G, B}^{\lambda, \mu}$ provides an algorithm for finding the characters of simple modules. That was done in [24] and we do not repeat these calculations here. 
Instead we concentrate on the case of the orthosymplectic group.

To do so, we have to use Theorem 11, and unfortunately we are only able to calculate the Euler characteristic $K_{G, B}^{\lambda, \mu}(-1)$. That would be sufficient to find the characters if we had an assertion like " $K_{G, B}^{\lambda, \lambda}(-1)=1$ and $K_{G, B}^{\lambda, \mu}(-1) \neq 0$ implies $\mu \leq \lambda "$.

However, in general this is not true, moreover, in some cases $K_{G, B}^{\lambda, \mu}(-1)=0$. So we should substitute $B$ by some larger parabolic subgroup $Q_{\lambda}$ which depends on $\lambda$.

Let $\lambda$ be a dominant weight. Denote by $\mathfrak{g}_{\lambda}$ the subalgebra of $\mathfrak{g}$ defined by the Dynkin subdiagram corresponding to the simple roots of $\mathfrak{g}$ such that all the coordinates of $\lambda$ restricted to $\mathfrak{g}_{\lambda}$ are zero and $\mathfrak{g}_{\lambda}$ is isomorphic to $\mathfrak{o s p}(2 s, 2 s), \mathfrak{o s p}(2 s+2,2 s)$ or $\mathfrak{o s p}(2 s+1,2 s)$.

Terminology - We call this subalgebra $\mathfrak{g}_{\lambda}$ the tail subalgebra of the weight $\lambda$ and the module $L_{\lambda}$, and call $s$ the length of the tail of $\lambda$.

The reader can check that $s$ is the number of $\times$ 's at 0 (respectively $\frac{1}{2}$ ) in the weight diagram except the case when the indicator has + . In the latter case $s$ is the number of $\times$ at $\frac{1}{2}$ minus 1. Let $\mathfrak{q}_{\lambda}$ be the parabolic subalgebra with Levi part $\mathfrak{g}_{\lambda}$.

Lemma 15. - Let $\lambda$ be a dominant weight and $\chi=\chi_{\lambda}$. Let $V, \delta$ and $\tau$ satisfy all the conditions of Lemma 11. Let $T(V)^{\chi, \tau}\left(L_{\lambda}\right)=L_{\mu}$. Then $Q_{\lambda}=Q_{\mu}$ and

$$
\Gamma_{i}\left(G / Q_{\lambda}, L_{\mu}\left(\mathfrak{q}_{\lambda}\right)\right)=T(V)^{\chi, \tau}\left(\Gamma_{i}\left(G / Q_{\lambda}, L_{\lambda}\left(\mathfrak{q}_{\lambda}\right)\right)\right) .
$$

Proof. - The same as of Lemma 14.

Lemma 16. - The functor $T$ from $\mathcal{F}^{\chi}$ to the most atypical block of $\mathfrak{g}_{\chi}$ containing trivial module, which provides an equivalence of categories, preserves the tails of simple modules.

Proof. - Straightforward.

For a dominant weight $\lambda$ let $\bar{\lambda}$ be the weight of $T\left(L_{\lambda}\right)$. Let $\mathfrak{q}_{\lambda}$ be the parabolic subalgebra of $\mathfrak{g}$ whose Levi part is $\mathfrak{h}+\mathfrak{g}_{\lambda}$. The following corollary can be proved exactly as Corollary 4 .

Corollary 5. - Let $\lambda$ be a dominant weight with central character $\chi$. Then

$$
K_{G, Q_{\lambda}}^{\lambda, \mu}(z)=K_{G_{\chi}, Q_{\bar{\lambda}}}^{\bar{\lambda}, \bar{\mu}}(z) .
$$

The above corollary reduces the calculation of $K_{G, Q_{\lambda}}^{\lambda, \mu}(-1)$ to the case where $\mathfrak{g}$ is one of $\mathfrak{o s p}(2 k, 2 k), \mathfrak{o s p}(2 k+2,2 k), \mathfrak{o s p}(2 k+1,2 k)$, and $\lambda$ and $\mu$ have the trivial central character.

\section{RECURSION}

In this section we assume that $\mathfrak{g}$ is either $\mathfrak{o s p}(2 k, 2 k), \mathfrak{o s p}(2 k+2,2 k)$ or $\mathfrak{o} \mathfrak{s p}(2 k+1,2 k)$. Then all the simple roots are odd and the Dynkin diagram of $\mathfrak{g}$ is either

$$
\otimes-\ldots-\otimes_{\otimes}^{\otimes} \mid
$$

(with $2 k$ vertices if $\mathfrak{g}=\mathfrak{o s p}(2 k, 2 k)$ and $2 k+1$ vertices if $\mathfrak{g}=\mathfrak{o s p}(2 k+2,2 k)$ ) or

$$
\otimes-\ldots-\otimes \rightarrow \bullet
$$

(with $2 k$ vertices for $\mathfrak{g}=\mathfrak{o s p}(2 k+1,2 k)$ ). The corresponding Borel subalgebra is called mixed (14).

Let $\mathfrak{s}^{1}$ be the subalgebra of $\mathfrak{g}$ generated by all the simple roots of $\mathfrak{g}$ except the first two, $\mathfrak{s}^{2}$ be the subalgebra of $\mathfrak{s}^{1}$ generated by all the simple roots of $\mathfrak{s}^{1}$ except the first two 
etc... Each time $\mathfrak{s}^{i}$ has the same type as $\mathfrak{g}$. Let $\mathfrak{p}^{i}$ be the parabolic subalgebra with Levi subalgebra $\mathfrak{l}^{i}=\mathfrak{h}+\mathfrak{s}^{i}$ for $i \leq k-1$, and let $\mathfrak{p}^{k}=\mathfrak{b}$. We have a flag of parabolic subalgebras

$$
\mathfrak{g} \supset \mathfrak{p}^{1} \supset \cdots \supset \mathfrak{p}^{k}=\mathfrak{b} .
$$

In this section, we assume that the simple finite-dimensional module $L_{\lambda}$ has the same central character as the trivial module, and we denote this central character by $\chi$. In the case of $\mathfrak{o s p}(2 k, 2 k)$, we also assume that $\lambda$ is positive. That implies

$$
\lambda+\rho=a_{1} \varepsilon_{1}+\ldots+a_{k} \varepsilon_{k}+b_{1} \delta_{1}+\ldots+b_{k} \delta_{k},
$$

Moreover, $\left|a_{i}\right|=\left|b_{i}\right|$ for all $i \leq k$. The weight diagram of $\lambda$ does not have symbols $>,<$ except $>$ at 0 for $\mathfrak{g}=\mathfrak{o s p}(2 k+2,2 k)$.

Remark - Let $\mathfrak{g}=\mathfrak{o s p}(2 k, 2 k)$ and

$$
\lambda=a_{1}\left(\varepsilon_{1}+\delta_{1}\right)+\ldots+a_{k}\left(\varepsilon_{k}+\delta_{k}\right),
$$

define

$$
\lambda^{\prime}=a_{1}\left(\varepsilon_{1}+\delta_{1}\right)+\ldots+a_{k}\left(-\varepsilon_{k}+\delta_{k}\right) .
$$

If $\sigma$ is the automorphism of $\mathfrak{g}$ induced by the symmetry of the Dynkin diagram, and $M^{\sigma}$ is the module obtained from $M$ by twisting by $\sigma$, then $L_{\lambda}^{\sigma}=L_{\lambda^{\prime}}$. Since $\sigma$ acts on $G / P^{1}$, one has

$$
K_{G, P^{1}}^{\lambda, \mu}(z)=K_{G, P^{1}}^{\lambda^{\prime}, \mu^{\prime}}(z)
$$

Thus, if we know $K_{G, P^{1}}^{\lambda, \mu}(z)$ for all positive $\lambda$, we can easily obtain them for all $\lambda$.

In this section, we give a recursion procedure to compute the polynomials $K_{G, P^{1}}^{\lambda, \mu}(z)$. This recursion is double: if $\lambda$ is "far from tail and far from the even walls" (Proposition 2), we get it "closer to the tail and the walls". If $\lambda$ is close to the wall and far from the tail (Proposition 3) we decrease the rank of the Lie superalgebra. Finally, when $\lambda$ is very close to the tail, we compute the cohomology directly (Propositions 4, 5] in the next section).

For a Laurent polynomial $F(z) \in \mathbb{C}\left[z, z^{-1}\right]$, we denote by $F(z)_{+}$the polynomial obtained from $F(z)$ by removing the monomials with negative powers of $z$.

Notation - Let $\alpha=\varepsilon_{1}+\delta_{1}$.

Before we start, we prove several technical statements that will be needed later.

Recall that $E$ denotes the standard $\mathfrak{g}$-module.

Lemma 17. - Let $\tau$ be a dominant central character with degree of atypicality $k-1$. Then $\left(L_{\lambda} \otimes E\right)^{\tau}$ is either simple or zero.

Let $\beta=\varepsilon_{i}+\delta_{i}, \lambda-\beta$ is dominant and $a_{i}>\frac{3}{2}$. Let $\tau=\chi_{\lambda-\delta_{i}}$ or $\chi_{\lambda-\varepsilon_{i}}$. Then $\left(L_{\lambda} \otimes E\right)^{\tau}=0$.

Proof. - There exists at most one weight $\gamma$ of $E$ such that $\lambda+\gamma$ is dominant and $\chi_{\lambda+\gamma}=\tau$. The best way to see it is via weight diagrams. Indeed, the weight diagram of $\lambda+\gamma$ is obtained from that of $\lambda$ by "separating" one $\times$ in two halves $>,<$ and moving one half one position to the left or to the right. It is clear that in this way one can get at most one dominant weight diagram with given core. Hence the first statement.

To prove the second statement, assume the opposite, say, $\left(L_{\lambda} \otimes E\right)^{\chi}=L_{\lambda-\delta_{i}}$. Let $M_{\pi}$ denote the Verma module with highest weight $\pi$. Then $\left(M_{\lambda} \otimes E\right)^{\tau}$ has a filtration by Verma modules with highest weights $\lambda-\gamma$ for all weights $\gamma$ of $E$ such that $\chi_{\lambda-\gamma}=\tau$. By direct inspection, $\lambda-\delta_{i}$ is the only such weight. Therefore $\left(M_{\lambda} \otimes E\right)^{\tau}=M_{\lambda-\delta_{i}}$. Similarly, 
$\left(M_{\lambda-\beta} \otimes E\right)^{\tau}$ has a filtration by Verma modules, one of the terms of this filtration is $M_{\lambda-\delta_{i}}$. Thus, $L_{\lambda-\delta_{i}}$ occurs in $\left(M_{\lambda-\beta} \otimes E\right)^{\tau}$.

It is known (one can find a proof in [1]) that

$$
\operatorname{Hom}_{\mathfrak{g}}\left(M_{\lambda-\beta}, M_{\lambda}\right) \neq 0 .
$$

Consider the exact sequence

$$
0 \rightarrow S \rightarrow M_{\lambda-\beta} \rightarrow M_{\lambda} \rightarrow F \rightarrow 0 .
$$

Apply the translation functor $T(E)^{\chi, \tau}$ to it

$$
0 \rightarrow(S \otimes E)^{\tau} \rightarrow\left(M_{\lambda-\beta} \otimes E\right)^{\tau} \rightarrow M_{\lambda-\delta_{i}} \rightarrow(F \otimes E)^{\tau} \rightarrow 0 .
$$

Since all weights of $S$ are strictly less than $\lambda-\beta, \lambda-\delta_{i}$ is not a weight of $(S \otimes E)^{\chi}$, hence the latter does not have a simple component $L_{\lambda-\delta_{i}}$. Therefore, by above, the multiplicity of $L_{\lambda-\delta_{i}}$ in $(F \otimes E)^{\tau}$ is zero. But $(F \otimes E)^{\tau}$ is a highest weight module with highest weight $\lambda-\delta_{i}$. Therefore $(F \otimes E)^{\tau}=0$. Since $L_{\lambda}$ is a quotient of $F$, we get $\left(L_{\lambda} \otimes E\right)^{\tau}=0$.

Lemma is proven.

The following lemma is very important in our calculations. We will use it in induction step to reduce the rank of $\mathfrak{g}$.

Lemma 18. - Let $\tau \neq \chi$ be a dominant central character with atypicality degree $k$ or $k-1$, $\kappa$ be a dominant weight with central character $\tau$ (see section 5 for definition). Assume that $(\kappa+\rho, \alpha)=0$. Let $T$ be the functor establishing an equivalence between $\mathcal{F}^{\tau}$ and the maximal atypical block of $\mathfrak{g}_{\tau}$. Let $T\left(L_{\mu}\right)=L_{\bar{\mu}}\left(\mathfrak{g}_{\tau}\right)$. Then

$$
K_{G, P^{1}}^{\kappa, \mu}(z)=K_{G_{\tau}, P_{\tau}^{1}}^{\bar{\kappa}, \bar{p}}(z),
$$

where $P_{\tau}^{1} \subset G_{\tau}$ is the analogue of $P^{1}$ for $G_{\tau}$.

Proof. - The weight diagram $f_{\kappa}$ has $\times$ at the rightmost non-empty position, and symbols $<$ and $>$ somewhere to the left of this $\times$. Using translation functors one can move those symbols next to the rightmost $\times$. (If one of those $<,>$ is at position 0 we don't move it, just move the second symbol.) Those translation functors commute with $\Gamma_{i}\left(G / P^{1}, \bullet\right)$ as their action on simple $\mathfrak{p}^{1}$-modules is the same as on the corresponding $\mathfrak{g}$-modules. Hence we can assume without loss of generality that $<$ and $>$ stand next to the rightmost $\times$ of $\kappa$. Let $\mathfrak{q}=\mathfrak{p}_{\tau}^{1}+\mathfrak{b}$. We claim that, in this case, $\Gamma_{i}\left(G / P^{1}, L_{\kappa}\left(\mathfrak{p}^{1}\right)\right)=\Gamma_{i}\left(G / Q, L_{\kappa}(\mathfrak{q})\right)$. Indeed, this follows from the fact that the restriction of $\kappa$ on $\mathfrak{g}_{\tau}$ is typical with respect to $\mathfrak{p}_{\tau}^{1}=\mathfrak{q} \cap \mathfrak{g}_{\tau}$, and by the typical lemma, the Leray spectral sequence for the canonical projection $\pi: G / Q \longrightarrow G / P^{1}$ degenerates, $\left(R^{q} \pi^{*} \mathcal{L}_{\kappa}^{*}=0\right.$ for all $\left.q>0\right)$. Now consider the translation functor which moves $<$ and $>$ to the right of the rightmost $\times$. This functor commutes with $\Gamma_{i}(G / Q, \bullet)$, since it moves a simple $\mathfrak{q}$-module to the one whose simple subquotients are all acyclic except one (as in the proof of Lemma 14). To finish the proof use stability of the weight obtained from $\kappa$ by translations and proceed as in the proof of Corollary 4 .

Let $\mathfrak{g}=\mathfrak{o s p}(2 k+1,2 k)$. Let $\lambda^{\prime}=\lambda$ if the diagram of $\lambda$ does not have a sign. If the weight diagram of $\lambda$ has a sign let $\lambda^{\prime}$ denote the weight whose diagram is obtained from that of $\lambda$ by switching the sign. For example, if $\lambda=0$, then $\lambda^{\prime}=\varepsilon_{1}$.

Recall that $\Phi$ is defined just before Lemma 4 .

Lemma 19. - Let $\mathfrak{g}=\mathfrak{o s p}(2 k+1,2 k)$. Then $T(E)^{\chi, \chi}$ is an equivalence of categories, and $T(E)^{\chi, \chi}\left(L_{\lambda}\right)=L_{\lambda^{\prime}}$. 
Proof. - The only dominant weights with central character $\chi$ of the form $\lambda+\gamma, \gamma$ being a weight of $E$, are $\lambda$ and $\lambda^{\prime}$. It suffices to show then that $T(E)^{\chi, \chi}\left(L_{\lambda}\right)=L_{\lambda^{\prime}}$ for $\lambda^{\prime} \neq \lambda$.

A direct calculation proves the statement for $E\left(\lambda=\varepsilon_{1}\right)$ and for the trivial module $(\lambda=0)$. Choose a maximal parabolic subalgebra $\mathfrak{p}$ with semi-simple part $\mathfrak{s}$ such that $L_{\lambda}(\mathfrak{s})$ is either standard or trivial. Then

$$
\left(L_{\lambda}(\mathfrak{p}) \otimes E\right)^{\Phi^{-1}(\chi)}=L_{\lambda^{\prime}}(\mathfrak{p}),
$$

and

$$
\left(\Gamma_{0}\left(G / P, L_{\lambda}(\mathfrak{p})\right) \otimes E\right)^{\chi}=\Gamma_{0}\left(G / P, L_{\lambda^{\prime}}(\mathfrak{p})\right) .
$$

Note that $\Gamma_{0}\left(G / P, L_{\lambda^{\prime}}(\mathfrak{p})\right)$ does not contain any subquotient isomorphic to $L_{\lambda}$, because the corresponding parabolically induced module does not have $L_{\lambda}$ as a subquotient. Therefore the exact sequence $\Gamma_{0}\left(G / P, L_{\lambda}(\mathfrak{p})\right) \rightarrow L_{\lambda} \rightarrow 0$, after application of $T(E)^{\chi, \chi}$, becomes $\Gamma_{0}\left(G / P, L_{\lambda^{\prime}}(\mathfrak{p})\right) \rightarrow L_{\lambda^{\prime}} \rightarrow 0$. That implies the statement.

We call $T(E)^{\chi, \chi}$ the switch functor. Lemma 1 and Lemma 19 imply that for any $\lambda \neq 0, \varepsilon_{1}$

$$
K_{G, P^{1}}^{\lambda, \mu}(z)=K_{G, P^{1}}^{\lambda^{\prime}, \mu^{\prime}}(z) .
$$

Proposition 2. - Let $a_{1}>\frac{3}{2}$ and $a_{1}>\left|a_{2}\right|+1$ if $k>1$. Then

i) $K_{G, P^{1}}^{\lambda, \mu}(z)=\left(z^{-1} K_{G, P^{1}}^{\lambda-\alpha, \mu}(z)\right)+$ for $\mu \notin\{\lambda, \lambda-\alpha\}$,

ii) $K_{G, P^{1}}^{\lambda, \lambda}(z)=1$

iii) $K_{G, P^{1}}^{\lambda, \lambda-\alpha}(z)=1$.

To prove the proposition we start with the following

Lemma 20. - Let $\lambda$ be as in the proposition, $\nu=\lambda-\varepsilon_{1}$. One has the following short exact sequence of $\mathfrak{p}^{1}$-modules:

$$
0 \rightarrow L_{\lambda}\left(\mathfrak{p}^{1}\right) \rightarrow\left(L_{\nu}\left(\mathfrak{p}^{1}\right) \otimes E\right)^{\Phi^{-1}(\chi)} \rightarrow L_{\lambda-\alpha}\left(\mathfrak{p}^{1}\right) \rightarrow 0 .
$$

Proof. - Consider the case $\mathfrak{g}=\mathfrak{o s p}(2 k+2,2 k)$ or $\mathfrak{o s p}(2 k+1,2 k)$. Note that $\mathfrak{p}^{1}$-irreducible subquotients of $E$ are $L_{ \pm \varepsilon_{1}}\left(\mathfrak{p}^{1}\right), L_{\varepsilon_{2}}\left(\mathfrak{p}^{1}\right)$ and $L_{ \pm \delta_{1}}\left(\mathfrak{p}^{1}\right)$. All of them except $L_{\varepsilon_{2}}\left(\mathfrak{p}^{1}\right)$ are one-dimensional. It is easy to check that the only $\mathfrak{p}^{1}$-dominant weights of the form $\nu+\gamma$ ( $\gamma$ being a weight of $E$ ) with central character $\chi$ are $\lambda$ and $\lambda-\alpha$. Hence we have

$$
\begin{gathered}
\left(L_{\nu}\left(\mathfrak{p}^{1}\right) \otimes L_{-\varepsilon_{1}}\left(\mathfrak{p}^{1}\right)\right)^{\Phi^{-1}(\chi)}=\left(L_{\nu}\left(\mathfrak{p}^{1}\right) \otimes L_{\varepsilon_{2}}\left(\mathfrak{p}^{1}\right)\right)^{\Phi^{-1}(\chi)}= \\
=\left(L_{\nu}\left(\mathfrak{p}^{1}\right) \otimes L_{\delta_{1}}\left(\mathfrak{p}^{1}\right)\right)^{\Phi^{-1}(\chi)}=0 .
\end{gathered}
$$

On the other hand,

$$
\begin{gathered}
L_{\nu}\left(\mathfrak{p}^{1}\right) \otimes L_{-\delta_{1}}\left(\mathfrak{p}^{1}\right)=L_{\lambda-\alpha}\left(\mathfrak{p}^{1}\right), \\
L_{\nu}\left(\mathfrak{p}^{1}\right) \otimes L_{\varepsilon_{1}}\left(\mathfrak{p}^{1}\right)=L_{\lambda}\left(\mathfrak{p}^{1}\right) .
\end{gathered}
$$

The exact sequence follows immediately.

The case $\mathfrak{g}=\mathfrak{o s p}(2 k, 2 k)$ can be done in the same way with the substitution of $\delta_{2}$ in place of $\varepsilon_{2}$.

The short exact sequence of Lemma 20 leads to the following long exact sequence:

$$
\begin{aligned}
\cdots \rightarrow \Gamma_{1}\left(G / P^{1}, L_{\lambda-\alpha}\left(\mathfrak{p}^{1}\right)\right) & \rightarrow \Gamma_{0}\left(G / P^{1}, L_{\lambda}\left(\mathfrak{p}^{1}\right)\right) \rightarrow \Gamma_{0}\left(G / P^{1},\left(L_{\nu}\left(\mathfrak{p}^{1}\right) \otimes E\right)^{\Phi^{-1}(\chi)}\right) \rightarrow \\
& \rightarrow \Gamma_{0}\left(G / P^{1}, L_{\lambda-\alpha}\left(\mathfrak{p}^{1}\right)\right) \rightarrow 0 .
\end{aligned}
$$


By Corollary 1 we have

$$
\Gamma_{i}\left(G / P^{1},\left(L_{\nu}\left(\mathfrak{p}^{1}\right) \otimes E\right)^{\Phi^{-1}(\chi)}\right)=\left(\Gamma_{i}\left(G / P^{1}, L_{\nu}\left(\mathfrak{p}^{1}\right)\right) \otimes E\right)^{\chi} .
$$

Now note that $\nu$ is $\mathfrak{p}^{1}$-typical. Therefore $\Gamma_{i}\left(G / P^{1},\left(L_{\nu}\left(\mathfrak{p}^{1}\right)\right)=0\right.$ for all $i>0$, and $\Gamma_{0}\left(G / P^{1},\left(L_{\nu}\left(\mathfrak{p}^{1}\right)\right)=L_{\nu}\right.$. Thus, the exact sequence degenerates into the following

$$
\Gamma_{i}\left(G / P^{1}, L_{\lambda-\alpha}\left(\mathfrak{p}^{1}\right)\right) \simeq \Gamma_{i-1}\left(G / P^{1}, L_{\lambda}\left(\mathfrak{p}^{1}\right)\right) \text { for } i \geq 2,
$$

and

$0 \rightarrow \Gamma_{1}\left(G / P^{1}, L_{\lambda-\alpha}\left(\mathfrak{p}^{1}\right)\right) \rightarrow \Gamma_{0}\left(G / P^{1}, L_{\lambda}\left(\mathfrak{p}^{1}\right)\right) \rightarrow\left(L_{\nu} \otimes E\right)^{\chi} \rightarrow \Gamma_{0}\left(G / P^{1}, L_{\lambda-\alpha}\left(\mathfrak{p}^{1}\right)\right) \rightarrow 0$.

Now we concentrate on the last exact sequence.

Denote by $V_{\lambda}$ the image of $\Gamma_{0}\left(G / P^{1}, L_{\lambda}\left(\mathfrak{p}^{1}\right)\right)$ in $\left(L_{\nu} \otimes E\right)^{\chi}$. Since $V_{\lambda}$ is isomorphic to a quotient of $\Gamma_{0}\left(G / P^{1}, L_{\lambda}\left(\mathfrak{p}^{1}\right)\right)$, Lemma 2 implies that $V_{\lambda}$ is generated by a highest vector of weight $\lambda$. Lemma 23 below explains the structure of $V_{\lambda}$.

Lemma 21. - The module $\left(L_{\nu} \otimes E\right)^{\chi}$ is a contragredient $\mathfrak{g}$-module with a unique irreducible submodule and a unique irreducible quotient isomorphic to $L_{\lambda-\alpha}$.

Proof. - The module $\left(L_{\nu} \otimes E\right)^{\chi}$ is contragredient because the tensor product of contragredient modules is contragredient and all the simple modules are contragredient. The only dominant weights of the form $\nu+\gamma(\gamma$ being a weight of $E)$ with central character $\chi$ are $\lambda$ and $\lambda-\alpha$. Hence they are the only possible weights of $\mathfrak{b}$-singular vectors in $\left(L_{\nu} \otimes E\right)^{\chi}$. The exact sequence 11 implies that $\left(L_{\nu} \otimes E\right)^{\chi}$ has simple subquotients isomorphic to $L_{\lambda}$ and $L_{\lambda-\alpha}$.

Assume that the socle of $\left(L_{\nu} \otimes E\right)^{\chi}$ contains a $\mathfrak{b}$-singular vector of weight $\lambda$. This gives an inclusion $L_{\lambda} \subset\left(L_{\nu} \otimes E\right)^{\chi}$ and, by duality, a surjection $\left(L_{\nu} \otimes E\right)^{\chi} \rightarrow L_{\lambda}$. Since there is only one vector of weight $\lambda$, the composition of those two maps must be the identity, thus the exact sequence 11 splits. Hence $\Gamma_{0}\left(G / P^{1}, L_{\lambda}\left(\mathfrak{p}^{1}\right)\right)$ is contragredient too, and since it's generated by a $\mathfrak{b}$-singular vector of weight $\lambda-\alpha$, it must be equal to $L_{\lambda-\alpha}$. Thus, if $\left(L_{\nu} \otimes E\right)^{\chi}$ contains $L_{\lambda}$, it is equal to $L_{\lambda} \oplus L_{\lambda-\alpha}$.

Let us show that this is impossible. Assume the opposite. Then

$$
\mathbb{C}=\operatorname{Hom}_{\mathfrak{g}}\left(L_{\lambda}, L_{\nu} \otimes E\right)=\operatorname{Hom}_{\mathfrak{g}}\left(L_{\lambda} \otimes E, L_{\nu}\right),
$$

hence $\left(L_{\lambda} \otimes E\right)^{\chi_{\nu}}=L_{\nu}$. But Lemma $17\left(\right.$ with $\left.\tau=\chi_{\nu}\right)$ implies $\left(L_{\lambda} \otimes E\right)^{\chi_{\nu}}=0$. Contradiction.

So now, we are sure that the socle of $\left(L_{\nu} \otimes E\right)^{\chi}$ is isomorphic to $L_{\lambda-\alpha}$; by duality, the same holds for the head, which is a quotient of $\Gamma_{0}\left(G / P^{1}, L_{\lambda}\left(\mathfrak{p}^{1}\right)\right)$.

Lemma 22. - The only weight $\mu$ such that $L_{\mu}$ may occur as a simple subquotient in both $\Gamma_{0}\left(G / P^{1}, L_{\lambda}\left(\mathfrak{p}^{1}\right)\right)$ and $\Gamma_{0}\left(G / P^{1}, L_{\lambda-\alpha}\left(\mathfrak{p}^{1}\right)\right)$ is $\lambda-\alpha$.

Proof. - We prove the lemma by induction on the dimension of $\mathfrak{g}$. Let $\mathfrak{g}$ be the smallest for which the statement is not true. Let $L_{\mu}$ occur in both $\Gamma_{0}\left(G / P^{1}, L_{\lambda}\left(\mathfrak{p}^{1}\right)\right)$ and $\Gamma_{0}\left(G / P^{1}, L_{\lambda-\alpha}\left(\mathfrak{p}^{1}\right)\right)$. Assume first that $\mu \neq 0$ if $\mathfrak{g}=\mathfrak{o s p}(2 k+1,2 k)$ or $\mathfrak{o s p}(2 k+2,2 k)$. Let $\omega$ be the highest weight of $E,\left(\omega=\varepsilon_{1}\right.$ if $\mathfrak{g}=\mathfrak{o s p}(2 k+1,2 k)$ or $\mathfrak{o s p}(2 k+2,2 k)$ and $\delta_{1}$ if $\left.\mathfrak{g}=\mathfrak{o s p}(2 k, 2 k)\right)$, and let $\tau=\chi_{\mu+\omega}$. Clearly $\tau$ satisfies the conditions of Lemma 18, and $\left(L_{\mu} \otimes E\right)^{\tau}=L_{\mu+\omega}$. Therefore, by Corollary 1, $L_{\mu+\omega}$ occurs in both $\Gamma_{0}\left(G / P^{1},\left(L_{\lambda}\left(\mathfrak{p}^{1}\right) \otimes E\right)^{\Phi^{-1}(\tau)}\right)$ and $\Gamma_{0}\left(G / P^{1},\left(L_{\lambda-\alpha}(\mathfrak{p}) \otimes E\right)^{\Phi^{-1}(\tau)}\right)$. 
Note that the first mark of $\mu$ is strictly less than that of $\lambda-\alpha$ by Lemma 2 , hence one can apply Lemma 17 to $\mathfrak{s}^{1}$ and get some dominant weights $\lambda_{1}$ and $\lambda_{2}$ such that

$$
\begin{gathered}
\left(L_{\lambda}\left(\mathfrak{p}^{1}\right) \otimes E\right)^{\Phi^{-1}(\tau)}=L_{\lambda_{1}}\left(\mathfrak{p}^{1}\right), \\
\left(L_{\lambda-\alpha}\left(\mathfrak{p}^{1}\right) \otimes E\right)^{\Phi^{-1}(\tau)}=L_{\lambda_{2}}\left(\mathfrak{p}^{1}\right) .
\end{gathered}
$$

Moreover, it is easy to check that $\lambda_{2}=\lambda_{1}-\alpha$. Therefore $K_{G, P^{1}}^{\lambda_{i}, \mu+\omega}(0) \neq 0$ for $i=1,2$.

By Lemma 18, $L \overline{\mu+\omega}$ occurs in both $\Gamma_{0}\left(G_{\tau} / P_{\tau}^{1}, L_{\bar{\lambda}_{1}}\left(\mathfrak{p}_{\tau}^{1}\right)\right)$ and $\Gamma_{0}\left(G_{\tau} / P_{\tau}^{1},\left(L_{\bar{\lambda}_{2}}\left(\mathfrak{p}_{\tau}^{1}\right)\right)\right.$. Note that $\bar{\lambda}_{2}=\bar{\lambda}_{1}-\alpha^{\prime}$, where $\alpha^{\prime}$ is the analogue of $\alpha$ for $\mathfrak{g}_{\tau}$. Thus, since the statement of the lemma is not true for $\mathfrak{g}_{\tau}$, that contradicts the minimality of $\mathfrak{g}$.

If $\mu=0$ and $\mathfrak{g}=\mathfrak{o s p}(2 k+1,2 k)$ or $\mathfrak{o s p}(2 k+2,2 k)$, we can not apply Lemma 17, In the case $\mathfrak{g}=\mathfrak{o s p}(2 k+1,2 k)$ one should use $\mu^{\prime}=\varepsilon_{1}$ and (9). If $\mathfrak{g}=\mathfrak{o s p}(2 k+2,2 k)$, we use the automorphism $\sigma$ induced by the symmetry of the Dynkin diagram. Note that $\sigma$ acts trivially on the modules with central character $\chi$ but switches some simple modules in the block with central character $\tau$. One gets

$$
\begin{gathered}
\left(L_{\lambda}\left(\mathfrak{p}^{1}\right) \otimes E\right)^{\Phi^{-1}(\tau)}=L_{\lambda_{1}}\left(\mathfrak{p}^{1}\right) \oplus L_{\lambda_{1}}^{\sigma}\left(\mathfrak{p}^{1}\right), \\
\left(L_{\lambda-\alpha}\left(\mathfrak{p}^{1}\right) \otimes E\right)^{\Phi^{-1}(\tau)}=L_{\lambda_{2}}\left(\mathfrak{p}^{1}\right) \oplus L_{\lambda_{2}}^{\sigma}\left(\mathfrak{p}^{1}\right)
\end{gathered}
$$

for some dominant weights $\lambda_{1}$ and $\lambda_{2}$ such that $\lambda_{2}=\lambda_{1}-\alpha$. Since $L_{\omega}$ is $\sigma$-invariant, it occurs in the image of the functor $\Gamma_{0}$ of all four summands, and one can finish the proof as in general case.

Lemma 23. - One has the exact sequence:

$$
0 \rightarrow L_{\lambda-\alpha} \rightarrow V_{\lambda} \rightarrow L_{\lambda} \rightarrow 0
$$

Proof. - Recall that we have the exact sequence

$$
0 \rightarrow V_{\lambda} \rightarrow\left(L_{\nu} \otimes E\right)^{\chi} \rightarrow \Gamma_{0}\left(G / P^{1}, L_{\lambda-\alpha}\left(\mathfrak{p}^{1}\right)\right) \rightarrow 0 .
$$

From Lemma 20 we know that $L_{\lambda-\alpha}$ is a submodule of $V_{\lambda}$ and $L_{\lambda}$ is the quotient of $V_{\lambda}$ by the unique maximal submodule $N$. Suppose that $N$ has another subquotient $L_{\mu}$ with $\mu \neq \lambda-\alpha$. Denote by $N^{\prime}$ the orthogonal complement to $N$ with respect to the contravariant form on $\left(L_{\nu} \otimes E\right)^{\chi}$. Since $N^{\prime}$ has a simple subquotient $L_{\lambda}$, we have $V_{\lambda} \subset N^{\prime}$. But $\left(L_{\mu} \otimes E\right)^{\chi} / N^{\prime}$ is isomorphic to the module contragredient to $N$, hence it must have a subquotient $L_{\mu}$. Thus, we obtain that $L_{\mu}$ is a subquotient in both $\Gamma_{0}\left(G / P^{1}, L_{\lambda}\left(\mathfrak{p}^{1}\right)\right)$ and $\Gamma_{0}\left(G / P^{1}, L_{\lambda-\alpha}\left(\mathfrak{p}^{1}\right)\right)$. That contradicts Lemma 22,

The identity (10), the exact sequence (11) and Lemma 23 imply Proposition 2.

Terminology - A pair of weights $(\lambda, \mu)$ is called exceptional, if $K_{G, P^{1}}^{\lambda, \mu}(z) \neq 0$ and the first coordinate of $\mu$ is less than the second coordinate of $\lambda$.

Proposition 3. - Assume $k \geq 2, \lambda$ is such that $a_{1}=a_{2}+1$, and $a_{2} \neq \frac{1}{2},-\frac{1}{2}, 0$. Assume that $(\lambda, \mu)$ is not an exceptional pair. One has:

i) $K_{G, P^{1}}^{\lambda, \mu}(z)=z K_{P^{1}, P^{2}}^{\lambda-\alpha)}(z)$ if $\mu \neq \lambda, \lambda-\alpha$;

ii) $K_{G, P^{1}}^{\lambda, \lambda}(z)=1$;

iii) $K_{G, P^{1}}^{\lambda, \lambda-\alpha}(z)=0$. 
Let

$$
\begin{gathered}
\nu=\lambda-\delta_{1} \text { for } \mathfrak{g}=\mathfrak{o s p}(2 k+1,2 k) \text { or } \mathfrak{o s p}(2 k+2,2 k) \\
\nu=\lambda-\varepsilon_{1} \text { for } \mathfrak{g}=\mathfrak{o s p}(2 k, 2 k) .
\end{gathered}
$$

Note that $\nu$ is not dominant but is $\mathfrak{p}^{1}$-dominant. Observe also that $\nu$ satisfies the conditions of Lemma 13 for $\mathfrak{q}=\mathfrak{p}^{2}$. Therefore

$$
\Gamma_{i}\left(G / P^{2}, \mathcal{L}_{\nu}\left(\mathfrak{p}^{2}\right)\right)=0 \text { for all } i \geq 0 .
$$

Lemma 24. - One has $K_{G, P^{1}}^{\nu, \mu}(z)=z K_{P^{1}, P^{2}}^{\nu, \mu}(z)$ for any $\mu \neq \nu$, and $K_{G, P^{1}}^{\nu, \nu}(z)=0$.

Proof. - The notations are adapted from those of the proof of Theorem 1, Let $\pi$ denote the canonical projection $\pi: G / P^{2} \rightarrow G / P^{1}$. The derived functors $\left(R^{q} \pi_{*} \mathcal{L}_{\nu}\left(\mathfrak{p}^{2}\right)^{*}\right)$ have the following property which can be easily obtained from Lemma 3 applied to $\Gamma_{i}\left(P^{1} / P^{2}, L_{\nu}\left(\mathfrak{p}^{2}\right)\right)$ :

- the second coordinate of $\mu+\rho$ for any $\mu \neq \nu$ such that $L_{\mu}$ occurs in $\left(R^{q} \pi_{*} \mathcal{L}_{\nu}\left(\mathfrak{p}^{2}\right)^{*}\right)$ is strictly less than $a_{1}-1$, hence any such $\mu$ is $\mathfrak{p}^{1}$-typical;

- $L_{\nu}$ only occurs in $\left(R^{0} \pi_{*} \mathcal{L}_{\nu}\left(\mathfrak{p}^{2}\right)^{*}\right)$.

Hence the second terms of the Leray spectral sequence are all zero except $E_{2}^{p, 0}$ and $E_{2}^{0, q}$ (for any $p, q$ ): it is just the typical Lemma 5 .

Now the identity (12) asserts that this spectral sequence converges to zero. So the conclusion is that there is an isomorphism between $E_{2}^{p, 0}$ and $E_{2}^{0, p-1}$ and $E_{2}^{0,0}=0$ (it can't come from anybody and in the end it should be zero). So

$$
H^{p}\left(G / P^{1}, \mathcal{L}_{\nu}\left(\mathfrak{p}^{1}\right)^{*}\right) \simeq H^{0}\left(G / P^{1}, R^{p-1} \pi_{*} \mathcal{L}_{\nu}\left(\mathfrak{p}^{2}\right)^{*}\right)
$$

and

$$
\left.R^{p-1} \pi_{*} \mathcal{L}_{\nu}\left(\mathfrak{p}^{2}\right)^{*}\right)=H^{p-1}\left(P^{1} / P^{2}, \mathcal{L}_{\nu}\left(\mathfrak{p}^{2}\right)_{\mid \text {fibre }}^{*}\right) .
$$

We write the decomposition in the Grothendieck group of $\mathfrak{p}^{1}$-modules:

$$
\left[H^{p-1}\left(P^{1} / P^{2}, \mathcal{L}_{\nu}\left(\mathfrak{p}^{2}\right)_{\mid \text {fibre }}^{*}\right)\right]=\sum_{\mu}{ }^{p-1} K_{P^{1}, P^{2}}^{\nu, \mu}\left[L_{\mu}\left(\mathfrak{p}^{1}\right)^{*}\right]
$$

and all the $\mu$-s are $\mathfrak{p}^{1}$-typical except $\mu=\nu$. Using the typical lemma 5 , we instantly get that

$$
\left[H^{p}\left(G / P^{1}, \mathcal{L}_{\nu}\left(\mathfrak{p}^{1}\right)^{*}\right)\right]=\sum_{\mu \neq \nu}{ }^{p-1} K_{P^{1}, P^{2}}^{\nu, \mu}\left[L_{\mu}\left(\mathfrak{p}^{1}\right)^{*}\right]
$$

the equality holding in the Grothendieck group of $\mathfrak{g}$-modules. This finishes the proof.

Now we are ready to prove Proposition 3. Since $(\lambda, \mu)$ is not an exceptional pair, the first coordinate of $\mu$ equals either the first coordinate of $\lambda$ or the second coordinate of $\lambda$. In the former case $\lambda=\mu$ (see Corollary 2). So we consider the latter case. Let $\omega$ stand for the highest weight of the standard $\mathfrak{g}$-module. By straightforward check of weights we get

$$
\begin{gathered}
\left(L_{\lambda}\left(\mathfrak{p}^{1}\right) \otimes E\right)^{\Phi^{-1}\left(\chi_{\nu}\right)}=L_{\nu}\left(\mathfrak{p}^{1}\right), \\
\left(L_{\mu} \otimes E\right)^{\chi_{\nu}}=L_{\mu+\omega} .
\end{gathered}
$$

On the other hand, if $\zeta$ is another weight such that $K_{G, P^{1}}^{\lambda, \zeta}(z) \neq 0$, we have $\left(L_{\zeta} \otimes E\right)^{\chi_{\nu}}=$ $L_{\zeta+\omega}$ if the first coordinate of $\zeta$ equals the second coordinate of $\lambda$, and $\left(L_{\zeta} \otimes E\right)^{\chi_{\nu}}=0$ otherwise. Hence if $\zeta \neq \mu,\left(L_{\zeta} \otimes E\right)^{\chi_{\nu}} \neq L_{\mu+\omega}$. Therefore, using Corollary 1 we obtain

$$
K_{G, P^{1}}^{\lambda, \mu}(z)=K_{G, P^{1}}^{\nu, \mu+\omega}(z) .
$$


By Lemma 24

$$
K_{G, P^{1}}^{\nu, \mu+\omega}(z)=z K_{P^{1}, P^{2}}^{\nu, \mu+\omega}(z) .
$$

Since $K_{P^{1}, P^{2}}^{\nu, \mu+\omega}(z)$ does not depend on the first coordinates of the weights, we have

$$
K_{P^{1}, P^{2}}^{\nu, \mu+\omega}(z)=K_{P^{1}, P^{2}}^{\nu-\omega}(z) .
$$

Note that $\nu-\omega=\lambda-\alpha$, therefore (i) of Proposition 3 is proven. To show (ii) observe that $L_{\lambda}$ occurs with multiplicity 1 in $\Gamma_{0}\left(G / P, L_{\lambda}\right)$ by Lemma 2 and does not occur in higher cohomology groups by Lemma 3. Finally, $\lambda-\alpha$ is not dominant, hence (iii) is trivial.

\section{Pretails}

We keep the assumptions of the previous section. Below we list all weights which do not satisfy the conditions of Proposition 2 or Proposition 3 ;

(1) trivial weight $\lambda=0$;

(2) the highest weight $\lambda=\varepsilon_{1}$ of the standard representation in the case $\mathfrak{g}=\mathfrak{o s p}(2 k+$ $1,2 k)$

(3) $\lambda=\varepsilon_{1}+\delta_{1}$ for $\mathfrak{g}=\mathfrak{o s p}(2 k, 2 k)$ or $\mathfrak{o s p}(2 k+2,2 k)$;

(4) $\lambda=2 \varepsilon_{1}+\delta_{1}$ for $\mathfrak{g}=\mathfrak{o s p}(2 k+1,2 k)$ or $\mathfrak{o s p}(2 k+1,2 k+2)$;

(5) $\lambda=2 \varepsilon_{1}+\varepsilon_{2}+\delta_{1}$ for $\mathfrak{g}=\mathfrak{o s p}(2 k+1,2 k)(k>2)$.

In the first case the tail of $\lambda$ has length $k$, hence $\mathfrak{q}_{\lambda}=\mathfrak{g}$. In the other four cases the tail has length $k-1$, we call such weights pretail weights. The goal of this section is to calculate $K_{G, P^{1}}^{\lambda, \mu}(z)$ for every pretail weight $\lambda$.

Let $\mathfrak{q}$ be the maximal parabolic subalgebra corresponding to the first simple root, $\mathfrak{l}$ be its Levi part. Clearly, $\mathfrak{q} \supset \mathfrak{p}^{1}$.

Lemma 25. - For any pretail weight $\lambda$ we have

$$
K_{G, P^{1}}^{\lambda, \mu}(z)=K_{G, Q}^{\lambda, \mu}(z)
$$

for all $\mu$.

Proof. - Consider the canonical projection $\pi: G / P^{1} \longrightarrow G / Q$. Note that a pretail weight $\lambda$ is $\mathfrak{p}^{1} \cap \mathfrak{l}$-typical. Hence $R^{q} \pi^{*} \mathcal{L}_{\lambda}\left(\mathfrak{p}^{1}\right)^{*}=0$ for $q>0$ and $R^{0} \pi^{*} \mathcal{L}_{\lambda}\left(\mathfrak{p}^{1}\right)^{*}=\mathcal{L}_{\lambda}(\mathfrak{q})^{*}$. Now the statement follows immediately from Leray spectral sequence.

Lemma 26. - Let $\mathfrak{g}=\mathfrak{o s p}(2 k, 2 k)$ or $\mathfrak{o s p}(2 k+2,2 k)$. Then $K_{G, Q}^{0, \mu}(z)=0$ if $\mu \neq 0$,

$$
\begin{gathered}
K_{G, Q}^{0,0}(z)=1+z^{2 k-1} \quad \text { if } \mathfrak{g}=\mathfrak{o} \mathfrak{s p}(2 k, 2 k) \\
K_{G, Q}^{0,0}(z)=1+z^{2 k} \quad \text { if } \mathfrak{g}=\mathfrak{o} \mathfrak{s p}(2 k+2,2 k) .
\end{gathered}
$$

Proof. - By Definition 1 and Penkov's remark

$$
C h\left(\Gamma_{i}(G / Q, \mathbb{C})\right) \leq C h\left(\Gamma_{i}\left(G_{0} / Q_{0}, S^{\bullet}\left(\mathfrak{g} /\left(\mathfrak{g}_{0} \oplus \mathfrak{q}_{1}\right)\right)\right)\right) .
$$

We are going to describe the simple components of the $\mathfrak{q}_{0}$-module $S^{\bullet}\left(\mathfrak{g} /\left(\mathfrak{g}_{0} \oplus \mathfrak{q}_{1}\right)\right)$.

If $\mathfrak{g}=\mathfrak{o s p}(2 k, 2 k)$, let $E^{\prime}$ denote the standard $\mathfrak{o}(2 k)$-module and $\mathfrak{q}^{\prime \prime}=\mathfrak{q} \cap \mathfrak{s p}(2 k)$, then $\mathfrak{q}_{0}=\mathfrak{o}(2 k) \oplus \mathfrak{q}^{\prime \prime}$ and one has the following isomorphism of $\mathfrak{q}_{0}$-modules

$$
S^{p}\left(\mathfrak{g} /\left(\mathfrak{g}_{0} \oplus \mathfrak{q}_{1}\right)\right) \simeq \Lambda^{p}\left(E^{\prime}\right) \otimes L_{-p \delta_{1}}\left(\mathfrak{q}^{\prime \prime}\right),
$$

where $\nabla$ means the tensor product as $\mathbb{C}$-vector spaces. If $\rho^{\prime \prime}$ is the half-sum of positive roots of $\mathfrak{s p}(2 k)$, then $-p \delta_{1}+\rho^{\prime \prime}$ is not regular for all $p$ except $p=0$ or $2 k$. The classical Borel-Weil-Bott theorem shows that there are two non-zero cohomology groups in degree 
0 and $2 k-1$. Since Lemma 2 implies that $\Gamma_{0}(G / Q, \mathbb{C}) \neq 0$, these two components can not cancel in the filtered module.

If $\mathfrak{g}=\mathfrak{o s p}(2 k+2,2 k)$, let $E^{\prime \prime}$ denote the standard $\mathfrak{s p}(2 k)$-module and $\mathfrak{q}^{\prime}=\mathfrak{q} \cap \mathfrak{o}(2 k+2)$, then $\mathfrak{q}_{0}=\mathfrak{q}^{\prime} \oplus \mathfrak{s p}(2 k)$ and one has the following isomorphism of $\mathfrak{q}_{0}$-modules

$$
S^{p}\left(\mathfrak{g} /\left(\mathfrak{g}_{0} \oplus \mathfrak{q}_{1}\right)\right) \simeq L_{-p \varepsilon_{1}}\left(\mathfrak{q}^{\prime}\right) \otimes \Lambda^{p}\left(E^{\prime \prime}\right) .
$$

Further arguments are exactly the same as in the previous case.

Lemma 27. - Let $\mathfrak{g}=\mathfrak{o s p}(2 k+1,2 k)$. Then

$$
K_{G, Q}^{\varepsilon_{1}, \mu}(z)=\left\{\begin{array}{l}
0 \text { if } \mu \neq \varepsilon_{1}, 0 \\
1 \text { if } \mu=\varepsilon_{1} \\
z^{2 k-1} \text { if } \mu=0
\end{array} .\right.
$$

Proof. - We do calculations as in the previous lemma. Let $E^{\prime \prime}$ denote the standard $\mathfrak{s p}(2 k)$ module and $\mathfrak{q}^{\prime}=\mathfrak{q} \cap \mathfrak{o}(2 k+1)$, then $\mathfrak{q}_{0}=\mathfrak{q}^{\prime} \oplus \mathfrak{s p}(2 k)$ and we have the following isomorphisms of $\mathfrak{q}_{0}$-modules

$$
\begin{aligned}
L_{\varepsilon_{1}}(\mathfrak{q}) & \simeq L_{\varepsilon_{1}}\left(\mathfrak{q}^{\prime}\right), \\
L_{\varepsilon_{1}}(\mathfrak{q}) \otimes S^{p}\left(\mathfrak{g} / \mathfrak{g}_{0} \oplus \mathfrak{q}_{1}\right) & \simeq L_{(1-p) \varepsilon_{1}}\left(\mathfrak{q}^{\prime}\right) \otimes \Lambda^{p}\left(E^{\prime \prime}\right) .
\end{aligned}
$$

There are exactly three non-acyclic components

$$
L_{\varepsilon_{1}}\left(\mathfrak{q}^{\prime}\right), E^{\prime \prime}, L_{(1-2 k) \varepsilon_{1}}\left(\mathfrak{q}^{\prime}\right) \otimes \Lambda^{2 k}\left(E^{\prime \prime}\right) .
$$

These components give rise to the standard $\mathfrak{g}$-module in degree 0 and the trivial module in degree $2 k-1$.

Proposition 4. - Let $\mathfrak{g}=\mathfrak{o s p}(2 k, 2 k)$ or $\mathfrak{o s p}(2 k+2,2 k)$ and $\lambda=\varepsilon_{1}+\delta_{1}$ be the pretail weight.

i) If $\mathfrak{g}=\mathfrak{o s p}(2 k, 2 k)$ and $k>1$, then

$$
K_{G, P^{1}}^{\lambda, \mu}(z)=\left\{\begin{array}{l}
1 \text { if } \mu=\lambda \\
1+z^{2 k-2} \text { if } \mu=0 . \\
0 \text { else }
\end{array}\right.
$$

ii) If $\mathfrak{g}=\mathfrak{o s p}(2,2)$, then

$$
K_{G, P^{1}}^{\lambda, \mu}(z)=\left\{\begin{array}{l}
1 \text { if } \mu=\lambda, 0 \\
0 \text { else }
\end{array}\right.
$$

iii) If $\mathfrak{g}=\mathfrak{o s p}(2 k+2,2 k)$ then

$$
K_{G, P^{1}}^{\lambda, \mu}(z)=\left\{\begin{array}{l}
1 \text { if } \mu=\lambda \\
z^{2 k-1} \text { if } \mu=0 . \\
0 \text { else }
\end{array}\right.
$$

Proof. - We will calculate $K_{G, Q}^{\lambda, \mu}(z)$ instead of $K_{G, P^{1}}^{\lambda, \mu}(z)$ (see Lemma 25.) Let $\omega$ be the highest weight of the standard module $E$. It is not difficult to see that $\omega$ is $\mathfrak{q}$-typical. In all cases except $\mathfrak{o s p}(2,2){ }^{3}$ there is the short exact sequence of $\mathfrak{q}$-modules:

$$
0 \rightarrow L_{\lambda}(\mathfrak{q}) \rightarrow\left(L_{\omega}(\mathfrak{q}) \otimes E\right)^{\Phi^{-1}(\chi)} \rightarrow L_{0}(\mathfrak{q})=\mathbb{C} \rightarrow 0,
$$

\footnotetext{
${ }^{3}$ for $\mathfrak{o} \mathfrak{s p}(2,2)$ this does not work since the standard $\mathfrak{o}(2)$-module is reducible and $\left(L_{\lambda}(\mathfrak{q}) \otimes E\right)^{\Phi^{-1}(\chi)}$ has one more subquotient $L_{-\varepsilon_{1}+\delta_{1}}(\mathfrak{q})$.
} 
where $\chi=\chi_{\lambda}$. As in the proof of Proposition 2, the corresponding long exact sequence degenerates in (10) and (11). It remains to study the structure of $\left(L_{\omega} \otimes E\right)^{\chi}=(E \otimes E)^{\chi}$. It is different in the two cases.

If $\mathfrak{g}=\mathfrak{o s p}(2 k+2,2 k), k>1$, then

$$
(E \otimes E)^{\chi}=L_{\varepsilon_{1}+\delta_{1}} \oplus L_{0}
$$

and Lemma 26 implies (iii).

If $\mathfrak{g}=\mathfrak{o s p}(2 k, 2 k)$, then $E \otimes E$ has two trivial subquotients. (One can see that, for instance, looking at $E \otimes E^{*}$ for $\left.\mathfrak{g l}(2 k, 2 k)\right)$. Therefore, for a suitable $V_{\lambda}$, one has the exact sequences

$$
0 \rightarrow V_{\lambda} \rightarrow(E \otimes E)^{\chi} \rightarrow L_{0} \rightarrow 0
$$

and

$$
0 \rightarrow L_{0} \rightarrow V_{\lambda} \rightarrow L_{\lambda} \rightarrow 0 .
$$

Now (i) follows from Lemma 26.

The case $\mathfrak{o s p}(2,2)$ can be easily done by a straightforward calculation similar to those in the two previous lemmas, and we leave it to the reader.

Proposition 5. - Let $\mathfrak{g}=\mathfrak{o s p}(2 k+1,2 k)$.

i) Let $\lambda_{1}=2 \varepsilon_{1}+\delta_{1}$ then

$$
K_{G, P^{1}}^{\lambda_{1}, \mu}(z)=\left\{\begin{array}{l}
1 \text { if } \mu=\lambda_{1} \text { or } \varepsilon_{1} \\
z^{2 k-2} \text { if } \mu=0 \\
0 \text { else }
\end{array} .\right.
$$

ii) If $\lambda_{2}=2 \varepsilon_{1}+\varepsilon_{2}+\delta_{1}$ then

$$
K_{G, P^{1}}^{\lambda_{2}, \mu}(z)=\left\{\begin{array}{l}
1 \text { if } \mu=\lambda_{2} \text { or } 0 \\
z^{2 k-2} \text { if } \mu=\varepsilon_{1} \\
0 \text { else }
\end{array} .\right.
$$

Proof. - Let us prove (i). As in the previous proof, using Lemma 25, we may calculate $K_{G, Q}^{\lambda, \mu}(z)$ instead of $K_{G, P^{1}}^{\lambda, \mu}(z)$. Consider the exact sequence of $\mathfrak{q}$-modules

$$
0 \rightarrow L_{\lambda_{1}}(\mathfrak{q}) \rightarrow\left(L_{\lambda_{1}-\delta_{1}}(\mathfrak{q}) \otimes E\right)^{\Phi^{-1}(\chi)} \rightarrow L_{\lambda_{1}-\alpha}(\mathfrak{q})=L_{\varepsilon_{1}}(\mathfrak{q}) \rightarrow 0 .
$$

We leave to the reader to check that all arguments in the proof of Proposition 2 go through and it holds for $\lambda=\lambda_{1}$. Thus, (i) follows from Lemma 27

To show (ii) just use the switch functor.

\section{EXCEPTIONAL PAIRS}

The goal of this section is to describe exceptional pairs. It is convenient to do in terms of weight diagrams. First, we fix some terminology. We call $<$ and $>$ core symbols. In what follows we refer to 0 (resp. $\frac{1}{2}$ ) as the tail position and denote it by $s_{0}$. For any $s<t$ in $\mathbb{T}$ we denote by $l_{f}(s, t)$ the number of $\times$-s minus the number of 0 -s strictly between $s$ and $t$. By $|f|$ we denote the double number of $\times$-s plus the number of core symbols at the tail position.

First, by the results of the previous section, if $(\lambda, \mu)$ is exceptional and $\lambda$ is a pretail, then $\mathfrak{g}=\mathfrak{o s p}(2 k+1,2 k), \lambda=2 \varepsilon_{1}+\varepsilon_{2}+\delta_{1}$ and $\mu=0$. 
Proposition 6. - Assume that $\lambda$ is not a pretail weight and let $t+1$ be the position of the rightmost $\times$ in $f_{\lambda}$. The pair $(\lambda, \mu)$ is exceptional if and only if the following conditions are true

(a) $f_{\lambda}$ is obtained from $f_{\mu}$ by moving two $\times$ from the tail position to the adjacent non-tail positions $t$ and $t+1$ (if $f_{\lambda}$ and $f_{\mu}$ have signs, they remain the same);

(b) $l_{f_{\lambda}}\left(s_{0}, t\right)$ is odd for $\mathfrak{g}=\mathfrak{o s p}(2 k+1,2 k)$ or $\mathfrak{o s p}(2 k, 2 k)$ and even for $\mathfrak{g}=\mathfrak{o s p}(2 k+2,2 k)$;

(c) $l_{f_{\lambda}}(s, t) \leq 0$ for any $s<t$;

(d) $l_{f_{\lambda}}\left(s_{0}, t\right)+\left|f_{\lambda}\right|>0$.

If $(\lambda, \mu)$ is exceptional, then $K_{G, P^{1}}^{\lambda, \mu}(z)=1$.

The proof of Proposition 6 takes the rest of the section.

\section{Lemma 28. -}

i) Let $\mathfrak{g}=\mathfrak{o s p}(2 k, 2 k), \tau=\chi_{\delta_{1}}$. If $a_{i}=1$ for some $i$, then $\left(L_{\lambda} \otimes E\right)^{\tau}=0$.

ii) Let $\mathfrak{g}=\mathfrak{o} \mathfrak{s}(2 k+2,2 k), \tau=\chi_{\varepsilon_{1}}, \kappa$ be a dominant weight with central character $\tau$. Then $\left(L_{\kappa} \otimes E\right)^{\chi}=L_{\kappa-\varepsilon_{i}} \oplus L_{\kappa+\delta_{i}}$, where $i$ is such that $a_{i}=1$.

iii) Let $\mathfrak{g}=\mathfrak{o s p}(2 k+1,2 k), \tau=\chi_{2 \varepsilon_{1}}$. If $a_{i}=\frac{3}{2}$ for some $i$, then $\left(L_{\lambda} \otimes E\right)^{\tau}=0$.

Proof. - (i) Assume first that $\lambda=\varepsilon_{1}+\delta_{1}$. If $\left(L_{\lambda} \otimes E\right)^{\tau} \neq 0$, then $\left(L_{\lambda} \otimes E\right)^{\tau}=E$ (by looking at the weights). But as follows from the calculations in the proof of Proposition 4

$$
\operatorname{Hom}_{\mathfrak{g}}\left(L_{\lambda}, E \otimes E\right)=\operatorname{Hom}_{\mathfrak{g}}\left(L_{\lambda} \otimes E, E\right)=0 .
$$

Hence $\left(L_{\lambda} \otimes E\right)^{\tau}=0$.

In the general case, by above, we have $\left(L_{\lambda}\left(\mathfrak{p}^{i-1}\right) \otimes E\right)^{\Phi^{-1}(\tau)}=0$. Hence

$$
\left(\Gamma_{0}\left(G / P^{i-1}, L_{\lambda}\left(\mathfrak{p}^{i-1}\right)\right) \otimes E\right)^{\tau}=0 .
$$

Since $L_{\lambda}$ is a quotient of $\Gamma_{0}\left(G / P^{i-1}, L_{\lambda}\left(\mathfrak{p}^{i-1}\right)\right)$ we obtain $\left(L_{\lambda} \otimes E\right)^{\tau}=0$.

(ii) We use $(E \otimes E)^{\chi}=L_{\varepsilon_{1}+\delta_{1}} \oplus L_{0}$. That implies

$$
\left(L_{\kappa}\left(\mathfrak{p}^{i-1}\right) \otimes E\right)^{\Phi^{-1}(\chi)}=L_{\kappa-\varepsilon_{i}}\left(\mathfrak{p}^{i-1}\right) \oplus L_{\kappa+\delta_{i}}\left(\mathfrak{p}^{i-1}\right) .
$$

(Keep in mind that the standard module for the Levi part of $\mathfrak{p}^{i-1}$ has highest weight $\varepsilon_{i}$ ). Therefore

$$
\left(\Gamma_{0}\left(G / P^{i-1}, L_{\kappa}\left(\mathfrak{p}^{i-1}\right)\right) \otimes E\right)^{\chi}=\Gamma_{0}\left(G / P^{i-1}, L_{\kappa-\varepsilon_{i}}\left(\mathfrak{p}^{i-1}\right)\right) \oplus \Gamma_{0}\left(G / P^{i-1}, L_{\kappa+\delta_{i}}\left(\mathfrak{p}^{i-1}\right)\right) .
$$

Clearly, $\left(L_{\kappa} \otimes E\right)^{\chi}$ is a quotient of the right hand side. Let $S$ denote the submodule of $\Gamma_{0}\left(G / P^{i-1}, L_{\kappa}\left(\mathfrak{p}^{i-1}\right)\right)$ such that $L_{\kappa}=\Gamma_{0}\left(G / P^{i-1}, L_{\kappa}\left(\mathfrak{p}^{i-1}\right)\right) / S$. All weights of $S$ are less than $\kappa$. Therefore $(S \otimes E)^{\chi}$ can not have simple components isomorphic to $L_{\kappa-\varepsilon_{i}}$ or $L_{\kappa+\delta_{i}}$, hence $\left(L_{\kappa} \otimes E\right)^{\chi}$ must have these components. Since $\left(L_{\kappa} \otimes E\right)^{\chi}$ is contragredient and its $\mathfrak{b}$ singular vectors may have only weights $\kappa-\varepsilon_{i}$ and $\kappa+\delta_{i}$, we have $\left(L_{\kappa} \otimes E\right)^{\chi}=L_{\kappa-\varepsilon_{i}} \oplus L_{\kappa+\delta_{i}}$ (see the proof of Lemma 21 for details).

(iii) First, observe that if $a_{i+1}=\frac{1}{2}$ then there are no dominant weights of the form $\lambda+\gamma$ with $\gamma$ being a weight of $E$ such that $\chi_{\lambda+\gamma}=\tau$. Hence we may assume that $a_{i+1}=-\frac{1}{2}$ or $i=k$. By comparison of weights either $\left(L_{\lambda} \otimes E\right)^{\tau}=0$ or $\left(L_{\lambda} \otimes E\right)^{\tau}=L_{\lambda-\delta_{i}}$. Consider the exact sequence as in the proof of Lemma 17

$$
0 \rightarrow S \rightarrow M_{\lambda-\beta} \rightarrow M_{\lambda} \rightarrow F \rightarrow 0,
$$

where $\beta=\varepsilon_{i}+\delta_{i}$. Check that $\left(M_{\lambda} \otimes E\right)^{\chi}$ has a filtration by Verma modules such that their highest weights are not greater than $\lambda-\delta_{i}$. Hence $L_{\lambda-\delta_{i}}$ occurs in $\left(M_{\lambda} \otimes E\right)^{\chi}$ with multiplicity 1 . Now one can finish the proof by the same arguments as in the proof of Lemma 17. 
Till the end of this section $\beta=\varepsilon_{2}+\delta_{2}, \omega$, as usual, denotes the highest weight of $E$.

Lemma 29. - Let $\mu$ and $\nu$ be dominant weights with trivial central character, $\tau=\chi_{\mu+\omega}$. Assume also that $\mu \neq 0$ in the case $\mathfrak{g}=\mathfrak{o s p}(2 k+1,2 k)$ or $\mathfrak{o s p}(2 k+2,2 k)$. If $\left(L_{\nu} \otimes E\right)^{\tau}=$ $L_{\mu+\omega}$, then $\nu=\mu$.

Proof. - For $\mu \neq \varepsilon_{1}, 0$ the lemma easily follows from Lemma 17. The condition $\mu \neq 0, \varepsilon_{1}$ ensures that $\mu+\omega=\nu+\varepsilon_{i}$ or $\mu+\omega=\nu+\delta_{i}$ for some $i$. Since $\chi_{\mu}=\chi_{\nu}=\chi_{0}$, we have $\mu=\nu$.

If $\mu=\varepsilon_{1}$, then $\mathfrak{g}=\mathfrak{o s p}(2 k+1,2 k)$ and by Lemma 28(iii) we again have $\mu+\omega=\nu+\varepsilon_{i}$ for some $i$ and the statement follows by the same reason.

Similarly, if $\mathfrak{g}=\mathfrak{o s p}(2 k, 2 k)$ and $\mu=0$, one can prove the statement using Lemma 28)(i).

Lemma 30. - Let $(\lambda, \mu)$ be an exceptional pair, $\mu_{1}=\mu+\omega$ and $\tau=\chi_{\mu+\omega}$. Assume again that $\mu \neq 0$ in the case $\mathfrak{g}=\mathfrak{o s p}(2 k+1,2 k)$ or $\mathfrak{o s p}(2 k+2,2 k)$. There exists a dominant weight $\lambda_{1}$ such that

$$
\left(L_{\lambda}\left(\mathfrak{p}^{1}\right) \otimes E\right)^{\Phi^{-1}(\tau)}=L_{\lambda_{1}}\left(\mathfrak{p}^{1}\right)
$$

and

$$
K_{G, P^{1}}^{\lambda, \mu}(z)=K_{G, P^{1}}^{\lambda_{1}, \mu_{1}}(z)=K_{G_{\tau}, P_{\tau}^{1}}^{\bar{\lambda}_{1}, \bar{\mu}_{1}}(z) .
$$

Proof. - Since $\left(L_{\mu} \otimes E\right)^{\tau}=L_{\mu_{1}}$, we know that $L_{\mu_{1}}$ occurs in some

$$
\Gamma_{i}\left(G / P^{1},\left(L_{\lambda}\left(\mathfrak{p}^{1}\right) \otimes E\right)^{\Phi^{-1}(\tau)}\right) .
$$

Hence $\left(L_{\lambda}\left(\mathfrak{p}^{1}\right) \otimes E\right)^{\Phi^{-1}(\tau)} \neq 0$. If $E^{\prime}$ denotes the standard $\mathfrak{p}^{1}$-module we have the following identity in the Grothendieck group:

$$
\begin{aligned}
& {\left[\left(L_{\lambda}\left(\mathfrak{p}^{1}\right) \otimes E\right)^{\Phi^{-1}(\tau)}\right]=\left[L_{\lambda+\varepsilon_{1}}\left(\mathfrak{p}^{1}\right)\right]+\left[L_{\lambda-\varepsilon_{1}}\left(\mathfrak{p}^{1}\right)\right]+} \\
& +\left[L_{\lambda+\delta_{1}}\left(\mathfrak{p}^{1}\right)\right]+\left[L_{\lambda-\delta_{1}}\left(\mathfrak{p}^{1}\right)\right]+\left[\left(L_{\lambda}\left(\mathfrak{p}^{1}\right) \otimes E^{\prime}\right)^{\Phi^{-1}(\tau)}\right] .
\end{aligned}
$$

Since the first coordinate of $\mu$ is less than the second coordinate of $\lambda$,

$$
\left(L_{\lambda \pm \varepsilon_{1}}\left(\mathfrak{p}^{1}\right)\right)^{\Phi^{-1}(\tau)}=\left(L_{\lambda \pm \delta_{1}}\left(\mathfrak{p}^{1}\right)\right)^{\Phi^{-1}(\tau)}=0,
$$

and

$$
\left(L_{\lambda}\left(\mathfrak{p}^{1}\right) \otimes E\right)^{\Phi^{-1}(\tau)}=\left(L_{\lambda}\left(\mathfrak{p}^{1}\right) \otimes E^{\prime}\right)^{\Phi^{-1}(\tau)} .
$$

Lemma 17 applied to $\mathfrak{p}^{1}$-modules implies that $\left(L_{\lambda}\left(\mathfrak{p}^{1}\right) \otimes E^{\prime}\right)^{\Phi^{-1}(\tau)}$ is simple, hence it is isomorphic to $L_{\lambda_{1}}\left(\mathfrak{p}^{1}\right)$ for some $\mathfrak{p}^{1}$-dominant $\lambda_{1}$. By the condition on the first coordinate of $\mu, \lambda_{1}$ is dominant.

Finally, since $\left(L_{\mu} \otimes E\right)^{\tau}=L_{\mu_{1}}$, the multiplicity of $L_{\mu_{1}}$ in $\Gamma_{i}\left(G / P^{1},\left(L_{\lambda_{1}}\left(\mathfrak{p}^{1}\right)\right)\right.$ is at least the same as the multiplicity of $L_{\mu}$ in $\Gamma_{i}\left(G / P^{1},\left(L_{\lambda}\left(\mathfrak{p}^{1}\right)\right)\right.$. Hence ${ }^{i} K_{G, P^{1}}^{\lambda, \mu} \leq{ }^{i} K_{G, P^{1}}^{\lambda_{1}, \mu_{1}}$. But by Lemma 29 $\left(L_{\nu} \otimes E\right)^{\tau} \neq L_{\mu_{1}}$ for any $\nu \neq \mu$ and we must have ${ }^{i} K_{G, P^{1}}^{\lambda, \mu}={ }^{i} K_{G, P^{1}}^{\lambda_{1}, \mu_{1}}$. Lemma 18 implies $K_{G, P^{1}}^{\lambda_{1}, \mu_{1}}(z)=K_{G_{\tau}, P_{\tau}^{1}}^{\bar{\lambda}_{1}, \bar{\mu}_{1}}(z)$.

Corollary 6. - Use the notations of the previous lemma. Let $(\lambda, \mu)$ be an exceptional pair, $r$ be the position of the rightmost $\times$ in $f_{\mu}$, and assume that $r \neq s_{0}$. Then $f_{\lambda}(r)=$ $\times, f_{\lambda}(r+1)=0,\left(\bar{\lambda}_{1}, \bar{\mu}_{1}\right)$ is an exceptional pair for $\mathfrak{g}_{\tau}$ and $K_{G, P^{1}}^{\lambda, \mu}(z)=K_{G_{\tau}, P_{\tau}^{1}}^{\bar{\lambda}_{1}, \bar{\mu}_{1}}(z)$. Furthermore, $f_{\bar{\mu}_{1}}$ is obtained form $f_{\mu}$ by removing the $\times$ from the $r$-th position and

$$
f_{\bar{\lambda}_{1}}(t)=\left\{\begin{array}{l}
f_{\lambda}(t) \text { if } t<r \\
f_{\lambda}(t+2) \text { if } t \geq r
\end{array} .\right.
$$


Lemma 31. - Let $\mathfrak{g}=\mathfrak{o} \mathfrak{s p}(2 k+2,2 k)$, and $\lambda=(2,1,0, \ldots \mid 2,1,0, \ldots)$. Then $K_{G, P^{1}}^{\lambda, 0}(z)=1$. Proof. - Let $\kappa=\lambda-\delta_{2}$ and $\tau=\chi_{\kappa}$. By Lemma 18, $K_{G, P^{1}}^{\kappa, \mu}(z)=K_{G_{\tau}, P_{\tau}^{1}}^{\bar{\kappa}, \bar{\mu}}(z)$. Therefore, by Proposition 4

By Lemma 28 (ii)

$$
K_{G, P^{1}}^{\kappa, \mu}(z)=\left\{\begin{array}{l}
1 \text { if } \mu=\kappa \\
1+z^{2 k-2} \text { if } \mu=\varepsilon_{1} \\
0 \text { else }
\end{array}\right.
$$

$$
\left(L_{\kappa}\left(\mathfrak{p}^{1}\right) \otimes E\right)^{\Phi^{-1}(\chi)}=L_{\lambda}\left(\mathfrak{p}^{1}\right) \oplus L_{\lambda-\beta}\left(\mathfrak{p}^{1}\right) .
$$

By Propositon 2 and Proposition 4 , we obtain

$$
K_{G, P^{1}}^{\lambda-\beta, \mu}(z)=\left\{\begin{array}{l}
1 \text { if } \mu=\lambda-\beta \\
z^{2 k-2} \text { if } \mu=0 \\
0 \text { else }
\end{array} .\right.
$$

By Corollary 1

$$
\left(\Gamma_{i}\left(G / P^{1}, L_{\kappa}\left(\mathfrak{p}^{1}\right)\right) \otimes E\right)^{\chi}=\Gamma_{i}\left(G / P^{1}, L_{\lambda}\left(\mathfrak{p}^{1}\right)\right) \oplus \Gamma_{i}\left(G / P^{1}, L_{\lambda-\beta}\left(\mathfrak{p}^{1}\right)\right),
$$

and by Lemma 28 (ii)

$$
(E \otimes E)^{\chi}=L_{0} \oplus L_{\varepsilon_{1}+\delta_{1}},\left(L_{\kappa} \otimes E\right)^{\chi}=L_{\lambda} \oplus L_{\lambda-\beta} .
$$

Therefore

$$
K_{G, P^{1}}^{\kappa, \varepsilon_{1}}(z)=K_{G, P^{1}}^{\lambda-\beta, 0}(z)+K_{G, P^{1}}^{\lambda, 0}(z)
$$

and we have $K_{G, P^{1}}^{\lambda, 0}(z)=1$.

Lemma 32. - Let $\mathfrak{g}=\mathfrak{o s p}(2 k+2,2 k), \tau=\chi_{\omega}, \mu$ be a dominant weight with trivial central character. One has $\left(L_{\mu} \otimes E\right)^{\tau}=E$ if and only if $\mu=0$ or $\varepsilon_{1}+\delta_{1}$.

Proof. - Since $\omega=\mu \pm \varepsilon_{i}$ or $\mu \pm \delta_{i}$, the only possible values for $\mu$ are 0 and $\varepsilon_{1}+\delta_{1}$. Obviously $\left(L_{0} \otimes E\right)^{\tau}=E$. To check that $\left(L_{\varepsilon_{1}+\delta_{1}} \otimes E\right)^{\tau}=E$, use

$$
\operatorname{Hom}_{\mathfrak{g}}\left(L_{\varepsilon_{1}+\delta_{1}} \otimes E, E\right)=\operatorname{Hom}_{\mathfrak{g}}\left(L_{\varepsilon_{1}+\delta_{1}}, E \otimes E\right)=\mathbb{C} .
$$

Lemma 33. - Let $\mathfrak{g}=\mathfrak{o s p}(2 k+2,2 k)$ (resp. osp $(2 k, 2 k))$. Then $(\lambda, 0)$ is an exceptional pair if and only if

$$
\lambda=(a, a-1,0, \ldots \mid a, a-1,0, \ldots),
$$

$a \leq 2 k-2$ is even (resp. $a \leq 2 k-3$ is odd). If $\lambda$ satisfies the above conditions, then $K_{G, P^{1}}^{\lambda, 0}(z)=1$.

Proof. - We will prove first that if $\lambda$ satisfies the conditions of Lemma, then $K_{G, P^{1}}^{\lambda, 0}(z)=1$. The proof will be done by induction on $a$ and $k$, the base case $a=2$ is done in Lemma 31. In this proof $\tau=\chi_{\omega}$.

First, let $\mathfrak{g}=\mathfrak{o s p}(2 k+2,2 k)$. Set $\lambda_{1}=\lambda+\varepsilon_{3}$. By Lemma 18 and induction assumption, we have

$$
K_{G, P^{1}}^{\lambda_{1}, \varepsilon_{1}}(z)=K_{G_{\tau}, P_{\tau}^{1}}^{\bar{\lambda}_{1}, 0}(z)=1
$$

By Lemma 28(ii) we have

$$
\left(L_{\lambda_{1}}\left(\mathfrak{p}^{1}\right) \otimes E\right)^{\Phi^{-1}(\chi)}=L_{\lambda}\left(\mathfrak{p}^{1}\right) \oplus L_{\lambda+\varepsilon_{3}+\delta_{3}}\left(\mathfrak{p}^{1}\right),
$$


and we have

$$
\operatorname{Hom}_{\mathfrak{p}^{1}}\left(L_{\lambda_{1}}\left(\mathfrak{p}^{1}\right) \otimes E, L_{\lambda}\left(\mathfrak{p}^{1}\right)\right)=\operatorname{Hom}_{\mathfrak{p}^{1}}\left(L_{\lambda_{1}}\left(\mathfrak{p}^{1}\right), L_{\lambda}\left(\mathfrak{p}^{1}\right) \otimes E\right)=\mathbb{C} .
$$

That implies

$$
\left(L_{\lambda}\left(\mathfrak{p}^{1}\right) \otimes E\right)^{\Phi^{-1}(\tau)}=L_{\lambda_{1}}\left(\mathfrak{p}^{1}\right) .
$$

Thus, by Corollary 11 and Lemma 32 we have either $K_{G, P^{1}}^{\lambda, \varepsilon_{1}+\delta_{1}}(z)=1$ or $K_{G, P^{1}}^{\lambda, 0}(z)=1$. However, the case $K_{G, P^{1}}^{\lambda, \varepsilon_{1}+\delta_{1}}(z)=1$ is impossible by Corollary 6 and we have $K_{G, P^{1}}^{\lambda, 0}(z)=1$.

Now let $\mathfrak{g}=\mathfrak{o s p}(2 k, 2 k)$. Set $\lambda_{1}=\lambda+\delta_{3}$. Using the argument similar to above we have

$$
K_{G, P^{1}}^{\lambda_{1}, \delta_{1}}(z)=1 \text {. }
$$

By Lemma 28(i), one has

$$
\left(L_{\lambda}\left(\mathfrak{p}^{1}\right) \otimes E\right)^{\Phi^{-1}(\tau)}=L_{\lambda_{1}}\left(\mathfrak{p}^{1}\right) .
$$

and by Lemma $29 K_{G, P^{1}}^{\lambda, 0}(z)=1$.

Now we will show that if $(\lambda, 0)$ is an exceptional pair, then $\lambda$ must satisfy the conditions of the lemma. The proof again is by induction on $k$. Consider two cases $f_{\lambda}(1)=\times$ and $f_{\lambda}(1)=0$.

In the first case Lemma 30 implies that $\mathfrak{g}=\mathfrak{o s p}(2 k+2,2 k)$. Let $i$ be such that $a_{i}=1$ and $\lambda_{1}=\lambda-\delta_{i} . K_{G, P^{1}}^{\lambda, 0}(z) \neq 0$ implies

$$
\left(L_{\lambda}\left(\mathfrak{p}^{1}\right) \otimes E\right)^{\Phi^{-1}(\tau)}=L_{\lambda_{1}}\left(\mathfrak{p}^{1}\right)
$$

and

By induction assumption

$$
K_{G, P^{1}}^{\lambda_{1}, \varepsilon_{1}}(z)=K_{G_{\tau}, P_{\tau}^{1}}^{\bar{\lambda}_{1}, 0}(z) \neq 0
$$

$$
\bar{\lambda}_{1}=(a, a-1,0, \ldots \mid a, a-1,0, \ldots), \lambda_{1}=(a+1, a, 1, \ldots \mid a+1, a, 0, \ldots),
$$

and $K_{G, P^{1}}^{\lambda_{1}, \varepsilon_{1}}(z)=1$. But

$$
\left(L_{\lambda_{1}}\left(\mathfrak{p}^{1}\right) \otimes E\right)^{\Phi^{-1}(\tau)}=L_{\lambda_{1}-\varepsilon_{3}}\left(\mathfrak{p}^{1}\right) \oplus L_{\lambda_{1}+\delta_{3}}\left(\mathfrak{p}^{1}\right) .
$$

Since $L_{0}$ occurs with multiplicity 1 in $\Gamma_{0}\left(G / P^{1}, L_{\lambda_{1}-\varepsilon_{3}}\left(\mathfrak{p}^{1}\right) \oplus L_{\lambda_{1}+\delta_{3}}\left(\mathfrak{p}^{1}\right)\right)$, and since we have proved already that $\left(\lambda_{1}-\varepsilon_{3}, 0\right)$ is exceptional, we obtain that $\left(\lambda_{1}+\delta_{3}, 0\right)$ is not exceptional.

Finally, if $f_{\lambda}(1)=0$, take $\lambda_{1}=\lambda+\varepsilon_{i}$ (resp. $\lambda_{1}=\lambda+\delta_{i}$ ). Repeat the above arguments to show that $\left(\bar{\lambda}_{1}, 0\right)$ is again an exceptional pair. By induction assumption

$$
\bar{\lambda}_{1}=(a, a-1,0, \ldots \mid a, a-1,0, \ldots),
$$

hence

$$
\lambda=(a+1, a, 0, \ldots \mid a+1, a, 0, \ldots) .
$$

Lemma 34. - Let $\mathfrak{g}=\mathfrak{o s p}(2 k+1,2 k)$. Then $(\lambda, 0)$ (resp. $\left.\left(\lambda, \varepsilon_{1}\right)\right)$ is an exceptional pair if and only if

(resp.

$$
\lambda+\rho=\left(a+\frac{1}{2}, a-\frac{1}{2},-\frac{1}{2}, \ldots \mid a+\frac{1}{2}, a-\frac{1}{2}, \frac{1}{2}, \ldots\right),
$$

$$
\left.\lambda+\rho=\left(a+\frac{1}{2}, a-\frac{1}{2}, \frac{1}{2},-\frac{1}{2}, \ldots \mid a+\frac{1}{2}, a-\frac{1}{2}, \frac{1}{2}, \ldots\right),\right)
$$


where $a$ is odd and $a \leq 2 k-4$. If $\lambda$ satisfies the above conditions, then $K_{G, P^{1}}^{\lambda, 0}(z)=1$ (resp. $\left.K_{G, P^{1}}^{\lambda, \varepsilon_{1}}(z)=1\right)$.

Proof. - It suffices to prove lemma for the pair $\left(\lambda, \varepsilon_{1}\right)$, since the second case follows by the use of the switch functor. The proof is similar to one for the previous lemma, but much easier. Let $\tau=\chi_{2 \varepsilon_{1}}$. Note that $K_{G, P^{1}}^{\lambda, \varepsilon_{1}}(z) \neq 0$ implies

$$
\left(L_{\lambda}\left(\mathfrak{p}^{1}\right) \otimes E\right)^{\Phi^{-1}(\tau)} \neq 0 .
$$

Then by Lemma 28 (iii) $f_{\lambda}\left(\frac{3}{2}\right)=0, f_{\lambda}$ has positive sign and

$$
\left(L_{\lambda}\left(\mathfrak{p}^{1}\right) \otimes E\right)^{\Phi^{-1}(\tau)}=L_{\lambda_{1}}\left(\mathfrak{p}^{1}\right),
$$

where $\lambda_{1}=\lambda+\varepsilon_{i}$ for $i$ such that $a_{i}=\frac{1}{2}$. Now use

$$
K_{G, P^{1}}^{\lambda, \varepsilon_{1}}(z)=K_{G, P^{1}}^{\lambda_{1}, 2 \varepsilon_{1}}(z)=K_{G_{\tau}, P_{\tau}^{1}}^{\bar{\lambda}_{1}, 0}(z)=K_{G_{\tau}, P_{\tau}^{1}}^{\bar{\lambda}_{1}^{\prime}, \varepsilon_{1}}(z),
$$

and proceed by induction decreasing $k$. The process stops when $\bar{\lambda}_{1}=2 \varepsilon_{1}+\varepsilon_{2}+\delta_{1}$ is a pretail weight (see Proposition [5).

Now Proposition 6 follows easily from Corollary [6, Lemma 33 and Lemma 34 by induction on number of $\times$ outside the tail position.

\section{Combinatorial Algorithm for calculation of $K_{G, P^{1}}^{\lambda, \mu}(z)$ And $K_{G, Q_{\lambda}}^{\lambda, \mu}(-1)$}

The recursion formulae obtained in two previous sections determine uniquely the Poincaré polynomials $K_{G, P^{1}}^{\lambda, \mu}(z)$ in the most atypical block. In this section we obtain a closed formula for them and use it to compute $K_{G, Q_{\lambda}}^{\lambda, \mu}(-1)$. The results will be formulated in terms of weight diagrams. The recursion uses double induction on the position of the rightmost $\times$ (Proposition 21) and on the total number of $\times$.

We still assume that we are in the most atypical block containing the trivial module. However, keeping track of the signs of weight diagrams in the case $\mathfrak{g}=\mathfrak{o s p}(2 k+1,2 k)$ is annoying. To get rid off those signs, we use the equivalent block of $\mathfrak{o s p}(2 k+1,2 k+2)$ with trivial central character. Weight diagrams lying in this block have $<$ at the tail position. (To obtain a signed diagram from the most atypical block of $\mathfrak{o s p}(2 k+1,2 k$ ) from such a diagram $f$, one has to shift all entries of $f$ except one at the tail position one position left and assign - if $f\left(\frac{3}{2}\right)=0$ and + if $f\left(\frac{3}{2}\right)=\times$.)

By our assumption the core symbols can only be at the tail position.

If $f(t)$ has $\times$, then let $f_{t}$ denote the diagram $g$ obtained from $f$ by removing $\times$ from position $t$, (naturally $g(t)=0$ if $t$ becomes empty). Similarly, we denote by $f^{t}$ the diagram obtained from $f$ by adding $\times$ to position $t$. Thus, $f_{s}^{t}$ denote the diagram obtained from $f$ by moving $\times$ from $s$ to $t$.

Definition 5. - We say that $g$ is obtained from $f$ by a legal move if $s<t, f(s)$ contains $\times, f(t)=0, g=f_{s}^{t}$ and one of the following conditions holds

(1) $f(s)$ does not have a core symbol, $l_{f}(s, r)>0$ for any $s<r<t$ and $l_{f}(s, t) \geq 0$;

(2) $s$ is the tail position, $|g|+l_{f}(s, r)>0$ for any $s<r<t$ and $|g|+l_{f}(s, t) \geq 0$.

The positions $s$ and $t$ are called the start and the end of a legal move $M$. The degree $l(M)$ of a legal move is defined to be $l_{f}(s, t)$ in the first case and $|g|+l_{f}(s, t)$ in the second case. A legal move is called ordinary if its start is not a tail. Otherwise we call a legal move a tail legal move. 
Warning. A legal move is actually the following data: two diagrams $f$ and $g$ and the degree of the corresponding move. Sometimes there are two legal tail moves of different degrees which transform $f$ to the same diagram $g$. They should be considered as different moves. For example, the diagram $\underset{\times}{\times}, 0, \ldots$ can be transformed to $\times, \times, \ldots$ by two different legal moves, one of degree 0 and one of degree 2 .

Definition 6. - Let $s_{0}$ denote the tail position. We say that $g$ is obtained from $f$ by an exceptional move if

$g=\left(f_{s_{0}}^{s}\right)_{s_{0}}^{t}$ for some $s<t, f(s)=f(t)=0$

$l_{f}(a, s) \leq 0$ for all $a<s$ and $|g|+l_{f}\left(s_{0}, s\right)$ is a positive odd number;

$l_{f}(s, b)>0$ for all $s<b<t$ and $l_{f}(s, t) \geq 0$.

Define the end position of an exceptional move to be $t$ and the degree to be $l_{f}(s, t)$.

Proposition 7. - Let $\lambda$ and $\mu$ be two dominant weights with trivial central character and $\lambda \neq 0$. Then ${ }^{i} K_{G, P^{1}}^{\lambda, \mu}=1$ if $\mu=\lambda$ and $i=0$ or $f_{\lambda}$ is obtained from $f_{\mu}$ by a legal move (or by an exceptional move) of degree $i$, with end at the position of the rightmost $\times$ of $f_{\lambda}$. Otherwise, ${ }^{i} K_{G, P^{1}}^{\lambda, \mu}=0$.

Proof. - If $\lambda=\mu$ the statement is obvious, if $(\lambda, \mu)$ is an exceptional pair, the statement is proven in Proposition 6. For other cases the statement follows from Propositions 2, 3, by induction on the position of the rightmost $\times$ and the number of $\times$-s in $f_{\lambda}$, the base of induction being done in Propositions 4, 5 and Lemma 27. One has to translate Proposition 4 and Lemma 27 from signed diagrams to diagrams with $<$ in the tail position, which can be done by direct comparison.

Corollary 7. - The multiplicity of any simple module in $\Gamma_{i}\left(G / P^{1}, L_{\lambda}\left(\mathfrak{p}^{1}\right)\right)$ is at most one.

Corollary 8. - Let $\lambda$ and $\mu$ be two dominant weights with trivial central character and $\lambda \neq 0$. Let $t$ be the position of $j$-th $\times$ in $f_{\lambda}$ counting from the right. Then ${ }^{i} K_{P^{j-1}, P^{j}}^{\lambda, \mu}=1$ if $\mu=\lambda$ and $i=0$, or if $f_{\lambda}$ is obtained from $f_{\mu}$ by a legal move (or by an exceptional move) of degree $i$ with end $t$. Otherwise, ${ }^{i} K_{P^{j-1}, P^{j}}^{\lambda, \mu}=0$.

Let $D_{\mathfrak{g}}$ denote the oriented graph whose vertices are dominant weights of $\mathfrak{g}$, and edges are defined as follows:

if $f_{\lambda}$ is obtained from $f_{\mu}$ by a legal move or exceptional move, we join $\lambda$ and $\mu$ by an edge $\mu \longrightarrow \lambda$.

We put a label $(s, t ; w)$ on an edge, where $s$ and $t$ are the start and the end of the corresponding legal move and $w$ is its degree. If the move is exceptional we put the label $\left(s_{0}: s, t ; w\right)$.

A path consisting of edges corresponding to legal moves with ends $t_{1}, \ldots, t_{q}$ is called decreasing resp. increasing if $t_{1}>\ldots>t_{q}$ (resp. $t_{1}<\ldots<t_{q}$ ). (It follows immediately from the definition that, in any path, $t_{i} \neq t_{i+1}$.) The degree $l(R)$ of a path $R$ is the sum of the degrees of all legal moves corresponding to the edges included in $R$. It is straightforward that $D_{\mathfrak{g}}$ does not have oriented cycles.

Theorem 3. - Let $\mathcal{P}^{>}(\mu, \lambda)$ denote the set of all decreasing paths from $\mu$ to $\lambda$. Then

$$
K_{G, Q_{\lambda}}^{\lambda, \mu}(-1)=\sum_{R \in \mathcal{P}>(\mu, \lambda)}(-1)^{l(R)} .
$$


Proof. - Let $r$ be the number of $\times$ outside the tail position in $f_{\lambda}$. Then Theorem 1 and Corollary 8 imply

(14)

$K_{G, Q_{\lambda}}^{\lambda, \mu}(-1)=\sum_{\mu \leq \mu_{1} \leq \ldots \leq \mu_{r} \leq \lambda} K_{P^{r-1}, P^{r}}^{\lambda, \mu_{r}}(-1) K_{P^{r-1}, P^{r-2}}^{\mu_{r}, \mu_{r-1}}(-1) \ldots K_{G, P^{1}}^{\mu_{1}, \mu}(-1)=\sum_{R \in \mathcal{P}>(\mu, \lambda)}(-1)^{l(R)}$.

Remark - One can easily generalize Theorem 3 to an arbitrary block due to Corollary 5 . Legal and exceptional moves are described in the same way. We completely ignore core symbols outside the tail position.

\section{Characters}

In this section we give a combinatorial algorithm for computing characters of simple modules.

Let $\mathcal{E}_{\lambda}$ denote the right hand side of formula (2) in Proposition 1 with $\mathfrak{p}=\mathfrak{q}_{\lambda}$. Note that since $L_{\lambda}\left(\mathfrak{q}_{\lambda}\right)$ is one-dimensional, $C h\left(L_{\lambda}\left(\mathfrak{q}_{\lambda}\right)\right)=e^{\lambda}$.

The identity (2) provides a linear system of equations, which can be solved for $C h\left(L_{\lambda}\right)$. Let $\mathbb{K}$ denote the infinite matrix with coefficients $K_{G, Q_{\lambda}}^{\lambda, \mu}(-1)$. Then $\mathbb{K}$ is lower triangular with 1 on diagonal. Let $\mathbb{D}=\mathbb{K}^{-1}$, and $D^{\lambda, \mu}$ denote the matrix coefficients of $\mathbb{D}$. Then (2) implies

$$
C h\left(L_{\lambda}\right)=\sum D^{\lambda, \mu} \mathcal{E}_{\mu}
$$

Remark - One can see that the graphs $D_{\mathfrak{g}}$ and the matrices $\mathbb{K}$ and $\mathbb{D}$ for $\mathfrak{g}=\mathfrak{o s p}(2 k+2,2 k)$ and $\mathfrak{g}=\mathfrak{o s p}(2 k+1,2 k)$ are the same if one identifies weight diagrams by switching $>$ to $<$ at the tail position. It is natural to conjecture that the maximal atypical blocks in these two cases are equivalent.

Theorem 4. - Let $\mathcal{P}^{<}(\mu, \lambda)$ denote the set of increasing paths from $\mu$ to $\lambda$ in $D_{\mathfrak{g}}$, and $|R|$ denote the number of edges in a path $R$. Then

$$
D^{\lambda, \mu}=\sum_{R \in \mathcal{P}<(\mu, \lambda)}(-1)^{l(R)+|R|} .
$$

Proof. - Write $\mathbb{K}=1+\mathbb{U}$, where $\mathbb{U}$ is strictly low triangular. Then

$$
\mathbb{D}=1-\mathbb{U}+\mathbb{U}^{2}-\ldots
$$

Let $R_{1} \circ R_{2}$ denote the concatenation of paths $R_{1}$ and $R_{2}$. Then (13) implies

$$
D^{\lambda, \mu}=\sum_{R \in \mathcal{P}(\mu, \lambda)} \sum_{R=R_{1} \circ R_{2} \ldots \circ R_{i} \mid R_{j} \in \mathcal{P}>}(-1)^{i+l(R)},
$$

where $\mathcal{P}(\mu, \lambda)$ denotes the set of all paths from $\mu$ to $\lambda$ and $\mathcal{P}^{>}$is the set of all decreasing paths.

Any path $R \notin \mathcal{P}^{<}(\mu, \lambda)$ has more than one term in the second sum, since there are several ways to write it as a concatenation of decreasing paths. It is a simple exercise to check that in this case the second sum is zero. Hence the only paths contributing to the formula are increasing. Hence (16) holds. 
Remark - In case $\mathfrak{g}=\mathfrak{o s p}(2 k, 2 k)$ Theorem 4 provides the formula for $C h\left(L_{\lambda}\right)$ only for positive $\lambda$. For negative $\lambda$, we apply the automorphism $\sigma$ defined in Section 7, and we use

$$
D^{\lambda, \mu}=D^{\lambda^{\prime}, \mu^{\prime}} .
$$

Example. Let $\mathfrak{g}=\mathfrak{o s p}(6,6)$ and $\lambda=(2,1,0 \mid 2,1,0)$. To find $C h\left(L_{\lambda}\right)$, we just have to describe the subgraph $D_{\mathfrak{g}}$ containing the vertices $\mu \leq \lambda$. There are four such vertices corresponding to the weights $\lambda, \mu=(2,0,0 \mid 2,0,0), \nu=(1,0,0 \mid 1,0,0), \kappa=0$. They are connected by the edges:

$$
\begin{aligned}
& \kappa \stackrel{(0,1 ; 0)}{\longrightarrow} \nu, \\
& \kappa \stackrel{(0,1 ; 4)}{\longrightarrow} \nu, \\
& \kappa \stackrel{(0,2 ; 3)}{\longrightarrow} \mu, \\
& \nu \stackrel{(1,2 ; 0)}{\longrightarrow} \mu, \\
& \nu \stackrel{(0,2 ; 1)}{\longrightarrow} \lambda, \\
& \nu \stackrel{(0,2 ; 1)}{\longrightarrow} \lambda, \\
& \mu \stackrel{(0,1 ; 0)}{\longrightarrow} \lambda, \\
& \mu \stackrel{(0,1 ; 2)}{\longrightarrow} \lambda .
\end{aligned}
$$

Then the corresponding matrices are

$$
\begin{aligned}
\mathbb{K} & =\left(\begin{array}{cccc}
1 & 0 & 0 & 0 \\
2 & 1 & 0 & 0 \\
0 & 1 & 1 & 0 \\
-2 & -1 & 2 & 1
\end{array}\right) \\
\mathbb{D} & =\left(\begin{array}{cccc}
1 & 0 & 0 & 0 \\
-2 & 1 & 0 & 0 \\
2 & -1 & 1 & 0 \\
-4 & 3 & -2 & 1
\end{array}\right)
\end{aligned}
$$

and

$$
C h\left(L_{\lambda}\right)=\mathcal{E}_{\lambda}-2 \mathcal{E}_{\mu}+2 \mathcal{E}_{\nu}-4 \mathcal{E}_{\kappa} .
$$

The case $k=1$. (See [10, 13]) If $\mathfrak{g}=\mathfrak{o s p}(2,2)$, positive weights in the most atypical block are of the form $a \varepsilon_{1}+a \delta_{1}$. To simplify notations we put $L_{a}=L_{a \varepsilon_{1}+a \delta_{1}}$. The graph $D_{\mathfrak{g}}$ is the infinite string

$$
0 \stackrel{(0,1 ; 0)}{\longrightarrow} 1 \stackrel{(1,2 ; 0)}{\longrightarrow} 2 \stackrel{(2,3 ; 0)}{\longrightarrow} \ldots,
$$

and the characters can be calculated by

$$
C h\left(L_{a}\right)=\sum_{j=0}^{a}(-1)^{a+j} \mathcal{E}_{j} .
$$

The matrix $\mathbb{D}$ is the same for $\mathfrak{o s p}(4,2)$ and $\mathfrak{o s p}(3,2)$. Let us consider the latter case. Set $\lambda_{0}=0, \lambda_{1}=\varepsilon_{1}, \lambda_{i}=i \varepsilon_{1}+(i-1) \delta_{1}$. The graph $D_{\mathfrak{g}}$ has the edges $\lambda_{i} \stackrel{(i, i+1 ; 0)}{\longrightarrow} \lambda_{i+1}$ for 
all $i \geq 1$ and the edges $\lambda_{0} \stackrel{(0,2 ; 0)}{\longrightarrow} \lambda_{2}, \lambda_{0} \stackrel{(0,1 ; 1)}{\longrightarrow} \lambda_{1}$. One can easily obtain the character formulae

$$
C h\left(L_{\lambda_{p}}\right)=\sum_{i=1}^{p}(-1)^{p+i} \mathcal{E}_{\lambda_{i}}-2(-1)^{p} \mathcal{E}_{\lambda_{0}}=\sum_{i=1}^{p}(-1)^{p+i} \mathcal{E}_{\lambda_{i}}-2(-1)^{p},
$$

for $p \geq 2$, and

$$
C h\left(L_{\lambda_{1}}\right)=\mathcal{E}_{\lambda_{1}}+\mathcal{E}_{\lambda_{0}}=\mathcal{E}_{\lambda_{1}}+1
$$

\section{Caps and CANCEllations}

Let $f$ be a weight diagram. For every $\times$ at a non-tail position $s$, there exists exactly one legal move $f \rightarrow f^{\prime}$ of degree zero with start at $s$. If $t$ is the end of that move, then we join $s$ and $t$ by a cap. Proceeding in this way we equip $f$ with caps for each non-tail $s$ such that $f(s)=\times$. A non-tail position $s$ is called free if $f(s)=0$ and $s$ is not an end of a cap. One can easily check the following properties:

- there are no free positions under a cap;

- two caps do not overlap;

- if $s \neq s_{0}, f(s)=\times$, then the end of a legal move with start at $s$ is not larger than the end of the cap starting at $s$.

We call a decreasing path $R \in \mathcal{P}^{>}(\mu, \lambda)$ regular if

- any edge of $R$ corresponding to a non-tail legal move corresponds to a move along a cap on the diagram $f_{\mu}$;

- any edge of $R$ corresponding to a tail legal move or an exceptional move corresponds to a move with end at a free position.

Note that all ordinary and exceptional moves which appear in a regular path must have degree 0. In addition, it follows directly from Definition 6 that the position $s$ in any exceptional legal move $\left(s_{0}: s, t ; w\right)$ which appears in a decreasing path from $\mu$ to $\lambda$ is a free position of $f_{\mu}$.

Proposition 8. - Let $\mathcal{R} \mathcal{P}^{>}(\mu, \lambda)$ denote the set of all regular decreasing paths from $\mu$ to $\lambda$. Then

$$
\sum_{R \in \mathcal{P}>(\mu, \lambda)}(-1)^{l(R)}=\sum_{R \in \mathcal{R} \mathcal{P}^{>}(\mu, \lambda)}(-1)^{l(R)} .
$$

Proof. - Define an involution $*$ on the set of all non-regular paths in $\mathcal{P}^{>}(\mu, \lambda)$ as follows. If $R$ is a non-regular path, one can find at least one cap whose left or right end is the end of some "wrong" legal move included in $R$ which is not a move along this cap. Among such caps, pick up a cap with maximal left end. There are two possibilities:

1. The left end $t$ of this cap is the end of some "wrong" legal move $(s, t ; w)$ (resp. "wrong" exceptional move $\left.\left(s_{0}: s, t ; w\right)\right)$. Then, before the edge $(s, t ; w)\left(\operatorname{resp} .\left(s_{0}: s, t ; w\right)\right)$ in the path $R$, there is an edge $(t, u ; 0)$ which, by our conditions, corresponds to a legal move along a cap. Exchange two edges $(s, t ; w)$ (resp. $\left.\left(s_{0}: s, t ; w\right)\right)$ and $(t, u ; 0)$ by one which corresponds to a legal move $(s, u ; w+1)$ (resp. exceptional move $\left(s_{0}: s, u ; w+1\right)$ ) and get a new irregular path $R^{*}$. One can easily check that $l\left(R^{*}\right)=l(R)+1$.

2. The right end $t$ of the cap is the end of some "wrong" legal move $(s, t ; w)$ (resp. "wrong" exceptional move $\left.\left(s_{0}: s, t ; w\right)\right)$. Note that in this case $w$ must be positive. Let $u$ be the left end of the cap. Remove the edge $(s, t ; w)$ (resp. $\left.\left(s_{0}: s, t ; w\right)\right)$ and insert $(u, t ; 0)$ and $(s, u ; w-1)$ (resp. $\left.\left(s_{0}: s, u ; w-1\right)\right)$ and get a new irregular path $R^{*}$. One can easily check that $l\left(R^{*}\right)=l(R)-1$. 
Obviously, $*$ is an involution. The statement follows from the condition $(-1)^{l\left(R^{*}\right)}=$ $-(-1)^{l(R)}$.

Proposition 9. - If $\mathfrak{g}=\mathfrak{o s p}(2 n+1,2 n)$ or $\mathfrak{o s p}(2 n+2,2 n)$ and $\lambda, \mu$ have the trivial central character, then $K_{G, Q_{\lambda}}^{\lambda, \mu}(-1)$ is either zero or \pm 1 . In other words, the entries of $\mathbb{K}$ are 0 or \pm 1 .

If $\mathfrak{g}=\mathfrak{o s p}(2 n, 2 n)$ and $\lambda, \mu$ have trivial central character, then $\left|K_{G, Q_{\lambda}}^{\lambda, \mu}(-1)\right| \leq 2$

Proof. - By Proposition 8 and Theorem 3 we have

$$
K_{G, Q_{\lambda}}^{\lambda, \mu}(-1)=\sum_{R \in \mathcal{R} \mathcal{P}^{>}(\mu, \lambda)}(-1)^{l(R)} .
$$

Let $t_{1}, t_{2}, \ldots$ be all free positions written in increasing order. Let $R$ be some decreasing regular path. We call two tail moves appearing in $R$ adjacent if they have ends $t_{i}$ and $t_{i-1}$. A pair of adjacent tail moves is vanishing if there exists an exceptional move with label $\left(s_{0}: t_{i-1}, t_{i} ; 0\right)$. (In particular, $i$ is odd in case $\mathfrak{g}=\mathfrak{o s p}(2 n+1,2 n)$ or $\mathfrak{o s p}(2 n+2,2 n)$ and even in case $\mathfrak{g}=\mathfrak{o s p}(2 n, 2 n)$.)

If $R$ is a regular but not strongly regular decreasing path, we pick up the first exceptional move or the first vanishing adjacent pair which appears in it, depending on what occurs earlier. Denote by $R^{\prime}$ the path obtained from $R$ by substituting the vanishing pair instead of the first exceptional move (or respectively the exceptional move instead of the first vanishing pair). Then clearly $R^{\prime \prime}=R$ and $R$ and $R^{\prime}$ cancell in the summation of (18). Hence the sum can be taken only over strongly regular paths.

In case $\mathfrak{g}=\mathfrak{o} \mathfrak{s p}(2 n+1,2 n)$ or $\mathfrak{o} \mathfrak{s p}(2 n+2,2 n)$ one can see immediately that there is at most one strongly regular path between any two weight diagrams.

If $\mathfrak{g}=\mathfrak{o s p}(2 n, 2 n)$ there are two tail moves with the end $t_{1}$, one of degree 0 and one of degree equal to the double size of the tail. Hence there are at most two strongly regular paths between two weight diagrams. Hence the statement.

\section{Appendix: INDEX OF DEFINITIONS AND NOTATIONS}

\section{General Setting}

- Integral dominant weight: Section 2.

- $\Phi$ : just before Lemma 4 .

- $A(\lambda)$ : Definition 2 .

- Degree of atypicality of a dominant weight (resp. central character): Definition 2 (resp. just after this definition).

- Admissible parabolic subalgebra for a central character: Definition 3.

- Poincaré polynomial $K_{A, B}^{\lambda, \mu}(z)$ : Definition 4 .

- List of simple roots for the chosen Borel subalgebras: Section 5.

- Dominance contitions for weights: Section 5.

- Core of a central character: just before Lemma 7 .

- $\mathfrak{g}_{\chi}$ (for a central character $\left.\chi\right)$ : before Lemma 8 .

- Stable weights: before Lemma 8 . 
- $\mathcal{F}_{\leq \lambda}^{\chi}$ : before Lemma 8 ,

- Translation functors $T(V)^{\chi, \tau}$ : after the proof of Lemma 8 .

- E: Standard $\mathfrak{g}$-module.

- Case $\mathfrak{o s p}(2 m, 2 n)$, positive (resp. negative) weights: Section 6 .

- Tail subalgebra of a dominant weight $\lambda, \mathfrak{g}_{\lambda}$; tail of $\lambda$; algebra $\mathfrak{q}_{\lambda}$ : just before Lemma 15.

- Switch functor $T(E)^{\chi, \chi}$ : after the proof of Lemma 19 ,

- Exceptional pair of weights: before Proposition 3 ,

- Pretail weights: Section 9.

\section{WEIGHT DIAGRAMS AND ALGORITHM}

- Diagram $f_{\lambda}$ associated to a weight $\lambda$ : defined in the beginning of Section 6 . We will use the notations introduced there for this part of the appendix.

- Diagram of the core: the diagram one obtains from $f_{\lambda}$ when removing all the symbols $\times$.

- Is it possible to recover the weight from the diagram? Yes if $\mathfrak{g}=\mathfrak{g l}(m, n)$, no if $\mathfrak{g}=$ $\mathfrak{o s p}(m, 2 n)$ (see Section 6).

- Number of $\times$-s in the diagram: atypicality degree of the corresponding weight.

- Indicator \pm and sign: see $\mathfrak{o s p}(2 n+1,2 n)$ in Section 6 (depends on the tail's shape).

- Action of translation functor: see section 6 .

- Relationship between the number of $\times$-s at 0 and the length of the tail of $\lambda$ : just before Lemma 15.

- Core symbols: beginning of Section 10.

- Notation $s_{0}$ : tail position.

- Notation $l_{f}(s, t)$ : number of $\times-\mathrm{s}-$ number of 0 -s strictly between the positions $s$ and $t$.

- Notation $|f|: 2 \times$ number of $\times$-s at the tail position + number of core symbols at the tail position.

- Notation $f_{t}$ : If $f$ has a $\times$ at position $t$, the $f_{t}$ is the diagram obtained when removing it.

- Notation $f^{t}$ : Diagram obtained adding to $f$ a $\times$ at position $t$.

- Notation $f_{s}^{t}$ : diagram obtained from $f$ moving a $\times$ from $s$ to $t$.

- Legal moves, start and end of a legal move, degree of a legal move: Definition 5, Section 11.

- Tail legal move, ordinary legal move: Definition 5, Section 11.

- Exceptional move: Definition 6, Section 11.

- Decreasing, increasing paths in the oriented graph $D_{\mathfrak{g}}$ : just after Corollary 8 . 
- Length of a path in a $D_{\mathfrak{g}}$ : just before Theorem 3 ,

- Cap: Section 13.

- Free position: Section 13.

- Strongly regular: Section 13.

- Regular path: Section 13.

\section{REFERENCES}

[1] J. Bernstein, S. Gelfand, Tensor products of finite and infinite-dimensional representations of semisimple Lie algebras, Compositio Math. 41 (1980), 245-285.

[2] J. Bernstein, D. Leites, A formula for the characters of the irreducible finite-dimensional representations of Lie superalgebras of series Gl and sl. (Russian) C. R. Acad. Bulgare Sci. 33 (1980), no. 8, 1049-1051.

[3] J. Brundan, Kazhdan-Lusztig polynomials and character formulae for the Lie superalgebra $g l(m \mid n)$. J. Amer. Math. Soc. 16 (2003), no. 1, 185-231.

[4] J. Brundan, C. Stroppel, Highest weight categories arising from Khovanov's diagram algebra I: cellularity. Preprint, 2008.

[5] J. Brundan, C. Stroppel, Highest weight categories arising from Khovanov's diagram algebra II: Koszulityty. Preprint, 2008.

[6] J. Brundan, C. Stroppel, Highest weight categories arising from Khovanov's diagram algebra III: Category $\mathcal{O}$. Preprint, 2008.

[7] S. J. Cheng, N. Lam, Irreducible characters of general linear superalgebra and super duality. arXiv:0905.0332 1 .

[8] S. J. Cheng, W. Wang, Brundan-Kazhdan-Lusztig and superduality conjecture. Publ. RIMS, Kyoto Univ. 44 (2008), 1219-1272.

[9] M. Duflo, V. Serganova, On associated variety for Lie superalgebras, math/0507198

[10] J. Germoni, Indecomposable representations of $\operatorname{osp}(3,2), D(2,1 ; \alpha)$ and $G(3)$. Colloquium on Homology and Representation Theory (Spanish) . Bol. Acad. Nac. Cienc. (Cordoba) 65 (2000), 147-163.

[11] M. Gorelik, Annihilation theorem and separation theorem for basic classical Lie superalgebras. J. Amer. Math. Soc. 15 (2002), no. 1, 113-165

[12] C. Gruson, Sur l'ideal du cone autocommutant des super algebres de Lie basiques classiques et etranges. (French) [On the ideal of the self-commuting cone of basic classical and strange Lie superalgebras], Ann. Inst. Fourier (Grenoble), 50 (2000), no. 3. 807-831.

[13] C. Gruson, Cohomologie des modules de dimension finie sur la super algebre de Lie osp (3, 2). (French) [Cohomology of finite-dimensional modules over the Lie superalgebra osp (3,2)] J. Algebra 259 (2003), no. 2, 581-598.

[14] C. Gruson, S. Leidwanger, Cones nilpotents des super algebres de Lie orthosymplectiques (preprint available on Severine Leidwanger's web page)

[15] J. Jantzen, Representations of algebraic groups. Second edition. Mathematical Surveys and Monographs, 107. American Mathematical Society, Providence, RI, 2003.

[16] V. Kac, Lie superalgebras. Advances in Math. 26 (1977), no. 1, 8-96.

[17] V. Kac, Characters of typical representations of classical Lie superalgebras. Comm. Algebra 5 (1977), no. 8, 889-897.

[18] V. Kac, M. Wakimoto, Integrable highest weight modules over affine superalgebras and number theory. Lie theory and geometry, 415-456, Progr. Math., 123, Birkhauser, Boston, MA, 1994.

[19] Yu. Manin, Gauge field theory and complex geometry. Translated from the 1984 Russian original by N. Koblitz and J. R. King. Grundlehren der Mathematischen Wissenschaften [Fundamental Principles of Mathematical Sciences], 289. Springer-Verlag, Berlin, 1997.

[20] Yu. Manin, I. Penkov, A. Voronov, Elements of supergeometry. (Russian) Translated in J. Soviet Math. 51 (1990), no. 1, 2069-2083.

[21] Yu. Manin, A. Voronov, Supercellular partitions of flag superspaces. (Russian) Translated in J. Soviet Math. 51 (1990), no. 1, 2083-2108. 
[22] I. Musson, V. Serganova Combinatorics of character formulas for the Lie superalgebra $\mathfrak{g l}(m, n)$, Preprint.

[23] I. Penkov, Borel-Weil-Bott theory for classical Lie supergroups. (Russian) Translated in J. Soviet Math. 51 (1990), no. 1, 2108-2140.

[24] V. Serganova, Kazhdan-Lusztig polynomials and character formula for the Lie superalgebra $g l(m \mid n)$. Selecta Math. (N.S.) 2 (1996), no. 4, 607-651.

[25] V. Serganova, Characters of irreducible representations of simple Lie superalgebras. Proceedings of the International Congress of Mathematicians, Vol. II (Berlin, 1998).

[26] V. Serganova, A reduction method for atypical representations of classical Lie superalgebras. Adv. Math. 180 (2003), no. 1, 248-274.

[27] V. Serganova, Kac-Moody superalgebras and integrability. To appear in "Perspectives of infinitedimensional Lie theorey", Birkhauser.

[28] A. Sergeev, The invariant polynomials on simple Lie superalgebras. Represent. Theory 3 (1999), 250280.

[29] J. Van der Jeugt, Character formulae for Lie superalgebra $C(n)$, Comm. Algebra 19, (1991), no. 1, 199-222.

[30] J. Van der Jeugt, J. W. B. Hughes, R. C. King, J. Thierry-Mieg, A character formula for singly atypical modules of Lie superalgebra $s l(m, n)$, Comm. Algebra 18, (1990), no. 10, 3453-3480. 